\title{
OUT OF EQUILIBRIUM FIELDS IN INFLATIONARY DYNAMICS. DENSITY FLUCTUATIONS
}

\author{
D. Boyanovsky ${ }^{(a)}$, D. Cormier ${ }^{(b)}$, H. J. de Vega ${ }^{(c)}$, R. Holman ${ }^{(b)}$, S. P. Kumar ${ }^{(b)}$ \\ (a) Department of Physics and Astronomy, University of Pittsburgh, Pittsburgh, PA. 15260, \\ U.S.A. \\ (b) Department of Physics, Carnegie Mellon University, Pittsburgh, PA. 15213, U. S. A. \\ (c) LPTHE, Université Pierre et Marie Curie (Paris VI) et Denis Diderot (Paris VII), Tour 16, \\ 1er. étage, 4, Place Jussieu 75252 Paris, Cedex 05, France
}

(December 1997)

\begin{abstract}
The energy and time scales during the inflationary stage of the universe calls for an out of equilibrium quantum field treatment. Moreover, the high energy densities involved $\left(\sim 1 / g \sim 10^{12}\right)$ make necessary the use of non-perturbative approaches as the large $N$ and Hartree methods. We start these lectures by introducing the such non-perturbative out of equilibrium methods in cosmological universes. We discuss the renormalization procedure and the choice of initial conditions. We then study with these methods the non-linear dynamics of quantum fields in matter and radiation dominated FRW and de Sitter universes. For a variety of initial conditions, we compute the evolution of the inflaton, its quantum fluctuations and the equation of state. We investigate the phenomenon of explosive particle production due to spinodal instabilities and parametric amplification in FRW and de Sitter universes with and without symmetry breaking. We find that the particle production is somewhat sensitive to the expansion of the universe. In the large $N$ limit for symmetry breaking scenarios, we determine generic late time fields behavior for any flat FRW and de Sitter cosmology. We find that quantum fluctuations damp in FRW as the square of the scale factor while the order parameter approaches a minimum of the potential at the same rate. We present a complete and numerically accessible renormalization scheme for the equation of motion and the energy momentum tensor in flat cosmologies. In this scheme the renormalization constants are independent of time and of the initial conditions. Furthermore, we consider an $O(N)$ inflaton model coupled self-consistently to gravity in the semiclassical approximation, where the field is subject to 'new inflation' type initial conditions. We study the dynamics self-consistently and non-perturbatively with non-equilibrium field theory methods in the large $N$ limit. We find that spinodal instabilities drive the growth of non-perturbatively large quantum fluctuations which shut off the inflationary growth of the scale factor. We find that a very specific combination of these large fluctuations plus the inflaton zero mode assemble into a new effective field. This new field behaves classically and it is the object
\end{abstract}


which actually rolls down. We show how this reinterpretation qualitatively saves the standard picture of how metric perturbations are generated during inflation and that the spinodal growth of fluctuations dominates the time dependence of the Bardeen variable for superhorizon modes during inflation. We compute the amplitude and index for the spectrum of scalar density and tensor perturbations and argue that in all models of this type the spinodal instabilities are responsible for a 'red' spectrum of primordial scalar density perturbations. The decoherence aspects and the quantum to classical transition through inflation are studied in detail by following the full evolution of the density matrix.

\section{INTRODUCTION AND MOTIVATION}

Inflationary cosmology has come of age. From its beginnings as a solution to deep problems such as the horizon, flatness, entropy and monopole problems [1], it has grown into the main contender for the source of primordial fluctuations giving rise to large scale structure [33]. There is evidence from the measurements of temperature anisotropies in the cosmic microwave background radiation (CMBR) that the scale invariant power spectrum predicted by generic inflationary models is at least consistent with observations [2 [4] and we can expect further and more exacting tests of the inflationary power spectrum when the MAP and PLANCK missions are flown. In particular, if the fluctuations that are responsible for the temperature anisotropies of the CMB truly originate from quantum fluctuations during inflation, determinations of the spectrum of scalar and tensor perturbations will constrain inflationary models based on particle physics scenarios and probably will validate or rule out specific proposals [3, 34].

The tasks for inflationary universe researchers are then two-fold. First, models of inflation must be constructed on the basis of a realistic particle physics model. This is in contrast to the current situation where most, if not all acceptable inflationary models are ad-hoc in nature, with fields and potentials put in for the sole purpose of generating an inflationary epoch. Second, and equally important, we must be sure that the quantum dynamics of inflation is well understood. This is extremely important, especially in light of the fact that it is exactly this quantum behavior that is supposed to give rise to the primordial metric perturbations which presumably have imprinted themselves in the CMBR. This latter problem is the focus of this review.

The inflaton must be treated as a non-equilibrium quantum field. The simplest way to see this comes from the requirement of having small enough metric perturbation amplitudes which in turn requires that the quartic self coupling $\lambda$ of the inflaton be extremely small, typically of order $\sim 10^{-12}$. Such a small coupling cannot establish local thermodynamic equilibrium (LTE) for all field modes; typically the long wavelength modes will respond too slowly to be able to enter LTE. In fact, the superhorizon sized modes will be out of the region of causal contact and cannot thermalize. We see then that if we want to gain a deeper understanding of inflation, non-equilibrium tools must be developed. Such tools exist and have now been developed to the point that they can give quantitative answers to these questions in cosmology [9] - [20], [26,27,39,40]. These methods permit us to follow the dynamics of quantum fields in situations where the energy density is non-perturbatively 
large $(\sim 1 / \lambda)$. That is, they allow the computation of the time evolution of non-stationary states and of non-thermal density matrices.

Our programme on non-equilibrium dynamics of quantum field theory, started in 1992 [9], is naturally poised to provide a framework to study these problems. The larger goal of the program is to study the dynamics of non-equilibrium processes from a fundamental field-theoretical description, by solving the dynamical equations of motion of the underlying four dimensional quantum field theory for physically relevant problems: the early universe dynamics, high energy particle collisions, phase transitions out of equilibrium, symmetry breaking and dissipative processes.

The focus of our work is to describe the quantum field dynamics when the energy density is high. That is, a large number of particles per volume $m^{-3}$, where $m$ is the typical mass scale in the theory. Usual S-matrix calculations apply in the opposite limit of low energy density and since they only provide information on in $\rightarrow$ out matrix elements, are unsuitable for calculations of expectation values.

In high energy density situations such as in the early universe, the particle propagator (or Green function) depends on the particle distribution in momenta in a nontrivial way. This makes the quantum dynamics intrinsically nonlinear and calls to the use of self-consistent non-perturbative approaches as the large $N$ limit, Hartree and self-consistent one-loop approximations.

There are basically three different levels to study the early universe dynamics:

1. To work out the nonlinear dynamics of quantum fields in Minkowski spacetime. By non-linear dynamics we understand to solve the quantum equations of motion including the quantum back-reaction quantitatively [9] - [12], [24, 31, 39, 40]. This level is in fact appropriate to describe high energy particle collisions 42 .

2. To work out the nonlinear dynamics of quantum fields in fixed cosmological backgrounds 14,15]. New phenomena arise then compared with 1 . showing that a Minkowski analysis is not quantitatively precise for expanding universes.

3. A self-consistent treatment of the quantum fields and the cosmological background [16,41]. That is, the metric is obtained dynamically from the quantum fields (matter source) propagating in the that metric.

We shall successively present the three levels of study. The first stage was reviewed in the 1996 Chalonge School [11] (see [12] for further progress). The second level is the subject of secs. VII and VIII. We study the parametric and spinodal resonances both in FRW and de Sitter backgrounds wide range of initial conditions both in FRW and de Sitter backgrounds [13,15]. [Parametric resonance appears in chaotic inflationary scenarios for unbroken symmetry whereas spinodal unstabilities show up in new inflation scenarios with broken symmetry]. Both types of unstabilities shut-off through the non-linear quantum evolution as described in secs. VII and VIII 13,15 both analytically and numerically. We follow the equation of state of the quantum matter during the evolution and analyze its properties.

The third stage of our approach is to apply non-equilibrium quantum field theory techniques to the situation of a scalar field coupled to semiclassical gravity, where the source of the gravitational field is the expectation value of the stress energy tensor in the relevant, 
dynamically changing, quantum state. In this way we can go beyond the standard analyses 35 38 which treat the background as fixed and do not consider the non-linear quantum field dynamics.

In all cases $1 .-3$, the quantum fields energy- momentum tensor is covariantly conserved both at the regularized as well as the renormalized levels [10] - [16].

We mainly consider for the stage 3. new inflation scenarios where a scalar field $\phi$ evolves under the action of a typical symmetry breaking potential. The initial conditions will be taken so that the initial value of the order parameter is near the top of the potential (the disordered state) with essentially zero time derivative. What we find is that the existence of spinodal instabilities, i.e. the fact that eventually (in an expanding universe) all modes will act as if they have a negative mass squared, drives the quantum fluctuations to grow non-perturbatively large. We have the picture of an initial wave-function or density matrix peaked near the unstable state and then spreading until it samples the stable vacua. Since these vacua are non-perturbatively far from the initial state (typically $\sim m / \sqrt{\lambda}$, where $m$ is the mass scale of the field and $\lambda$ the quartic self-coupling), the spinodal instabilities will persist until the quantum fluctuations, as encoded in the equal time two-point function $\left\langle\Phi(\vec{x}, t)^{2}\right\rangle$, grow to $\mathcal{O}\left(m^{2} / \lambda\right)$.

This growth eventually shuts off the inflationary behavior of the scale factor as well as the growth of the quantum fluctuations (this last also happens in Minkowski spacetime [10 12]).

The scenario envisaged here is that of a quenched or super-cooled phase transition where the order parameter is zero or very small. Therefore one is led to ask:

a) What is rolling down?.

b) Since the quantum fluctuations are non-perturbatively large $(\sim 1 / \lambda)$, will not they modify drastically the FRW dynamics?.

c) How can one extract (small?) metric perturbations from non-perturbatively large field fluctuations?

We address the questions a)-c) as well as other issues in sec. IX.

We choose such type of new inflationary scenario because the issue of large quantum fluctuations is particularly dramatic there. However, our methods do apply to any inflationary scenario as chaotic, extended and hybrid inflation.

\section{QUANTUM FIELD THEORY AROUND AN EXCITED STATE WITH NON-PERTURBATIVE ENERGY DENSITY}

To start we present a simple study of quantum field theory around an excited state. The relevant situation both in cosmology and high energy particle scattering is when the energy density is large $(\sim \mathcal{O}(1 / \lambda)$, non-perturbative) with respect to the ground state. For simplicity we hall make the derivation in Minkowski spacetime. The generalization to cosmological spacetimes is straightforward.

Let us consider a scalar field model with quartic selfcoupling

$$
\mathcal{L}=\frac{1}{2}\left(\partial_{\mu} \Phi\right)^{2}-\frac{m^{2}}{2} \Phi^{2}-\frac{\lambda}{4 !} \Phi^{4}
$$

Here $\Phi(x)$ is a real field. The equations of motion take the form 


$$
\partial^{2} \Phi+m^{2} \Phi+\frac{\lambda}{6} \Phi^{3}=0
$$

and the canonical momentum is

$$
\Pi(x)=\frac{\partial \mathcal{L}}{\partial \dot{\Phi}}=\dot{\Phi}(x) .
$$

For classical fields, the solutions of the equations of motion (2.2) depend on the coupling constant $\lambda$ as follows,

$$
\Phi_{c}(x)=\frac{m}{\sqrt{\lambda}} F(m x)
$$

where the index ${ }_{c}$ indicates that we are considering c-number solutions of eqs.(2.2) and $F$ is a dimensionless functions. Therefore, the classical solutions are large for small coupling and the energy density

$$
\mathcal{H}=\frac{1}{2}\left[\dot{\Phi}^{2}+(\nabla \Phi)^{2}\right]+\frac{m^{2}}{2} \Phi^{2}+\frac{\lambda}{4 !} \Phi^{4}
$$

scales as $m^{4} / \lambda$ and is large too. Notice that here $\lambda \sim \hbar$. Therefore, for small $\lambda$ we should expect some semiclassical behavior.

Let us now consider the quantum field $\Phi(x) . \Phi(x)$ and $\Pi(x)$ will be operators in a Fock space obeying the canonical commutation rules,

$$
[\Phi(\vec{x}, t), \Pi(\vec{y}, t)]=i \delta(\vec{x}-\vec{y})
$$

Let us consider a quantum state $\mid>$ which is not the vacuum. The expectation value of $\Phi(x)$ there

$$
\phi(x) \equiv<\Phi(x)>
$$

will in general be a function of space and time. $\phi(x)$ will be typically of order $\frac{m}{\sqrt{\lambda}}$ for small $\lambda$ just for correspondence with the classical theory.

We write the operator $\Phi(x)$ as,

$$
\Phi(x)=\phi(x)+\psi(x)
$$

where $\psi(x)$ is a new quantum operator. $\psi(x)$ must obey the constraint

$$
<\psi(x)>=0 \text {. }
$$

The equations of motion (2.2) hold in the quantum theory for the operator $\Phi(x)$. Taking the expectation value of eq.(2.2) in the state $\mid>$ yields

$$
\left(\partial^{2}+m^{2}\right) \phi(x)+\frac{\lambda}{6} \phi(x)^{3}+\frac{\lambda}{2} \phi(x)<\psi(x)^{2}>+\frac{\lambda}{6}<\psi(x)^{3}>=0 .
$$

Subtracting eq.(2.5) from eq.(2.2) yields,

$$
\left[\partial^{2}+m^{2}+\frac{\lambda}{2} \psi(x)^{2}\right] \psi(x)+\frac{\lambda}{2} \phi(x)\left[\psi(x)^{2}-<\psi(x)^{2}>\right]+\frac{\lambda}{6}\left[\psi(x)^{3}-<\psi(x)^{3}>\right]=0 \text {. }
$$


In order to solve (2.6) perturbatively in $\lambda$ but keeping $\phi(x)$ arbitrary, we introduce the Green function,

$$
\left[\partial^{2}+m^{2}+\frac{\lambda}{2} \phi(x)^{2}\right] G(x, y)=\delta^{4}(x-y) .
$$

We consider the retarded Green function which vanishes for $x^{0}<y^{0}$. This Green function (propagator) depends on the field expectation value $\phi(x)$ showing that the particle propagation depends here on the properties of the state. As is well known, this is not the case on the vacuum where the propagator is explicitly known.

With the help of this retarded Green function, we can write the exact evolution equation (2.6) as follows

$$
\psi(x)=\psi_{0}(x)-\frac{\lambda}{6} \int d^{4} y G(x, y)\left\{3 \phi(y)\left[\psi(y)^{2}-<\psi(y)^{2}>\right]+\psi(y)^{3}-<\psi(y)^{3}>\right\}
$$

Here, $\psi_{0}(x)$ stands for the general operator solution of the homogeneous equation:

$$
\left[\partial^{2}+m^{2}+\frac{\lambda}{2} \phi(x)^{2}\right] \psi_{0}(x)=0
$$

with $\left\langle\psi_{0}(x)>=0 . \quad \psi_{0}(x)\right.$ will be expressed as a sum of c-number eigenfunctions times creation and annihilation operators (see below eq.(2.10). It then follows that

$$
<\psi_{0}(x)^{2}>\neq 0 \text { but }<\psi_{0}(x)^{3}>=0 \text {. }
$$

For small $\lambda$ we see from eq.(2.7) that

$$
\psi(x)=\psi_{0}(x)+\mathcal{O}(\lambda)
$$

Inserting that information into eq.(2.5) yields,

$$
\left[\partial^{2}+m^{2}+\frac{\lambda}{2}<\psi_{0}(x)^{2}>\right] \phi_{0}(x)+\lambda \phi_{0}(x)^{3}=0,
$$

where,

$$
\phi(x)=\phi_{0}(x)+\mathcal{O}(\lambda) .
$$

In addition, we can consider $\psi_{0}(x)$ as the general operator solution of the equation

$$
\left[\partial^{2}+m^{2}+\frac{\lambda}{2} \phi_{0}(x)^{2}\right] \psi_{0}(x)=0
$$

Let us consider from now on spatially homogeneous states $\mid>$. Then,

$$
<\Phi(x)>=\phi(t)
$$

only depends on time. This is the relevant situation for early universe investigations. We can now Fourier expand $\psi_{0}(x)$ as follows,

$$
\psi_{0}(\vec{x}, t)=\int \frac{d^{3} k}{\sqrt{2}(2 \pi)^{3 / 2}}\left[a_{\vec{k}} f_{k}(t) e^{i \vec{k} \cdot \vec{x}}+a_{\vec{k}}^{\dagger} f_{k}^{*}(t) e^{-i \vec{k} \cdot \vec{x}}\right]
$$


Here, $a_{\vec{k}}^{\dagger}$ and $a_{\vec{k}}$ stand for creation and annihilation operators on the state $\mid>$. That is,

$$
a_{\vec{k}} \mid>=0 \quad \text { and } \quad a_{\vec{k}}^{\dagger} \mid>\neq 0 \quad \text { for } \quad \text { all } \vec{k}
$$

and

$$
\left[a_{\vec{k}}, a_{\vec{k}^{\prime}}^{\dagger}\right]=\delta\left(\vec{k}-\vec{k}^{\prime}\right)
$$

The mode functions $f_{k}(t)$ have here a non-trivial time dependence. [Recall that they have just harmonic time dependence around the vacuum]. Actually, most of the field dynamics is encoded in their evolution defined by eq.(2.9)

$$
\left[\frac{d^{2}}{d t^{2}}+k^{2}+m^{2}+\frac{\lambda}{2} \phi_{0}(t)^{2}\right] f_{k}(t)=0
$$

The canonical momentum (2.3) is Fourier expanded as follows,

$$
\pi_{0}(\vec{x}, t)=\int \frac{d^{3} k}{\sqrt{2}(2 \pi)^{3 / 2}}\left[a_{\vec{k}} \dot{f}_{k}(t) e^{i \vec{k} \cdot \vec{x}}+a_{\vec{k}}^{\dagger} \dot{f}_{k}^{*}(t) e^{-i \vec{k} \cdot \vec{x}}\right]
$$

It is easy to show now that the canonical commutation rules (2.4) hold using eq.(2.12) and the time independence of the Wronskian that we normalize as follows:

$$
W\left[f_{k}(t), f_{k}^{*}(t)\right] \equiv f_{k}(t) \dot{f}_{k}^{*}(t)-f_{k}^{*}(t) \dot{f}_{k}(t)=2 i
$$

It is then convenient to choose as initial conditions for the mode functions:

$$
f_{k}(0)=\frac{1}{\sqrt{\omega_{k}}} \quad, \quad \dot{f}_{k}(0)=-i \sqrt{\omega_{k}}
$$

with

$$
\omega_{k} \equiv \sqrt{k^{2}+m^{2}+\frac{\lambda}{2} \phi(0)^{2}}
$$

One must specify in addition the values of

$$
\phi(0)=\phi_{0} \quad \text { and } \quad \dot{\phi}(0)=p_{0}
$$

We can now compute the expectation value $<\psi_{0}(x)^{2}>$ that appears in eq.(2.8). We get using eqs.2.10), (2.11) and (2.12,

$$
<\psi_{0}(x)^{2}>=\int \frac{d^{3} k}{(2 \pi)^{3}}\left|f_{k}(t)\right|^{2}=\int \frac{k^{2} d k}{2 \pi^{2}}\left|f_{k}(t)\right|^{2} .
$$

This integral diverges in the ultraviolet. We put for the moment a cutoff $\Lambda$ in the momentum. We shall discuss below (sec. V) how the cutoff dependence can be absorbed into mass a coupling constant renormalization.

In summary, we have obtained a set of self-consistent evolution equations for the quantum state $\mid>$ : 


$$
\begin{aligned}
& {\left[\frac{d^{2}}{d t^{2}}+k^{2}+m^{2}+\frac{\lambda}{2} \phi_{0}(t)^{2}\right] f_{k}(t)=0,} \\
& {\left[\frac{d^{2}}{d t^{2}}+m^{2}+\frac{\lambda}{2} \int_{0}^{\Lambda} \frac{k^{\prime 2} d k^{\prime}}{2 \pi^{2}}\left|f_{k^{\prime}}(t)\right|^{2}\right] \phi_{0}(t)+\lambda \phi_{0}(t)^{3}=0,}
\end{aligned}
$$

with the initial conditions defined by eqs. 2.14)-(2.15). The choice of the initial conditions defines the state $\mid>$. We have an infinite number of unknowns:

$$
f_{k}(t), \phi_{0}(t) \text { for } 0 \leq k<\infty \text { and } t>0 \text {. }
$$

defined by the coupled nonlinear equations (2.16).

All physical quantities can be computed in terms of the mode functions $f_{k}(t), 0 \leq k<\infty$ and the order parameter $\phi_{0}(t)$. We have described a small coupling or self-consistent oneloop approximation. The large $N$ and Hartree approximations are discussed in sec. IV. Large $N$ and Hartree contain definitely more information than the self-consistent one-loop approximation (2.16). However, the simplicity of its derivation makes it a very useful and pedagogical exercise.

\section{NON-EQUILIBRIUM QUANTUM FIELD THEORY, SEMICLASSICAL GRAVITY AND INFLATION}

We present here the framework of the non-equilibrium closed time path formalism. For a more complete discussion, the reader is referred to [10], or the alternative approach given in 13$]$.

The time evolution of a system is determined in the Schrödinger picture by the functional Liouville equation

$$
i \frac{\partial \rho(t)}{\partial t}=[H(t), \rho(t)]
$$

where $\rho$ is the density matrix and we allow for an explicitly time dependent Hamiltonian as is

necessary to treat quantum fields in a time dependent background. Formally, the solutions to this equation for the time evolving density matrix are given by the time evolution operator, $U\left(t, t^{\prime}\right)$, in the form

$$
\rho(t)=U\left(t, t_{0}\right) \rho\left(t_{0}\right) U^{-1}\left(t, t_{0}\right) .
$$

The quantity $\rho\left(t_{0}\right)$ determines the initial condition for the evolution. We choose this initial condition to describe a state of local equilibrium in conformal time, which is also identified with the conformal adiabatic vacuum for short wavelengths. In the appendix we provide an analysis and discussion of different initial conditions and their physical content within the context of expanding cosmologies.

Given the evolution of the density matrix (3.2), ensemble averages of operators are given by the expression (again in the Schrödinger picture)

$$
\langle\mathcal{O}(t)\rangle=\frac{\operatorname{Tr}\left[U\left(t_{0}, t\right) \mathcal{O} U\left(t, t^{\prime}\right) U\left(t^{\prime}, t_{0}\right) \rho\left(t_{0}\right)\right]}{\operatorname{Tr} \rho\left(t_{0}\right)},
$$


where we have inserted the identity, $U\left(t, t^{\prime}\right) U\left(t^{\prime}, t\right)$ with $t^{\prime}$ an arbitrary time which will be taken to infinity. The state is first evolved forward from the initial time $t_{0}$ to $t$ when the operator is inserted. We then evolve this state forward to time $t^{\prime}$ and back again to the initial time.

The actual evolution of various quantities in the theory can now be evaluated by either constructing the appropriate Green functions as in [10], or by choosing an explicit Ansatz for the functional form of the time dependent density matrix so that the trace in (3.3) may be explicitly evaluated as a functional integral (see [13]). The methods are equivalent, and provide the results which will be presented below for the cases of interest.

We shall study the inflationary dynamics in a spatially flat Friedmann-Robertson-Walker background with scale factor $a(t)$ and line element:

$$
d s^{2}=d t^{2}-a^{2}(t) d \vec{x}^{2} .
$$

Our Lagrangian density has the form

$$
\mathcal{L}=\sqrt{-g}\left[\frac{1}{2} \nabla_{\mu} \Phi \nabla^{\mu} \Phi-V(\Phi)\right]
$$

Our approach can be generalized to open as well as closed cosmologies.

Our program incorporates the non-equilibrium behavior of the quantum fields involved in inflation into a framework where the geometry (gravity) is dynamical and is treated self consistently. We do this via the use of semiclassical gravity [30] where we say that the metric is classical and determined through the Einstein equations using the expectation value of the stress energy tensor $\left\langle T_{\mu \nu}\right\rangle$. Such expectation value is taken in the dynamically determined state described by the density matrix $\rho(t)$. This dynamical problem can be described schematically as follows:

1. The dynamics of the scale factor $a(t)$ is driven by the semiclassical Einstein equations

$$
\frac{1}{8 \pi G_{R}} G_{\mu \nu}+\frac{\Lambda_{R}}{8 \pi G_{R}} g_{\mu \nu}+(\text { higher curvature })=-\left\langle T_{\mu \nu}\right\rangle_{R} .
$$

Here $G_{R}, \Lambda_{R}$ are the renormalized values of Newton's constant and the cosmological constant, respectively and $G_{\mu \nu}$ is the Einstein tensor. The higher curvature terms must be included to absorb ultraviolet divergences.

2. On the other hand, the density matrix $\rho(t)$ of the matter (that determines $\left\langle T_{\mu \nu}\right\rangle_{R}$ ) obeys the Liouville equation

$$
i \frac{\partial \rho(t)}{\partial t}=[H, \rho(t)]
$$

where $H$ is the evolution Hamiltonian, which is dependent on the scale factor, $a(t)$.

It is this set of equations we must try to solve; it is clear that initial conditions must be appended to these equations for us to be able to arrive at unique solutions to them. Let us discuss some aspects of the initial state of the field theory first. 


\section{A. On the initial state: dynamics of phase transitions}

The situations we consider are

1. the theory admits a symmetry breaking potential and in which the field expectation value starts its evolution near the unstable point.

2. The symmetry is not broken and the field expectation value starts its evolution at a finite distance from the absolute minimum.

There is an issue as to how the field got to have an expectation value near the unstable point (typically at $\Phi=0$ ) as well as an issue concerning the initial state of the non-zero momentum modes.

Since our background is an FRW spacetime, it is spatially homogeneous and we can choose our state $\rho(t)$ to respect this symmetry. Starting from the full quantum field $\Phi(\vec{x}, t)$ we can extract a part that has a natural interpretation as the zero momentum, c-number part of the field by writing:

$$
\begin{aligned}
\Phi(\vec{x}, t) & =\phi(t)+\Psi(\vec{x}, t) \\
\phi(t) & =\operatorname{Tr}[\rho(t) \Phi(\vec{x}, t)] \equiv\langle\Phi(\vec{x}, t))\rangle .
\end{aligned}
$$

The quantity $\Psi(\vec{x}, t)$ represents the quantum fluctuations about the zero mode $\phi(t)$ and clearly satisfies $\langle\Psi(\vec{x}, t)\rangle=0$.

We need to choose a basis to represent the density matrix. A natural choice consistent with the translational invariance of our quantum state is that given by the Fourier modes, in comoving momentum space, of the quantum fluctuations $\Psi(\vec{x}, t)$ :

$$
\Psi(\vec{x}, t)=\int \frac{d^{3} k}{(2 \pi)^{3}} \exp (-i \vec{k} \cdot \vec{x}) \psi_{k}(t)
$$

In this language we can state our ansatz for the initial condition of the quantum state as follows. We take the zero mode $\phi(t=0)=\phi_{0}, \dot{\phi}(t=0)=0$, where $\phi_{0}$ will typically be very near the origin for broken symmetry and at a finite distance from it in the unbroken symmetry case. The initial conditions on the the nonzero modes $\psi_{k}(t=0)$ will be chosen such that the initial density matrix $\rho(t=0)$ describes a vacuum state (i.e. an initial state in local thermal equilibrium at a temperature $\left.T_{i}=0\right)$. There are some subtleties involved in this choice. First, as explained in [14], in order for the density matrix to commute with the initial Hamiltonian, we must choose the modes to be initially in the conformal adiabatic vacuum (these statements will be made more precise below). This choice has the added benefit of allowing for time independent renormalization counterterms to be used in renormalizing the theory.

We are making the assumption of an initial vacuum state in order to be able to proceed with the calculation. It would be interesting to understand what forms of the density matrix can be used for other more general initial conditions.

The assumptions of an initial equilibrium vacuum state are essentially the same used in refs. 35], 36] and [38 in the analysis of the quantum mechanics of inflation in a fixed de Sitter background. 
As discussed in the introduction, if we start from such an initial state, spinodal or parametric instabilities will drive the growth of non-perturbatively large quantum fluctuations. In order to deal with these, we need to be able to perform calculations that take these large fluctuations into account. Although the quantitative features of the dynamics will depend on the initial state, the qualitative features associated with spinodal or parametric unstabilities are fairly robust for a wide choice of initial states that describe a phase transition.

\section{THE INFLATON MODEL AND THE EQUATIONS OF MOTION}

Having recognized the appearance of large quantum fluctuations driven by parametric or spinodal unstabilities, we need to study the dynamics within a non-perturbative framework. That is, a framework allowing calculations for non-perturbatively large energy densities. We require that such a framework be: i) renormalizable, ii) covariant energy conserving, iii) numerically implementable. There are very few schemes that fulfill all of these criteria: the Hartree and the large $N$ approximation [10]- [16]. Whereas the Hartree approximation is basically a Gaussian variational approximation [29] that in general cannot be consistently improved upon, the large $N$ approximation can be consistently implemented beyond leading order [39,40]. In addition, the presence of a large number of fields in most of the GUT's models suggest that the large $N$ limit will be actually a realistic one. Moreover, for the case of broken symmetry it has the added bonus of providing many light fields (associated with Goldstone modes) that will permit the study of the effects of other fields which are lighter than the inflaton on the dynamics. Thus we will study the inflationary dynamics within the framework of the large $N$ limit of a scalar theory in the vector representation of $O(N)$ both for unbroken and broken symmetry. In the second case we will have a quenched phase transition.

We assume that the universe is spatially flat with a metric given by eq.(3.4). The matter action and Lagrangian density are given by eq.(3.5),

$$
\begin{gathered}
S_{m}=\int d^{4} x \mathcal{L}_{m}=\int d^{4} x a^{3}(t)\left[\frac{1}{2} \dot{\vec{\Phi}}^{2}(x)-\frac{1}{2} \frac{(\vec{\nabla} \vec{\Phi}(x))^{2}}{a^{2}(t)}-V(\vec{\Phi}(x))\right] \\
V(\vec{\Phi})=\frac{m^{2}}{2} \vec{\Phi}^{2}+\frac{\lambda}{8 N}\left(\vec{\Phi}^{2}\right)^{2}+\frac{1}{2} \xi \mathcal{R} \vec{\Phi}^{2}
\end{gathered}
$$

where $m^{2}>0$ for unbroken symmetry and $m^{2}<0$ for broken symmetry. Here $\mathcal{R}(t)$ stands for the scalar curvature

$$
\mathcal{R}(t)=6\left(\frac{\ddot{a}(t)}{a(t)}+\frac{\dot{a}^{2}(t)}{a^{2}(t)}\right),
$$

The coupling of $\Phi(x)$ to the scalar curvature $\mathcal{R}(t)$ has been included since arises anyhow as a consequence of renormalization [13].

The gravitational sector includes the usual Einstein term in addition to a higher order curvature term and a cosmological constant term which are necessary to renormalize the theory. The action for the gravitational sector is therefore: 


$$
S_{g}=\int d^{4} x \mathcal{L}_{g}=\int d^{4} x a^{3}(t)\left[\frac{\mathcal{R}(t)}{16 \pi G}+\frac{\alpha}{2} \mathcal{R}^{2}(t)-K\right] .
$$

with $K$ being the cosmological constant (we use $K$ rather than the conventional $\Lambda / 8 \pi G$ to distinguish the cosmological constant from the ultraviolet cutoff $\Lambda$ we introduce to regularize the theory; see section V). In principle, we also need to include the terms $R^{\mu \nu} R_{\mu \nu}$ and $R^{\alpha \beta \mu \nu} R_{\alpha \beta \mu \nu}$ as they are also terms of fourth order in derivatives of the metric (fourth adiabatic order), but the variations resulting from these terms turn out not to be independent of that of $\mathcal{R}^{2}$ in the flat FRW cosmology we are considering.

The variation of the action $S=S_{g}+S_{m}$ with respect to the metric $g_{\mu \nu}$ gives us Einstein's equation

$$
\frac{G_{\mu \nu}}{8 \pi G}+\alpha H_{\mu \nu}+K g_{\mu \nu}=-<T_{\mu \nu}>
$$

where $G_{\mu \nu}$ is the Einstein tensor given by the variation of $\sqrt{-g} \mathcal{R}, H_{\mu \nu}$ is the higher order curvature term given by the variation of $\sqrt{-g} \mathcal{R}^{2}$, and $T_{\mu \nu}$ is the contribution from the matter Lagrangian. With the metric (3.4), the various components of the curvature tensors in terms of the scale factor are:

$$
\begin{aligned}
& G_{0}^{0}=-3(\dot{a} / a)^{2}, G_{\mu}^{\mu}=-\mathcal{R}=-6\left(\frac{\ddot{a}}{a}+\frac{\dot{a}^{2}}{a^{2}}\right), \\
& H_{0}^{0}=-6\left(\frac{\dot{a}}{a} \dot{\mathcal{R}}+\frac{\dot{a}^{2}}{a^{2}} \mathcal{R}-\frac{1}{12} \mathcal{R}^{2}\right), H_{\mu}^{\mu}=-6\left(\ddot{\mathcal{R}}+3 \frac{\dot{a}}{a} \dot{\mathcal{R}}\right) .
\end{aligned}
$$

Eventually, when we have fully renormalized the theory, we will set $\alpha_{R}=0$ and keep as our only contribution to $K_{R}$ a piece related to the matter fields which we shall incorporate into $T_{\mu \nu}$.

\section{A. The Large $N$ Approximation}

To obtain the proper large $N$ limit, the inflaton field is written as

$$
\vec{\Phi}(\vec{x}, t)=(\sigma(\vec{x}, t), \vec{\pi}(\vec{x}, t))
$$

with $\vec{\pi}$ an $(N-1)$-plet, and we write

$$
\sigma(\vec{x}, t)=\sqrt{N} \phi(t)+\chi(\vec{x}, t) ;\langle\sigma(\vec{x}, t)\rangle=\sqrt{N} \phi(t) ;\langle\chi(\vec{x}, t)\rangle=0 .
$$

To implement the large $N$ limit in a consistent manner, one may introduce an auxiliary field as in [40]. However, the leading order contribution can be obtained equivalently by invoking the factorization [15,14]:

$$
\begin{aligned}
\chi^{4} & \rightarrow 6\left\langle\chi^{2}\right\rangle \chi^{2}+\text { constant }, \chi^{3} \rightarrow 3\left\langle\chi^{2}\right\rangle \chi, \\
(\vec{\pi} \cdot \vec{\pi})^{2} & \rightarrow 2\left\langle\vec{\pi}^{2}\right\rangle \vec{\pi}^{2}-\left\langle\vec{\pi}^{2}\right\rangle^{2}+\mathcal{O}(1 / N), \\
\vec{\pi}^{2} \chi^{2} & \rightarrow\left\langle\vec{\pi}^{2}\right\rangle \chi^{2}+\vec{\pi}^{2}\left\langle\chi^{2}\right\rangle, \vec{\pi}^{2} \chi \rightarrow\left\langle\vec{\pi}^{2}\right\rangle \chi .
\end{aligned}
$$


To obtain a large $N$ limit, we define [15, 14]

$$
\vec{\pi}(\vec{x}, t)=\psi(\vec{x}, t) \overbrace{(1,1, \cdots, 1)}^{N-1}
$$

where the large $N$ limit is implemented by the requirement that

$$
\left\langle\psi^{2}\right\rangle \approx \mathcal{O}(1),\left\langle\chi^{2}\right\rangle \approx \mathcal{O}(1), \phi \approx \mathcal{O}(1)
$$

The leading contribution is obtained by neglecting the $\mathcal{O}(1 / N)$ terms in the $N \rightarrow \infty$ limit. The resulting Lagrangian density is quadratic, with linear terms in $\chi$ and $\vec{\pi}$. The equations of motion are obtained by imposing the tadpole conditions $\langle\chi(\vec{x}, t)\rangle=0$ and $\langle\vec{\pi}(\vec{x}, t)\rangle=0$ which in this case are tantamount to requiring that the linear terms in $\chi$ and $\vec{\pi}$ in the Lagrangian density vanish. Since the action is quadratic, the quantum fields can be expanded in terms of creation and annihilation operators and mode functions that obey the Heisenberg equations of motion

$$
\vec{\pi}(\vec{x}, t)=\int \frac{d^{3} k}{(2 \pi)^{3}}\left[\vec{a}_{k} f_{k}(t) e^{i \vec{k} \cdot \vec{x}}+\vec{a}_{k}^{\dagger} f_{k}^{*}(t) e^{-i \vec{k} \cdot \vec{x}}\right]
$$

We see that since there are $N-1$ 'pion' fields, contributions from the field $\chi$ can be neglected in the $N \rightarrow \infty$ limit as they are of order $1 / N$ with respect those of $\psi$ and $\phi$.

The tadpole condition leads to the following equations of motion [15, 14]:

$$
\ddot{\phi}(t)+3 H(t) \dot{\phi}(t)+\mathcal{M}^{2}(t) \phi(t)=0,
$$

with the mode functions

$$
\left[\frac{d^{2}}{d t^{2}}+3 H(t) \frac{d}{d t}+\frac{k^{2}}{a^{2}(t)}+\mathcal{M}^{2}(t)\right] f_{k}(t)=0,
$$

where

$$
\mathcal{M}^{2}(t)=m^{2}+\xi \mathcal{R}(t)+\frac{\lambda}{2} \phi^{2}(t)+\frac{\lambda}{2}\left\langle\psi^{2}(t)\right\rangle
$$

An important point to note in the large $N$ equations of motion is that the form of the equation for the zero mode (4.11) is the same as for the $k=0$ mode function (4.12). It will be this identity that allows solutions of these equations in a symmetry broken scenario to satisfy Goldstone's theorem.

In this leading order in $1 / N$ the theory becomes Gaussian, but with the self-consistency condition

$$
\left\langle\psi^{2}(t)\right\rangle=\int \frac{d^{3} k}{2(2 \pi)^{3}}\left|f_{k}(t)\right|^{2} .
$$

The initial conditions on the modes $f_{k}(t)$ must now be determined. At this stage it proves illuminating to pass to conformal time variables in terms of the conformally rescaled fields (see [14 and section VI for a discussion) in which the mode functions obey an equation which is very similar to that of harmonic oscillators with time dependent frequencies in 
Minkowski space-time. It has been realized that different initial conditions on the mode functions lead to different renormalization counterterms [14]; in particular imposing initial conditions in comoving time leads to counterterms that depend on these initial conditions. Thus we chose to impose initial conditions in conformal time in terms of the conformally rescaled mode functions leading to the following initial conditions in comoving time:

$$
f_{k}\left(t_{0}\right)=\frac{1}{\sqrt{W_{k}}}, \quad \dot{f}_{k}\left(t_{0}\right)=\left[-\frac{\dot{a}\left(t_{0}\right)}{a\left(t_{0}\right)}-i W_{k}\right] f_{k}\left(t_{0}\right),
$$

with

$$
W_{k}^{2} \equiv k^{2}+\mathcal{M}^{2}\left(t_{0}\right)-\frac{\mathcal{R}\left(t_{0}\right)}{6} .
$$

For convenience, we have set $a\left(t_{0}\right)=1$ in eq.(4.16). At this point we recognize that when $\mathcal{M}^{2}\left(t_{0}\right)-\mathcal{R}\left(t_{0}\right) / 6<0$ the above initial condition must be modified to avoid imaginary frequencies, which are the signal of instabilities for long wavelength modes in the broken symmetry case. Thus we define the initial frequencies that determine the initial conditions (4.15) as

$$
\begin{aligned}
& W_{k}^{2} \equiv k^{2}+\left|\mathcal{M}^{2}\left(t_{0}\right)-\frac{\mathcal{R}\left(t_{0}\right)}{6}\right| \text { for } k^{2}<\left|\mathcal{M}^{2}\left(t_{0}\right)-\frac{\mathcal{R}\left(t_{0}\right)}{6}\right|, \\
& W_{k}^{2} \equiv k^{2}+\mathcal{M}^{2}\left(t_{0}\right)-\frac{\mathcal{R}\left(t_{0}\right)}{6} \text { for } k^{2} \geq\left|\mathcal{M}^{2}\left(t_{0}\right)-\frac{\mathcal{R}\left(t_{0}\right)}{6}\right| .
\end{aligned}
$$

In the unbroken symmetry case $\left(m^{2}>0\right)$ we use eq.(4.18) for all $k$.

As an alternative we have also used initial conditions which smoothly interpolate from positive frequencies for the unstable modes to the adiabatic vacuum initial conditions defined by (4.15)-(4.16) for the high $k$ modes. While the alternative choices of initial conditions result in small quantitative differences in the results (a few percent in quantities which depend strongly on these low- $k$ modes), all of the qualitative features we will examine are independent of this choice.

In the large $N$ limit we find the energy density and pressure density to be given by [15.14]

$$
\begin{aligned}
\frac{\varepsilon}{N} & =\frac{1}{2} \dot{\phi}^{2}+\frac{1}{2} m^{2} \phi^{2}+\frac{\lambda}{8} \phi^{4}+\frac{m^{4}}{2 \lambda}-\xi G_{0}^{0} \phi^{2}+6 \xi \frac{\dot{a}}{a} \phi \dot{\phi} \\
& +\frac{1}{2}\left\langle\dot{\psi}^{2}\right\rangle+\frac{1}{2 a^{2}}\left\langle(\nabla \psi)^{2}\right\rangle+\frac{1}{2} m^{2}\left\langle\psi^{2}\right\rangle+\frac{\lambda}{8}\left[2 \phi^{2}\left\langle\psi^{2}\right\rangle+\left\langle\psi^{2}\right\rangle^{2}\right] \\
& -\xi G_{0}^{0}\left\langle\psi^{2}\right\rangle+6 \xi \frac{\dot{a}}{a}\langle\psi \dot{\psi}\rangle, \\
\frac{\varepsilon-3 p}{N} & =-\dot{\phi}^{2}+2 m^{2} \phi^{2}+\frac{\lambda}{2} \phi^{4}+\frac{2 m^{4}}{\lambda}-\xi G_{\mu}^{\mu} \phi^{2}+6 \xi\left(\phi \ddot{\phi}+\dot{\phi}^{2}+3 \frac{\dot{a}}{a} \phi \dot{\phi}\right) \\
& -\left\langle\dot{\psi}^{2}\right\rangle+\frac{1}{a^{2}}\left\langle(\nabla \psi)^{2}\right\rangle+2 m^{2}\left\langle\psi^{2}\right\rangle-\xi G_{\mu}^{\mu}\left\langle\psi^{2}\right\rangle \\
& +\frac{\lambda}{2}\left[2 \phi^{2}\left\langle\psi^{2}\right\rangle+\left\langle\psi^{2}\right\rangle^{2}\right]+6 \xi\left(\langle\psi \ddot{\psi}\rangle+\left\langle\dot{\psi}^{2}\right\rangle+3 \frac{\dot{a}}{a}\langle\psi \dot{\psi}\rangle\right),
\end{aligned}
$$

where $\left\langle\psi^{2}\right\rangle$ is given by equation (4.14) and we have defined the following integrals: 


$$
\begin{aligned}
\left\langle(\nabla \psi)^{2}\right\rangle & =\int \frac{d^{3} k}{2(2 \pi)^{3}} k^{2}\left|f_{k}(t)\right|^{2}, \\
\left\langle\dot{\psi}^{2}\right\rangle & =\int \frac{d^{3} k}{2(2 \pi)^{3}}\left|\dot{f}_{k}(t)\right|^{2} .
\end{aligned}
$$

The composite operators $\langle\psi \dot{\psi}\rangle$ and $\langle\psi \ddot{\psi}\rangle$ are symmetrized by removing a normal ordering constant to yield

$$
\begin{aligned}
& \frac{1}{2}(\langle\psi \dot{\psi}\rangle+\langle\dot{\psi} \psi\rangle)=\frac{1}{4} \int \frac{d^{3} k}{(2 \pi)^{3}} \frac{d\left|f_{k}(t)\right|^{2}}{d t} \\
& \frac{1}{2}(\langle\psi \ddot{\psi}\rangle+\langle\ddot{\psi} \psi\rangle)=\frac{1}{4} \int \frac{d^{3} k}{(2 \pi)^{3}}\left[f_{k}(t) \ddot{f}_{k}^{*}(t)+\ddot{f}_{k}(t) f_{k}^{*}(t)\right] .
\end{aligned}
$$

The last of these integrals, (4.24), may be rewritten using the equation of motion (4.12):

$$
\langle\psi \ddot{\psi}\rangle=-3 \frac{\dot{a}}{a}\langle\psi \dot{\psi}\rangle-\frac{\left\langle(\nabla \psi)^{2}\right\rangle}{a^{2}}-\mathcal{M}^{2}\left\langle\psi^{2}\right\rangle
$$

It is straightforward to show that the bare energy is covariantly conserved by using the equations of motion for the zero mode and the mode functions.

\section{B. Hartree Approximation}

In the Hartree approximation, our theory is that of a single component scalar field, $\Phi(\vec{x}, t)$, with the $Z_{2}$ symmetry $\Phi \rightarrow-\Phi$. The potential can be written as:

$$
V(\Phi)=\frac{1}{2}\left(m^{2}+\xi \mathcal{R}\right) \Phi^{2}+\frac{\lambda}{4 !} \Phi^{4}
$$

where $\mathcal{R}$ is the Ricci scalar. We have rescaled the coupling constant here by a factor 3 compared with the one used in the large $N$ limit [eq.(4.2)].

We decompose the field into its zero mode, $\phi(t)=\langle\Phi(\vec{x}, t)\rangle$, fluctuations $\psi(\vec{x}, t)$ about it:

$$
\Phi(\vec{x}, t)=\phi(t)+\psi(\vec{x}, t) .
$$

The potential (4.26) may then be expanded in terms of these fields.

The Hartree approximation is achieved by making the potential quadratic in the fluctuation field $\psi$ by invoking the factorization

$$
\begin{aligned}
& \psi^{3}(\vec{x}, t) \rightarrow 3\left\langle\psi^{2}(\vec{x}, t)\right\rangle \psi(\vec{x}, t), \\
& \psi^{4}(\vec{x}, t) \rightarrow 6\left\langle\psi^{2}(\vec{x}, t)\right\rangle \psi^{2}(\vec{x}, t)-3\left\langle\psi^{2}(\vec{x}, t)\right\rangle^{2} .
\end{aligned}
$$

This factorization yields a quadratic theory in which the effects of interactions are encoded in the time dependent mass which is determined self-consistently.

The equations of motion for the zero mode and the fluctuations are given by the tadpole equation

$$
\langle\psi(\vec{x}, t)\rangle=0
$$


Introducing the Fourier mode functions, $U_{k}(t)$, they can be written as:

$$
\begin{gathered}
\ddot{\phi}(t)+3 \frac{\dot{a}(t)}{a(t)} \dot{\phi}(t)+\left(m^{2}+\xi \mathcal{R}(t)\right) \phi(t)+\frac{\lambda}{6} \phi^{3}(t)+\frac{\lambda}{2} \phi(t)\left\langle\psi^{2}(t)\right\rangle=0 \\
{\left[\frac{d^{2}}{d t^{2}}+3 \frac{\dot{a}(t)}{a(t)} \frac{d}{d t}+\frac{k^{2}}{a^{2}(t)}+m^{2}+\xi \mathcal{R}(t)+\frac{\lambda}{2} \phi^{2}(t)+\frac{\lambda}{2}\left\langle\psi^{2}(t)\right\rangle\right] U_{k}(t)=0} \\
\left\langle\psi^{2}(t)\right\rangle=\int \frac{d^{3} k}{2(2 \pi)^{3}}\left|U_{k}(t)\right|^{2}
\end{gathered}
$$

The initial conditions on the mode functions are the same as eq.(4.15) in the large $N$ limit,

$$
U_{k}\left(t_{0}\right)=\frac{1}{\sqrt{W_{k}}}, \quad \dot{U}_{k}\left(t_{0}\right)=\left[-\frac{\dot{a}\left(t_{0}\right)}{a\left(t_{0}\right)}-i W_{k}\right] U_{k}\left(t_{0}\right),
$$

with the frequencies $W_{k}$ given by eq. (4.16)

A detailed analysis and discussion of the choice of initial conditions and the frequencies (4.16) is provided in the sec. VI. As discussed there, this choice corresponds to the large- $k$ modes being in the conformal adiabatic vacuum state. In what follows we will subtract the composite operator $\psi^{2}(t)$ at the initial time and absorb the term $\frac{\lambda}{2}\left\langle\psi^{2}\left(t_{0}\right)\right\rangle$ in a renormalization of the mass.

Notice that we have used identical notations in the large $N$ and Hartree cases to avoid cluttering and also to stress the similarity between the two approximations. In particular, we note that the only difference in the expressions for the two cases [eqs.(4.28) and (4.11), respectively] is a factor of three appearing in the self interaction term in the equations for the zero mode.

It is instructive to compare the Hartree approximation with the self-consistently one-loop approximation presented in sec. II. We see comparing eq.(2.16) with eq.(4.27)-(4.28) that they are rather similar. The only difference being that the quantum fluctuations are present in both Hartree equations whereas they are only present in the zero mode equation in the self-consistently one-loop approximation. In fact, in the weak coupling limit, the Hartree approximation becomes the self-consistently one-loop approximation.

\section{RENORMALIZATION}

Renormalization is a very subtle but important issue in gravitational backgrounds [30. The fluctuation contribution $\left\langle\psi^{2}(\vec{x}, t)\right\rangle$, the energy, and the pressure all need to be renormalized. The renormalization aspects in curved space times have been discussed at length in the literature [30] and have been extended to the large $N$ self-consistent approximations for the non-equilibrium backreaction problem in [40,14 [16,41. More recently, a consistent and covariant regularization scheme that can be implemented numerically has been proposed [31].

In terms of the effective mass term for the large $N$ limit given by (4.13) and defining the quantity 


$$
\begin{aligned}
B(t) & \equiv a^{2}(t)\left(\mathcal{M}^{2}(t)-\mathcal{R} / 6\right), \\
\mathcal{M}^{2}(t) & =m_{B}^{2}+\xi_{B} \mathcal{R}(t)+\frac{\lambda_{B}}{2} \phi^{2}(t)+\frac{\lambda_{B}}{2}\left\langle\psi^{2}(t)\right\rangle_{B},
\end{aligned}
$$

where the subscript $B$ stands for bare quantities, we find the following large $k$ behavior for the case of an arbitrary scale factor $a(t)$ (with $a(0)=1$ ):

$$
\begin{aligned}
&\left|f_{k}(t)\right|^{2}=\frac{1}{k a^{2}(t)}-\frac{1}{2 k^{3} a^{2}(t)} B(t) \\
&+\frac{1}{8 k^{5} a^{2}(t)}\left\{3 B(t)^{2}+a(t) \frac{d}{d t}[a(t) \dot{B}(t)]\right\}+\mathcal{O}\left(1 / k^{7}\right) \\
&=\mathcal{S}^{(2)}+\mathcal{O}\left(1 / k^{5}\right), \\
&\left|\dot{f}_{k}(t)\right|^{2}=\frac{k}{a^{4}(t)}+\frac{1}{2 k a^{4}(t)}\left[B(t)+2 \dot{a}^{2}\right] \\
&+\frac{1}{8 k^{3} a^{4}(t)}\left\{-B(t)^{2}-a(t)^{2} \ddot{B}(t)+3 a(t) \dot{a}(t) \dot{B}(t)-4 \dot{a}^{2}(t) B(t)\right\}+\mathcal{O}\left(1 / k^{5}\right) \\
&=\mathcal{S}^{(1)}+\mathcal{O}\left(1 / k^{5}\right), \\
& \frac{1}{2}\left[f_{k}(t) \dot{f}_{k}^{*}(t)+\dot{f}_{k}(t) f_{k}^{*}(t)\right]=-\frac{1}{k a^{2}(t)} \frac{\dot{a}(t)}{a(t)}-\frac{1}{4 k^{3} a^{2}(t)}\left[\dot{B}(t)-2 \frac{\dot{a}(t)}{a(t)} B(t)\right]+\mathcal{O}\left(1 / k^{5}\right) .
\end{aligned}
$$

Although the divergences can be dealt with by dimensional regularization, this procedure is not well suited to numerical analysis (see however ref. [31]). We will make our subtractions using an ultraviolet cutoff, $\Lambda a(t)$, constant in physical coordinates. This guarantees that the counterterms will be time independent. The renormalization then proceeds much in the same manner as in reference [13]; the quadratic divergences renormalize the mass and the logarithmic terms renormalize the quartic coupling and the coupling to the Ricci scalar. In addition, there is a quartic divergence which renormalizes the cosmological constant while the leading renormalizations of Newton's constant and the higher order curvature coupling are quadratic and logarithmic respectively. The renormalization conditions on the mass, coupling to the Ricci scalar and coupling constant are obtained from the requirement that the frequencies that appear in the mode equations are finite [13], i.e:

$$
m_{B}^{2}+\xi_{B} \mathcal{R}(t)+\frac{\lambda_{B}}{2} \phi^{2}(t)+\frac{\lambda_{B}}{2}\left\langle\psi^{2}(t)\right\rangle_{B}=m_{R}^{2}+\xi_{R} \mathcal{R}(t)+\frac{\lambda_{R}}{2} \phi^{2}(t)+\frac{\lambda_{R}}{2}\left\langle\psi^{2}(t)\right\rangle_{R},
$$

while the renormalizations of Newton's constant, the higher order curvature coupling, and the cosmological constant are given by the condition of finiteness of the semi-classical Einstein-Friedmann equation:

$$
\frac{G_{0}^{0}}{8 \pi G_{B}}+\alpha_{B} H_{0}^{0}+K_{B} g_{0}^{0}+\left\langle T_{0}^{0}\right\rangle_{B}=\frac{G_{0}^{0}}{8 \pi G_{R}}+\alpha_{R} H_{0}^{0}+K_{R} g_{0}^{0}+\left\langle T_{0}^{0}\right\rangle_{R} .
$$

Finally we arrive at the following set of renormalizations [14]: 


$$
\begin{aligned}
\frac{1}{8 \pi N G_{R}} & =\frac{1}{8 \pi N G_{B}}-2\left(\xi_{R}-\frac{1}{6}\right) \frac{\Lambda^{2}}{16 \pi^{2}}+2\left(\xi_{R}-\frac{1}{6}\right) m_{R}^{2} \frac{\ln (\Lambda / \kappa)}{16 \pi^{2}}, \\
\frac{\alpha_{R}}{N} & =\frac{\alpha_{B}}{N}-\left(\xi_{R}-\frac{1}{6}\right)^{2} \frac{\ln (\Lambda / \kappa)}{16 \pi^{2}}, \\
\frac{K_{R}}{N} & =\frac{K_{B}}{N}-\frac{\Lambda^{4}}{16 \pi^{2}}-m_{R}^{2} \frac{\Lambda^{2}}{16 \pi^{2}}+\frac{m_{R}^{4}}{2} \frac{\ln (\Lambda / \kappa)}{16 \pi^{2}}, \\
m_{R}^{2} & =m_{B}^{2}+\lambda_{R} \frac{\Lambda^{2}}{16 \pi^{2}}-\lambda_{R} m_{R}^{2} \frac{\ln (\Lambda / \kappa)}{16 \pi^{2}}, \\
\xi_{R} & =\xi_{B}-\lambda_{R}\left(\xi_{R}-\frac{1}{6}\right) \frac{\ln (\Lambda / \kappa)}{16 \pi^{2}}, \\
\lambda_{R} & =\lambda_{B}-\lambda_{R} \frac{\ln (\Lambda / \kappa)}{16 \pi^{2}}, \\
\left\langle\psi^{2}(t)\right\rangle_{R} & =\int \frac{d^{3} k}{2(2 \pi)^{3}}\left\{\left|f_{k}(t)\right|^{2}-\frac{1}{k a^{2}(t)}+\frac{\Theta(k-\kappa)}{2 k^{3}}\left[\mathcal{M}^{2}(t)-\frac{\mathcal{R}(t)}{6}\right]\right\} .
\end{aligned}
$$

Here, $\kappa$ is the renormalization point. As expected, the logarithmic terms are consistent with the renormalizations found using dimensional regularization [31,41. Again, we set $\alpha_{R}=0$ and choose the renormalized cosmological constant such that the vacuum energy is zero in the true vacuum. We emphasize that while the regulator we have chosen does not respect the covariance of the theory, the renormalized energy momentum tensor defined in this way nevertheless retains the property of covariant conservation in the limit when the cutoff is taken to infinity.

The logarithmic subtractions can be neglected because of the coupling $\lambda \leq 10^{-12}$. Using the Planck scale as the cutoff and the inflaton mass $m_{R}$ as a renormalization point, these terms are of order $\lambda \ln \left[M_{p l} / m_{R}\right] \leq 10^{-10}$, for $m \geq 10^{9} \mathrm{GeV}$. An equivalent statement is that for these values of the coupling and inflaton masses, the Landau pole is well beyond the physical cutoff $M_{p l}$. Our relative error in the numerical analysis is of order $10^{-8}$, therefore our numerical study is insensitive to the logarithmic corrections. Though these corrections are fundamentally important, numerically they can be neglected. Therefore, in the numerical computations that follow, we will neglect logarithmic renormalization and subtract only quartic and quadratic divergences in the energy and pressure, and quadratic divergences in the fluctuation contribution.

\section{A. Renormalized Equations of Motion for Dynamical Evolution in the Large N limit}

It is convenient to introduce the following dimensionless quantities and definitions,

$$
\begin{gathered}
\tau=m_{R} t \quad ; \quad h=\frac{H}{m_{R}} \quad ; \quad q=\frac{k}{m_{R}} \quad \omega_{q}=\frac{W_{k}}{m_{R}} \quad ; \quad g=\frac{\lambda_{R}}{8 \pi^{2}}, \\
\eta^{2}(\tau)=\frac{\lambda_{R}}{2 m_{R}^{2}} \phi^{2}(t) \quad ; \quad g \Sigma(\tau)=\frac{\lambda}{2 m_{R}^{2}}\left\langle\psi^{2}(t)\right\rangle_{R} \quad ; \quad f_{q}(\tau) \equiv \sqrt{m_{R}} f_{k}(t) .
\end{gathered}
$$

Choosing $\xi_{R}=0$ (minimal coupling) and the renormalization point $\kappa=\left|m_{R}\right|$ and setting $a(0)=1$, the equations of motion become for unbroken symmetry: 


$$
\begin{aligned}
& {\left[\frac{d^{2}}{d \tau^{2}}+3 h \frac{d}{d \tau}+1+\eta^{2}(\tau)+g \Sigma(\tau)\right] \eta(\tau)=0,} \\
& {\left[\frac{d^{2}}{d \tau^{2}}+3 h \frac{d}{d \tau}+\frac{q^{2}}{a^{2}(\tau)}+1+\eta^{2}+g \Sigma(\tau)\right] f_{q}(\tau)=0} \\
& f_{q}(0)=\frac{1}{\sqrt{\omega_{q}}} ; \quad \dot{f}_{q}(0)=\left[-h(0)-i \omega_{q}\right] f_{q}(0), \\
& \omega_{q}=\left[q^{2}+1+\eta^{2}(0)-\frac{\mathcal{R}(0)}{6 m_{R}^{2}}+g \Sigma(0)\right]^{\frac{1}{2}} .
\end{aligned}
$$

We find for broken symmetry,

$$
\begin{aligned}
& {\left[\frac{d^{2}}{d \tau^{2}}+3 h \frac{d}{d \tau}-1+\eta^{2}(\tau)+g \Sigma(\tau)\right] \eta(\tau)=0,} \\
& {\left[\frac{d^{2}}{d \tau^{2}}+3 h \frac{d}{d \tau}+\frac{q^{2}}{a^{2}(\tau)}-1+\eta^{2}+g \Sigma(\tau)\right] f_{q}(\tau)=0,} \\
& f_{q}(0)=\frac{1}{\sqrt{\omega_{q}}} ; \quad \dot{f}_{q}(0)=\left[-h(0)-i \omega_{q}\right] f_{q}(0), \\
& \omega_{q}=\left[q^{2}-1+\eta^{2}(0)-\frac{\mathcal{R}(0)}{6 m_{R}^{2}}+g \Sigma(0)\right]^{\frac{1}{2}} \text { for } q^{2}>1-\eta^{2}(0)+\frac{\mathcal{R}(0)}{6 m_{R}^{2}}-g \Sigma(0), \\
& \omega_{q}=\left[q^{2}+1-\eta^{2}(0)+\frac{\mathcal{R}(0)}{6 m_{R}^{2}}-g \Sigma(0)\right]^{\frac{1}{2}} \text { for } q^{2}<1-\eta^{2}(0)+\frac{\mathcal{R}(0)}{6 m_{R}^{2}}-g \Sigma(0) .
\end{aligned}
$$

Here,

$$
\Sigma(\tau)=\int_{0}^{\infty} q^{2} d q\left[\left|f_{q}(\tau)\right|^{2}-\frac{1}{q a(\tau)^{2}}+\frac{\Theta(q-1)}{2 q^{3}}\left(\frac{\mathcal{M}^{2}(\tau)}{m_{R}^{2}}-\frac{\mathcal{R}(\tau)}{6 m_{R}^{2}}\right)\right]
$$

both for unbroken and broken symmetry.

The initial conditions for $\eta(\tau)$ will be specified later. An important point to notice is that the equation of motion for the $q=0$ mode coincides with that of the zero mode (5.18). Furthermore, for $\eta(\tau \rightarrow \infty) \neq 0$, a stationary (equilibrium) solution of the eq.(5.18) is obtained for broken symmetry when the sum rule [10,11, 15, 14

$$
-1+\eta^{2}(\infty)+g \Sigma(\infty)=0
$$

is fulfilled. This sum rule is nothing but a consequence of Goldstone's theorem and is a result of the fact that the large $N$ approximation satisfies the Ward identities associated with the $O(N)$ symmetry, since the term $-1+\eta^{2}+g \Sigma$ is seen to be the effective mass of the modes transverse to the symmetry breaking direction, i.e. the Goldstone modes in the broken symmetry phase.

The renormalized dimensionless evolution equations in the Hartree approximation are very similar to eqs.(5.17)-(5.19). They can be obtained just dividing by three the $\eta^{2}$ term in the zero mode equation. [Compare with eqs.4.12)-(4.13) and (4.27)-(4.28)]. 
In terms of the zero mode $\eta(\tau)$ and the quantum mode function given by eq.(5.18) we find that the Friedmann equation for the dynamics of the scale factor in dimensionless variables is given by

$$
h^{2}(\tau)=4 h_{0}^{2} \epsilon_{R}(\tau) \quad ; \quad h_{0}^{2}=\frac{4 \pi N m_{R}^{2}}{3 M_{P l}^{2} \lambda_{R}}
$$

and the renormalized energy and pressure are given by:

$$
\begin{aligned}
\epsilon_{R}(\tau) & =\frac{1}{2} \dot{\eta}^{2}+\frac{1}{4}\left(-1+\eta^{2}+g \Sigma\right)^{2} \\
& +\frac{g}{2} \int q^{2} d q\left[\left|\dot{f}_{q}\right|^{2}-\mathcal{S}^{(1)}(q, \tau)+\frac{q^{2}}{a^{2}}\left(\left|f_{q}\right|^{2}-\Theta(q-1) \mathcal{S}^{(2)}(q, \tau)\right)\right], \\
(p+\varepsilon)_{R}(\tau) & =\frac{2 N m_{R}^{4}}{\lambda_{R}}\left\{\dot{\eta}^{2}\right. \\
& \left.+g \int q^{2} d q\left[\left|\dot{f}_{q}\right|^{2}-\mathcal{S}^{(1)}(q, \tau)+\frac{q^{2}}{3 a^{2}}\left(\left|f_{q}\right|^{2}-\Theta(q-1) \mathcal{S}^{(2)}(q, \tau)\right)\right]\right\},
\end{aligned}
$$

where the subtractions $\mathcal{S}^{(1)}$ and $\mathcal{S}^{(2)}$ are given by the right hand sides of eqs. (5.4) and (5.3) respectively.

The renormalized energy and pressure are covariantly conserved:

$$
\dot{\epsilon}_{R}(\tau)+3 h(\tau)(p+\varepsilon)_{R}(\tau)=0 .
$$

From the evolution of the mode functions that determine the quantum fluctuations, we can study the growth of correlated domains with the equal time correlation function,

$$
S(\vec{x}, t)=\langle\psi(\vec{x}, t) \psi(\overrightarrow{0}, t)\rangle=\int \frac{d^{3} k}{2(2 \pi)^{3}} e^{i \vec{k} \cdot \vec{x}}\left|f_{k}(t)\right|^{2},
$$

which can be written in terms of the power spectrum of quantum fluctuations, $\left|f_{q}(\tau)\right|^{2}$. It is convenient to define the dimensionless correlation function,

$$
\mathcal{S}(\rho, \tau)=\frac{S(|\vec{x}|, t)}{m_{R}^{2}}=\frac{1}{4 \pi^{2} \rho} \int_{0}^{\infty} q d q \sin [q \rho]\left|f_{q}(\tau)\right|^{2} ; \rho=m_{R}|\vec{x}| .
$$

We now have all the ingredients to study the particular cases of interest.

\section{CONFORMAL TIME ANALYSIS AND INITIAL CONDITIONS}

The issue of renormalization and initial conditions is best understood in conformal time which is a natural framework for adiabatic renormalization and regularization.

Quantization in conformal time proceeds by writing the metric element as

$$
d s^{2}=C^{2}(\mathcal{T})\left(d \mathcal{T}^{2}-d \vec{x}^{2}\right)
$$

Under a conformal rescaling of the field 


$$
\Phi(\vec{x}, t)=\chi(\vec{x}, \mathcal{T}) / C(\mathcal{T})
$$

the action for a scalar field (with the obvious generalization to $N$ components) becomes, after an integration by parts and dropping a surface term

$$
S=\int d^{3} x d \mathcal{T}\left\{\frac{1}{2}\left(\chi^{\prime}\right)^{2}-\frac{1}{2}(\vec{\nabla} \chi)^{2}-\mathcal{V}(\chi)\right\}
$$

with

$$
\mathcal{V}(\chi)=C^{4}(\mathcal{T}) V(\Phi / C(\mathcal{T}))-C^{2}(\mathcal{T}) \frac{\mathcal{R}}{12} \chi^{2}
$$

where $\mathcal{R}=6 C^{\prime \prime}(\mathcal{T}) / C^{3}(\mathcal{T})$ is the Ricci scalar, and primes stand for derivatives with respect to conformal time $\mathcal{T}$.

The conformal time Hamiltonian operator, which is the generator of translations in $\mathcal{T}$, is given by

$$
H_{\mathcal{T}}=\int d^{3} x\left\{\frac{1}{2} \Pi_{\chi}^{2}+\frac{1}{2}(\vec{\nabla} \chi)^{2}+\mathcal{V}(\chi)\right\}
$$

with $\Pi_{\chi}$ being the canonical momentum conjugate to $\chi, \Pi_{\chi}=\chi^{\prime}$. Separating the zero mode of the field $\chi$

$$
\chi(\vec{x}, \mathcal{T})=\chi_{0}(\mathcal{T})+\bar{\chi}(\vec{x}, \mathcal{T}),
$$

and performing the large $N$ or Hartree factorization on the fluctuations we find that the Hamiltonian becomes linear plus quadratic in the fluctuations, and similar to a Minkowski space-time Hamiltonian with a $\mathcal{T}$ dependent mass term given by

$$
\mathcal{M}^{2}(\mathcal{T})=C^{2}(\mathcal{T})\left[m^{2}+\left(\xi-\frac{1}{6}\right) \mathcal{R}+\frac{\lambda}{2} \chi_{0}^{2}(\mathcal{T})+\frac{\lambda}{2}\left\langle\bar{\chi}^{2}\right\rangle\right]
$$

We can now follow the steps and use the results of reference 13] for the conformal time evolution of the density matrix by setting $a(t)=1$ in the proper equations of that reference and replacing the frequencies by

$$
\omega_{k}^{2}(\mathcal{T})=\vec{k}^{2}+\mathcal{M}^{2}(\mathcal{T})
$$

and the expectation value in (6.7) is obtained in this $\mathcal{T}$ evolved density matrix. The time evolution of the kernels in the density matrix (see [13]) is determined by the mode functions that obey

$$
\left[\frac{d^{2}}{d \mathcal{T}^{2}}+k^{2}+\mathcal{M}^{2}(\mathcal{T})\right] F_{k}(\mathcal{T})=0
$$

The Wronskian of these mode functions

$$
\mathcal{W}\left(F, F^{*}\right)=F_{k}^{\prime} F_{k}^{*}-F_{k} F_{k}^{*^{\prime}}
$$

is a constant. It is natural to impose initial conditions such that at the initial $\mathcal{T}$ the density matrix describes a situation of local thermodynamic equilibrium and therefore commutes 
with the conformal time Hamiltonian at the initial time. This implies that the initial conditions of the mode functions $F_{k}(\mathcal{T})$ be chosen to be (see [13])

$$
F_{k}\left(\mathcal{T}_{o}\right)=\frac{1}{\sqrt{\omega_{k}\left(\mathcal{T}_{o}\right)}} ; F_{k}^{\prime}\left(\mathcal{T}_{o}\right)=-i \sqrt{\omega_{k}\left(\mathcal{T}_{o}\right)} F_{k}\left(\mathcal{T}_{o}\right)
$$

With such initial conditions, the Wronskian (6.10) takes the value

$$
\mathcal{W}\left(F, F^{*}\right)=-2 i
$$

These initial conditions correspond to the choice of mode functions which coincide with the first order adiabatic modes and those of the Bunch-Davies vacuum for large momentum [30]. To see this clearly, we write the solution of eq.(6.9) in the form,

$$
D_{k}(\mathcal{T})=e^{\int_{\mathcal{T}_{o}}^{\mathcal{T}} R_{k}\left(\mathcal{T}^{\prime}\right) d \mathcal{T}^{\prime}}
$$

with the function $R_{k}(\mathcal{T})$ obeying the Riccati equation

$$
R_{k}^{\prime}+R_{k}^{2}+k^{2}+\mathcal{M}^{2}(\mathcal{T})=0 .
$$

This equation posses the solution

$$
R_{k}(\mathcal{T})=-i k+R_{0, k}(\mathcal{T})-i \frac{R_{1, k}(\mathcal{T})}{k}+\frac{R_{2, k}(\mathcal{T})}{k^{2}}-i \frac{R_{3, k}(\mathcal{T})}{k^{3}}+\frac{R_{4, k}(\mathcal{T})}{k^{4}}+\mathcal{O} \frac{1}{k^{5}}
$$

and its complex conjugate. We find for the coefficients:

$$
\begin{aligned}
& R_{0, k}=0 ; \quad R_{1, k}=\frac{1}{2} \mathcal{M}^{2}(\mathcal{T}) ; R_{2, k}=-\frac{1}{2} R_{1, k}^{\prime} \\
& R_{3, k}=\frac{1}{2}\left(R_{2, k}^{\prime}-R_{1, k}^{2}\right) \quad ; \quad R_{4, k}=-\frac{1}{2}\left(R_{3, k}^{\prime}+2 R_{1, k} R_{2, k}\right) .
\end{aligned}
$$

The solutions $F_{k}(\mathcal{T})$ obeying the boundary conditions (6.11) are obtained as linear combinations of this WKB solution and its complex conjugate

$$
F_{k}(\mathcal{T})=\frac{1}{2 \sqrt{\omega_{k}\left(\mathcal{T}_{o}\right)}}\left[(1+\gamma) D_{k}(\mathcal{T})+(1-\gamma) D_{k}^{*}(\mathcal{T})\right]
$$

where the coefficient $\gamma$ is obtained from the initial conditions. It is straightforward to find that the real and imaginary parts are given by

$$
\gamma_{R}=1+\mathcal{O}\left(1 / k^{4}\right) ; \gamma_{I}=\mathcal{O}\left(1 / k^{3}\right)
$$

Therefore the large- $k$ mode functions satisfy the adiabatic vacuum initial conditions [30]. This, in fact, is the rationale for the choice of the initial conditions (6.11).

Following the analysis presented in [13] we find, in conformal time that

$$
\left\langle\bar{\chi}^{2}(\vec{x}, \mathcal{T})\right\rangle=\int \frac{d^{3} k}{2(2 \pi)^{3}}\left|F_{k}(\mathcal{T})\right|^{2}
$$


The Heisenberg field operators $\bar{\chi}(\vec{x}, \mathcal{T})$ and their canonical momenta $\Pi_{\chi}(\vec{x}, \mathcal{T})$ can be expanded as:

$$
\begin{aligned}
& \bar{\chi}(\vec{x}, \mathcal{T})=\int \frac{d^{3} k}{\sqrt{2}(2 \pi)^{3 / 2}}\left[a_{k} F_{k}(\mathcal{T})+a_{-k}^{\dagger} F_{k}^{*}(\mathcal{T})\right] e^{i \vec{k} \cdot \vec{x}} \\
& \Pi_{\chi}(\vec{x}, \mathcal{T})=\int \frac{d^{3} k}{\sqrt{2}(2 \pi)^{3 / 2}}\left[a_{k} F_{k}^{\prime}(\mathcal{T})+a_{-k}^{\dagger} F_{k}^{*^{\prime}}(\mathcal{T})\right] e^{i \vec{k} \cdot \vec{x}}
\end{aligned}
$$

with the time independent creation and annihilation operators $a_{k}$ and $a_{k}^{\dagger}$ obeying canonical commutation relations. Since the fluctuation fields in comoving and conformal time are related by the conformal rescaling (6.2), it is straightforward to see that the mode functions in comoving time are related to those in conformal time simply as

$$
f_{k}(t)=\frac{F_{k}(\mathcal{T})}{C(\mathcal{T})}
$$

Therefore the initial conditions (6.11) on the conformal time mode functions imply the initial conditions for the mode functions in comoving time are given by eq.(4.15).

For renormalization purposes we need the large- $k$ behavior of $\left|f_{k}(t)\right|^{2},\left|\dot{f}_{k}(t)\right|^{2}$, which are determined by the large- $k$ behavior of the conformal time mode functions and its derivative. These are given by

$$
\begin{aligned}
& \left|F_{k}(\mathcal{T})\right|^{2}=\frac{1}{k}\left[1-\frac{R_{1, k}(\mathcal{T})}{k^{2}}+\frac{1}{k^{4}}\left(\frac{R_{1, k}^{\prime \prime}(\mathcal{T})}{4}+\frac{3}{2} R_{1, k}^{2}(\mathcal{T})\right)+\mathcal{O}\left(\frac{1}{k^{6}}\right)\right] \\
& \left|F_{k}^{\prime}(\mathcal{T})\right|^{2}=k\left[1+\frac{R_{1, k}(\mathcal{T})}{k^{2}}+\frac{1}{k^{4}}\left(-\frac{R_{1, k}^{\prime \prime}(\mathcal{T})}{4}+\frac{3}{2} R_{1, k}^{2}(\mathcal{T})\right)+\mathcal{O}\left(\frac{1}{k^{6}}\right)\right] .
\end{aligned}
$$

We note that the large $k$ behavior of the mode functions to the order needed to renormalize the quadratic and logarithmic divergences is insensitive to the initial conditions. This is not the case when the initial conditions are imposed as described in [13,15]. Thus the merit in considering the initial conditions in conformal time [14].

The correspondence with the comoving time mode functions is given by:

$$
\begin{aligned}
\left|f_{k}(t)\right|^{2} & =\frac{\left|F_{k}(\mathcal{T})\right|^{2}}{C^{2}(\mathcal{T})} \\
\left|\dot{f}_{k}(t)\right|^{2} & =\frac{1}{C^{2}(\mathcal{T})}\left[\frac{\left|F_{k}^{\prime}(\mathcal{T})\right|^{2}}{C^{2}(\mathcal{T})}+\left(H^{2}-\frac{H}{C(\mathcal{T})} \frac{d}{d \mathcal{T}}\right)\left|F_{k}(\mathcal{T})\right|^{2}\right]
\end{aligned}
$$

There is an important physical consequence of this choice of initial conditions, which is revealed by analyzing the evolution of the density matrix.

In the large $N$ or Hartree (also to one-loop) approximation, the density matrix is Gaussian, and defined by a normalization factor, a complex covariance that determines the diagonal matrix elements and a real covariance that determines the mixing in the Schrödinger representation as discussed in reference [13] (and references therein).

In conformal time quantization and in the Schrödinger representation in which the field $\chi$ is diagonal the conformal time evolution of the density matrix is via the conformal time 
Hamiltonian (6.5). The evolution equations for the covariances is obtained from those given in reference 13 by setting $a(t)=1$ and using the frequencies $\omega_{k}^{2}(\mathcal{T})=k^{2}+\mathcal{M}^{2}(\mathcal{T})$. In particular, by setting the covariance of the diagonal elements (given by equation (2.20) in [13]; see also equation (2.44) of [13),

$$
\mathcal{A}_{k}(\mathcal{T})=-i \frac{F_{k}^{*}(\mathcal{T})}{F_{k}^{*}(\mathcal{T})}
$$

we find that with the initial conditions (6.11), the conformal time density matrix is that of local equilibrium at $\mathcal{T}_{0}$ in the sense that it commutes with the conformal time Hamiltonian. However, it is straightforward to see, that the comoving time density matrix does not commute with the comoving time Hamiltonian at the initial time $t_{0}$.

An important corollary of this analysis and comparison with other initial conditions used in comoving time is that assuming initial conditions of local equilibrium in comoving time leads to divergences that depend on the initial condition as discussed at length in [13]. This dependence of the renormalization counterterms on the initial condition was also realized in ref. [28] within the context of the CTP formulation. Imposing the initial conditions corresponding to local thermal equilibrium in conformal time, we see that: i) the renormalization counterterms do not depend on the initial conditions and ii) the mode functions are identified with those corresponding to the adiabatic vacuum for large momenta. This is why we prefer the initial conditions (6.11).

For our main analysis we choose this initial temperature to be zero so that the resulting density matrix describes a pure state, which for the large momentum modes coincides with the conformal adiabatic vacuum. Such zero temperature choice seems appropriate after the exponential inflation of the universe.

\section{Particle Number:}

We write the Fourier components of the field $\chi$ and its canonical momentum $\Pi_{\chi}$ given by $(6.20)-(6.21)$ as:

$$
\begin{aligned}
& \bar{\chi}_{k}(\mathcal{T})=\frac{1}{\sqrt{2}}\left[a_{k} F_{k}(\mathcal{T})+a_{-k}^{\dagger} F_{k}^{*}(\mathcal{T})\right], \\
& \Pi_{\chi, k}(\mathcal{T})=\frac{1}{2}\left[a_{k} F_{k}^{\prime}(\mathcal{T})+a_{-k}^{\dagger} F_{k}^{*^{\prime}}(\mathcal{T})\right] .
\end{aligned}
$$

These (conformal time) Heisenberg operators can be written equivalently in terms of the $\mathcal{T}$ dependent creation and annihilation operators

$$
\begin{aligned}
& \bar{\chi}_{k}(\mathcal{T})=\frac{1}{\sqrt{2 \omega_{k}\left(\mathcal{T}_{0}\right)}}\left[\tilde{a}_{k}(\mathcal{T}) e^{-i \omega_{k}\left(\mathcal{T}_{0}\right) \mathcal{T}}+\tilde{a}_{k}^{\dagger}(\mathcal{T}) e^{i \omega_{k}\left(\mathcal{T}_{0}\right) \mathcal{T}}\right] \\
& \Pi_{\chi, k}(\mathcal{T})=-i \sqrt{\frac{\omega_{k}\left(\mathcal{T}_{0}\right)}{2}}\left[\tilde{a}_{k}(\mathcal{T}) e^{-i \omega_{k}\left(\mathcal{T}_{0}\right) \mathcal{T}}-\tilde{a}_{k}^{\dagger}(\mathcal{T}) e^{i \omega_{k}\left(\mathcal{T}_{0}\right) \mathcal{T}}\right]
\end{aligned}
$$

The operators $\tilde{a}_{k}(\mathcal{T}) ; a_{k}(\mathcal{T})$ are related by a Bogoliubov transformation. The number of particles referred to the initial Fock vacuum of the modes $F_{k}$, is given by

$$
N_{k}(\mathcal{T})=\left\langle\tilde{a}_{k}^{\dagger}(\mathcal{T}) \tilde{a}_{k}(\mathcal{T})\right\rangle=\frac{1}{4}\left|\frac{F_{k}(\mathcal{T})}{F_{k}(0)}\right|^{2}\left[1+\frac{1}{\omega_{k}^{2}\left(\mathcal{T}_{0}\right)}\left|\frac{F_{k}^{\prime}(\mathcal{T})}{F_{k}(\mathcal{T})}\right|^{2}\right]-\frac{1}{2}
$$


or alternatively, in terms of the comoving mode functions $f_{k}(t)=F_{k}(\mathcal{T}) / C(\mathcal{T})$ we find

$$
N_{k}(t)=\frac{a^{2}(t)}{4}\left|\frac{f_{k}(t)}{f_{k}(0)}\right|^{2}\left[1+\frac{1}{\omega_{k}^{2}(0)}\left|\frac{\dot{f}_{k}(t)+H f_{k}(t)}{f_{k}(t)}\right|^{2}\right]-\frac{1}{2} .
$$

Using the large $k$-expansion of the conformal mode functions given by eqs. (6.23) we find the large- $k$ behavior of the particle number to be $N_{k} \stackrel{k \rightarrow \infty}{=} \mathcal{O}\left(1 / k^{4}\right)$, and the total number of particles (with reference to the initial state at $\mathcal{T}_{0}$ ) is therefore finite.

\section{FIELDS EVOLUTION ON A FIXED FRW BACKGROUND}

We consider in this section the matter evolution on radiation or matter dominated FRW cosmologies [14. The case for de Sitter expansion will be discussed in sec. VIII [15].

We write the scale factor as $a(t)=\left(t / t_{0}\right)^{n}$ with $n=1 / 2$ and $n=2 / 3$ corresponding to radiation and matter dominated backgrounds, respectively. Note that the value of $t_{0}$ determines the initial Hubble constant since

$$
H\left(t_{0}\right)=\frac{\dot{a}\left(t_{0}\right)}{a\left(t_{0}\right)}=\frac{n}{t_{0}} .
$$

We now solve the system of equations (4.27) - (4.29) in the Hartree approximation, with (4.11) replacing (4.27) in the large $N$ limit. We begin by presenting an early time analysis of the slow roll scenario. We then undertake a thorough numerical investigation of various cases of interest. For the symmetry broken case, we also provide an investigation of the late time behavior of the zero mode and the quantum fluctuations. We use the dimensionless variables (5.15)-(5.16).

We will assume minimal coupling to the curvature, $\xi_{r}=0$. In the cases of interest, $\mathcal{R} \ll \mu^{2}$, so that finite $\xi_{r}$ has little effect.

\section{A. Early Time Solutions for Slow Roll}

For early times in a slow roll scenario $\left[m^{2}=-\mu^{2}, \eta\left(t_{0}\right) \ll 1\right]$, we can neglect in eqs.(4.27) or (4.11) and in eq.(4.28) both the quadratic and cubic terms in $\eta(t)$ as well as the quantum fluctuations $\left\langle\psi^{2}(t)\right\rangle_{r}$ [recall that $\left.\left\langle\psi^{2}\left(t_{0}\right)\right\rangle_{r}=0\right]$. Thus, the differential equations for the zero mode (4.27) or (4.11) and the mode functions (4.28) become linear equations. In terms of the scaled variables introduced above, with $a(t)=t^{n}(n=2 / 3$ for a matter dominated cosmology while $n=1 / 2$ for a radiation dominated cosmology) we have:

$$
\begin{aligned}
\ddot{\eta}(t)+\frac{3 n}{t} \dot{\eta}(t)-\eta(t) & =0, \\
{\left[\frac{d^{2}}{d t^{2}}+\frac{3 n}{t} \frac{d}{d t}+\frac{k^{2}}{t^{2 n}}-1\right] U_{k}(t) } & =0 .
\end{aligned}
$$

The solutions to the zero mode equation $(7.1)$ are 


$$
\eta(t)=c t^{-\nu} I_{\nu}(t)+d t^{-\nu} K_{\nu}(t)
$$

where $\nu \equiv(3 n-1) / 2$, and $I_{\nu}(t)$ and $K_{\nu}(t)$ are modified Bessel functions. The coefficients, $c$ and $d$, are determined by the initial conditions on $\eta$. For $\eta\left(t_{0}\right)=\eta_{0}$ and $\dot{\eta}\left(t_{0}\right)=0$, we have:

$$
\begin{aligned}
& c=\eta_{0} t_{0}^{\nu+1}\left[\dot{K}_{\nu}\left(t_{0}\right)-\frac{\nu}{t_{0}} K_{\nu}\left(t_{0}\right)\right], \\
& d=-\eta_{0} t_{0}^{\nu+1}\left[\dot{I}_{\nu}\left(t_{0}\right)-\frac{\nu}{t_{0}} I_{\nu}\left(t_{0}\right)\right] .
\end{aligned}
$$

Taking the asymptotic forms of the modified Bessel functions, we find that for intermediate times $\eta(t)$ grows as

$$
\eta(t) \stackrel{t \gg 1}{=} \frac{c}{\sqrt{2 \pi}} t^{-3 n / 2} e^{t}\left[1-\frac{9 n^{2}-6 n}{8 t}+\mathcal{O}\left(\frac{1}{t^{2}}\right)\right] .
$$

We see that $\eta(t)$ grows very quickly in time, and the approximations (7.1) and (7.2) will quickly break down. For the case shown in fig.1 (with $n=2 / 3, \eta\left(t_{0}\right)=10^{-7}$, and $\dot{\eta}\left(t_{0}\right)=0$ ), we find that this approximation is valid up to $t-t_{0} \simeq 10$.

The equations for the mode functions $(7.2)$ can be solved in closed form for the modes in the case of a radiation dominated cosmology with $n=1 / 2$. The solutions are

$$
U_{k}(t)=c_{k} e^{-t} U\left(\frac{3}{4}-\frac{k^{2}}{2}, \frac{3}{2}, 2 t\right)+d_{k} e^{-t} M\left(\frac{3}{4}-\frac{k^{2}}{2}, \frac{3}{2}, 2 t\right) .
$$

Here, $U(\cdot)$ and $M(\cdot)$ are confluent hypergeometric functions [32] (in another common notation, $\left.M(\cdot) \equiv{ }_{1} F_{1}(\cdot)\right)$, and the $c_{k}$ and $d_{k}$ are coefficients determined by the initial conditions (4.30) on the modes. The solutions can also be written in terms of parabolic cylinder functions.

For large $t$ we have the asymptotic form

$U_{k}(t) \stackrel{t \gg 1}{=} d_{k} e^{t}(2 t)^{-\left(3 / 4+k^{2} t_{0} / 2\right)} \frac{\sqrt{\pi}}{2 \Gamma\left(\frac{3}{4}-\frac{k^{2} t_{0}}{2}\right)}\left[1+\mathcal{O}\left(\frac{1}{t}\right)\right]+c_{k} e^{-t}(2 t)^{\left(-3 / 4+k^{2} t_{0} / 2\right)}\left[1+\mathcal{O}\left(\frac{1}{t}\right)\right]$.

Again, these expressions only apply for intermediate times before the nonlinearities have grown significantly.

\section{B. Numerical Analysis}

We now present the numerical analysis of the dynamical evolution of scalar fields in time dependent, matter and radiation dominated cosmological backgrounds. We use initial values of the Hubble constant such that $H\left(t_{0}\right) \geq 0.1$. For expansion rates much less than this value the evolution will look similar to Minkowski space, which has been studied in great detail elsewhere [10,[1]. As will be seen, the equation of state found numerically is, in the majority of cases, that of cold matter. We therefore use matter dominated expansion for the evolution in much of the analysis that follows. While it presents some inconsistency at late times, the 
evolution in radiation dominated universes remains largely unchanged, although there is greater initial growth of quantum fluctuations due to the scale factor growing more slowly in time. Using the large $N$ and Hartree approximations to study theories with continuous and discrete symmetries respectively, we treat three important cases. They are 1) $m^{2}<0$, $\left.\left.\eta\left(t_{0}\right) \ll 1 ; 2\right) m^{2}<0, \eta\left(t_{0}\right) \gg 1 ; 3\right) m^{2}>0, \eta\left(t_{0}\right) \gg 1$.

In presenting the figures, we have shifted the origin of time such that $t \rightarrow t^{\prime}=t-t_{0}$. This places the initial time, $t_{0}$, at the origin. In these shifted coordinates, the scale factor is given by

$$
a(t)=\left(\frac{t+\tau}{\tau}\right)^{n}
$$

where, once again, $n=2 / 3$ and $n=1 / 2$ in matter and radiation dominated backgrounds respectively, and the value of $\tau$ is determined by the Hubble constant at the initial time:

$$
H\left(t_{0}=0\right)=\frac{n}{\tau}
$$

Case 1: $m^{2}<0, \eta\left(t_{0}\right) \ll 1$. This is the case of an early universe phase transition in which there is little or no biasing in the initial configuration (by biasing we mean that the initial conditions break the $\eta \rightarrow-\eta$ symmetry). The transition occurs from an initial temperature above the critical temperature, $T>T_{c}$, which is quenched at $t_{0}$ to the temperature $T_{f} \ll T_{c}$. This change in temperature due to the rapid expansion of the universe is modeled here by an instantaneous change in the mass from an initial value $m_{i}^{2}=T^{2} / T_{c}^{2}-1$ to a final value $m_{f}^{2}=-1$. We will use the value $m_{i}^{2}=1$ in what follows. This quench approximation is necessary since the low momentum frequencies (4.16) appearing in our initial conditions (4.30) are complex for negative mass squared and small $\eta\left(t_{0}\right)$. An alternative choice is to use initial frequencies given by

$$
\omega_{k}\left(t_{0}\right)=\left[k^{2}+\mathcal{M}^{2}\left(t_{0}\right) \tanh \left(\frac{k^{2}+\mathcal{M}^{2}\left(t_{0}\right)}{\left|\mathcal{M}^{2}\left(t_{0}\right)\right|}\right)\right]^{1 / 2} .
$$

These frequencies have the attractive feature that they match the conformal adiabatic frequencies given by (4.16) for large values of $k$ while remaining positive for small $k$. We find that such a choice of initial conditions changes the quantitative value of the particle number by a few percent, but leaves the qualitative results unchanged.

We plot the the zero mode $\eta(t)$, the equal time correlator $g \Sigma(t)$, the total number of produced particles $g N(t)$ (see sec. VI for a discussion of our definition of particles), the number of particles $g N_{k}(t)$ as a function of wavenumber for both intermediate and late times, and the ratio of the pressure and energy densities $p(t) / \varepsilon(t)$ (giving the equation of state).

Figs. 1a-e shows these quantities in the large $N$ approximation for a matter dominated cosmology with an initial condition on the zero mode given by $\eta\left(t_{0}=0\right)=10^{-7}, \dot{\eta}\left(t_{0}=0\right)=0$ and for an initial expansion rate of $H\left(t_{0}\right)=0.1$. This choice for the initial value of $\eta$ stems from the fact that the quantum fluctuations only have time to grow significantly for initial values satisfying $\eta\left(t_{0}\right) \ll \sqrt{g}$; for values $\eta\left(t_{0}\right) \gg \sqrt{g}$ the evolution is essentially classical. This result is clear from the intermediate time dependence of the zero mode and the low momentum mode functions given by the expressions (7.6) and (7.8) respectively. 


\section{FIGURES}

Figure 1a
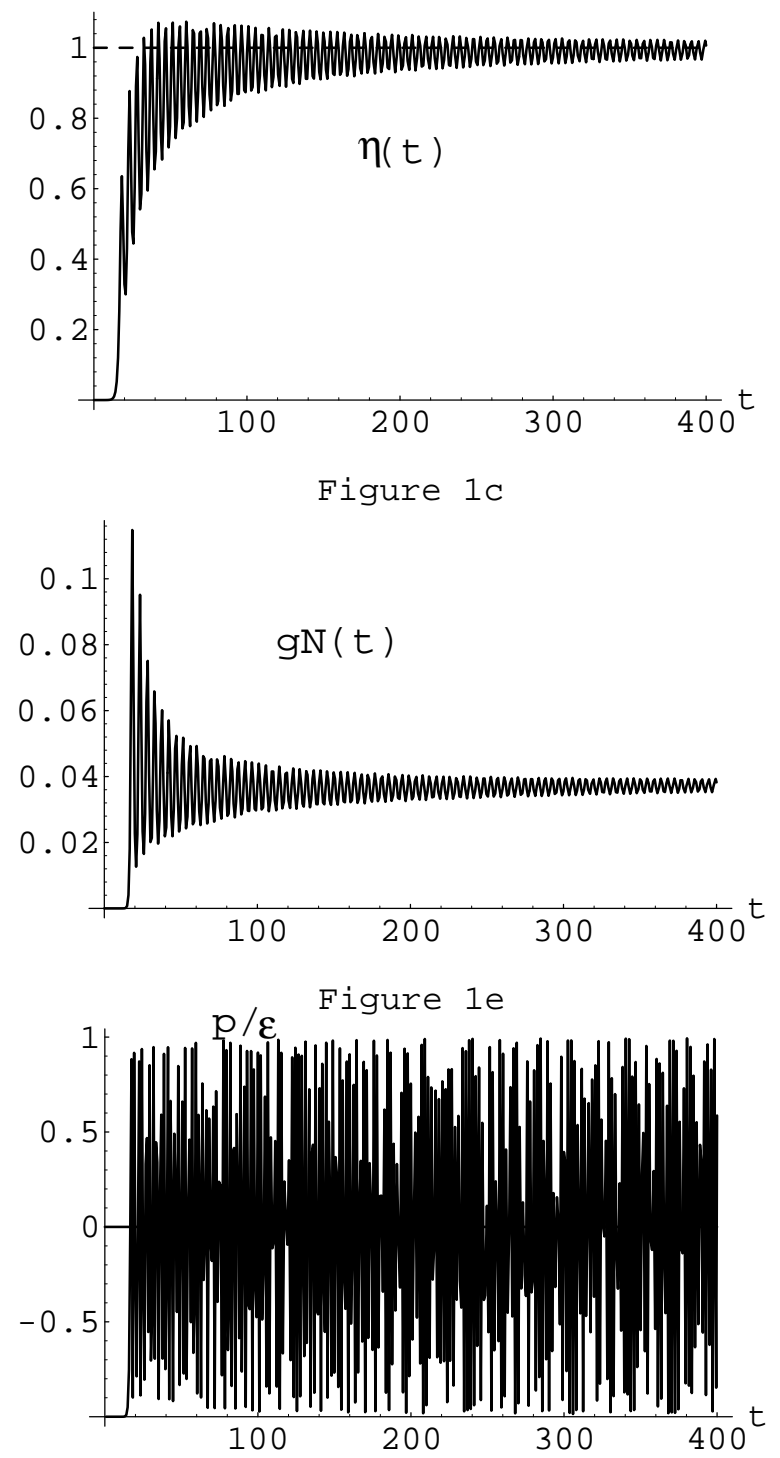

Figure 1b

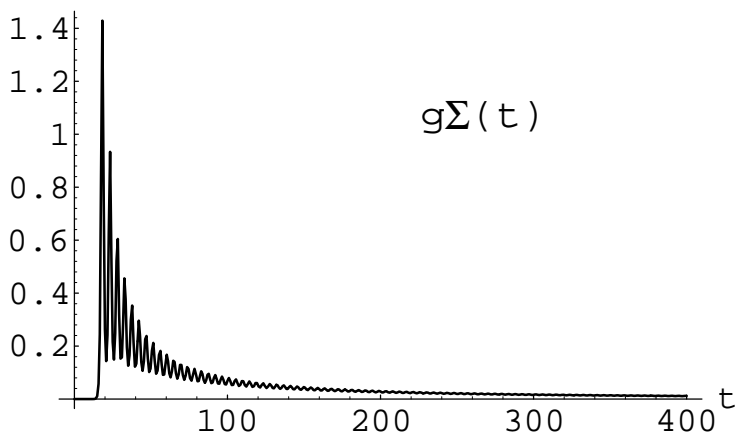

Figure 1d

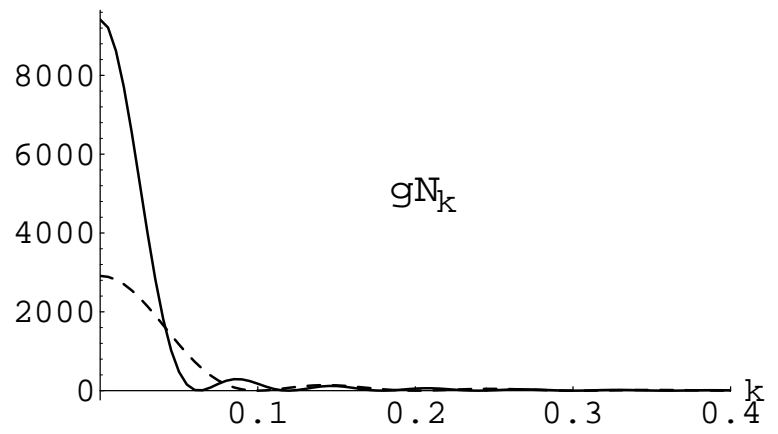

FIG. 1. Symmetry broken, slow roll, large $N$, matter dominated evolution of (a) the zero mode $\eta(t)$ vs. $t$, (b) the quantum fluctuation operator $g \Sigma(t)$ vs. $t$, (c) the number of particles $g N(t)$ vs. $t,(\mathrm{~d})$ the particle distribution $g N_{k}(t)$ vs. $k$ at $t=149.1$ (dashed line) and $t=398.2$ (solid line), and (e) the ratio of the pressure and energy density $p(t) / \varepsilon(t)$ vs. $t$ for the parameter values $m^{2}=-1, \eta\left(t_{0}\right)=10^{-7}, \dot{\eta}\left(t_{0}\right)=0, g=10^{-12}, H\left(t_{0}\right)=0.1$.

After the initial growth of the fluctuation $g \Sigma(t)$ (fig.1b) we see that the zero mode (fig.1a) approaches the value given by the minimum of the tree level potential, $\eta=1$, while $g \Sigma(t)$ decays for late times as

$$
g \Sigma(t) \simeq \frac{\mathcal{C}}{a^{2}(t)}=\frac{\mathcal{C}}{t^{4 / 3}}
$$

For these late times, the Ward identity corresponding to the $O(N)$ symmetry of the field 
theory is satisfied, enforcing the condition

$$
-1+\eta^{2}(t)+g \Sigma(t)=0 .
$$

Hence, the zero mode approaches the classical minimum as

$$
\eta^{2}(t) \simeq 1-\frac{\mathcal{C}}{a^{2}(t)} .
$$

Figure 1c depicts the number of particles produced. After an initial burst of particle production, the number of particles settles down to a relatively constant value. Notice that the number of particles produced is approximately of order $1 / \mathrm{g}$. In fig.1d, we show the number of particles as a function of the wavenumber, $k$. For intermediate times we see the simple structure depicted by the dashed line in the figure, while for late times this quantity becomes concentrated more at low values of the momentum $k$.

Finally, fig.1e shows that the field begins with a de Sitter equation of state $p=-\varepsilon$ but evolves quickly to a state dominated by ordinary matter, with an equation of state (averaged over the oscillation timescale) $p=0$. This last result is a bit surprising as one expects from the condition (7.9) that the particles produced in the final state are massless Goldstone bosons which should have the equation of state of radiation. However, as shown in fig.1d, the produced particles are of low momentum, $q \ll 1$, and while the effective mass of the particles is zero to very high accuracy when averaged over the oscillation timescale, the effective mass makes small oscillations about zero so that the dispersion relation for these particles differs from that of radiation. In addition, since the produced particles have little energy, the contribution to the energy density from the zero mode, which contributes to a cold matter equation of state, remains significant.

In figs. 2a-e we show the same situation depicted in fig. 1 using the Hartree approximation. The initial condition on the zero mode is $\eta\left(t_{0}=0\right)=\sqrt{3} \cdot 10^{-7}$; the factor of $\sqrt{3}$ appears due to the different scaling in the zero mode equations, (4.27) and (5.18), which causes the minimum of the tree level effective potential in the Hartree approximation to have a value of $\eta=\sqrt{3}$. Again, the Hubble constant has the value $H\left(t_{0}\right)=0.1$. Here, we see again that there is an initial burst of particle production as $g \Sigma(t)$ (fig.2b) grows large. However, the zero mode (fig.2a) quickly reaches the minimum of the potential and the condition

$$
-1+\eta^{2}(t) / 3+g \Sigma(t)=0
$$

is approximately satisfied by forcing the value of $g \Sigma(t)$ quickly to zero.

There are somewhat fewer particles produced here compared to the large $N$ case, and the distribution of particles is more extended. Since the effective mass of the particles is nonzero, we expect a matter dominated equation of state (fig 2e) for later times. The fact that the Hartree approximation does not satisfy Goldstone's theorem means that the resulting particles must be massive, explaining why somewhat fewer particles are produced.

Finally, we show the special case in which there is no initial biasing in the field, $\eta\left(t_{0}=\right.$ $0)=0, \dot{\eta}\left(t_{0}=0\right)=0$, and $H\left(t_{0}\right)=0.1$ in figs. 3a-d. With such an initial condition, the Hartree approximation and the large $N$ limit are equivalent. The zero mode remains zero for all time, so that the quantity $g \Sigma(t)$ (fig.3a) satisfies the sum rule (7.9) by reaching the value one without decaying for late times. Notice that many more particles are produced in 
this case (fig $3 b$ ); the growth of the particle number for late times is due to the expansion of the universe. The particle distribution (fig.3c) is similar to that of the slow roll case in fig.1. The equation of state (fig.3d) is likewise similar.

Figure $2 a$
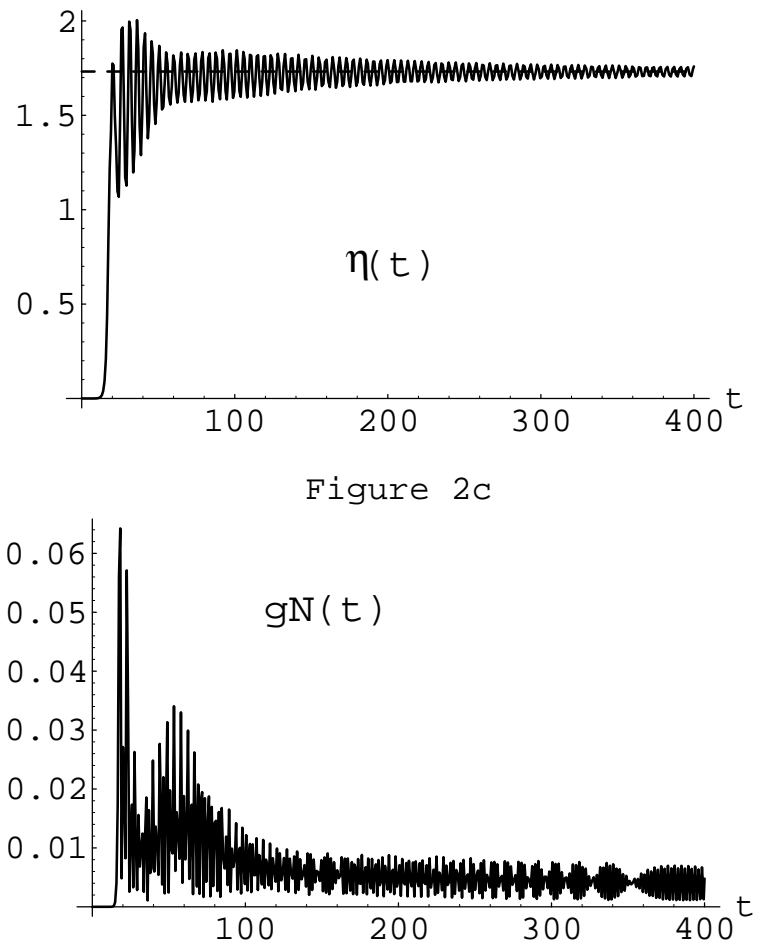

$\mathrm{p} / \varepsilon$ Figure $2 \mathrm{e}$

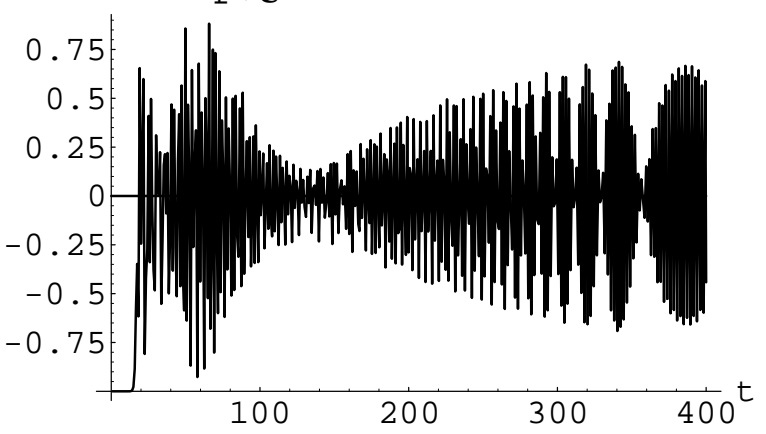

Figure 2b

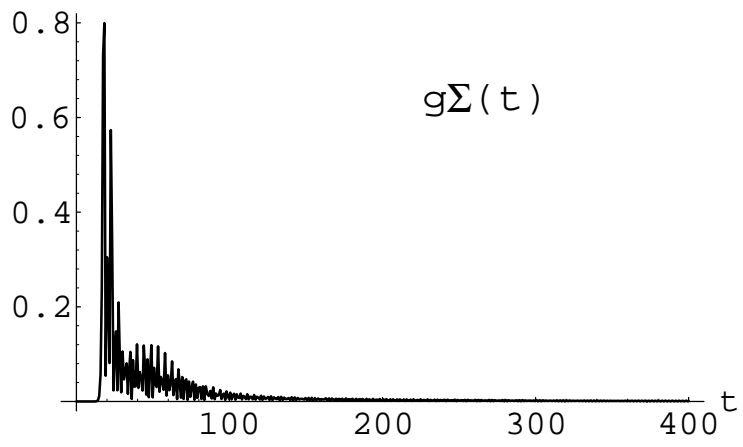

Figure $2 d$

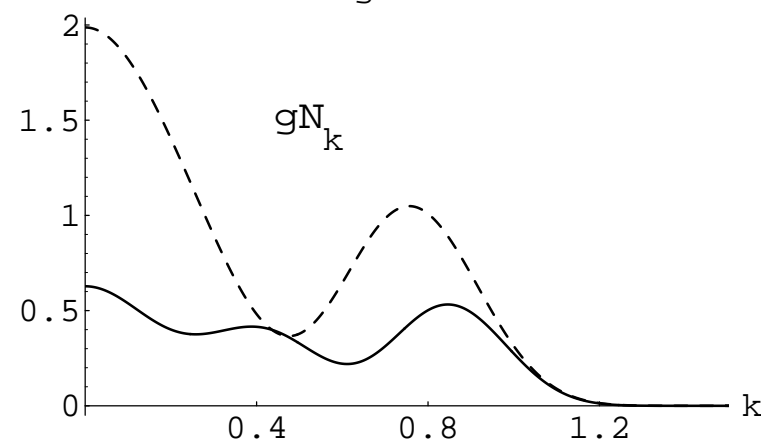

FIG. 2. Symmetry broken, slow roll, Hartree, matter dominated evolution of (a) the zero mode $\eta(t)$ vs. $t$, (b) the quantum fluctuation operator $g \Sigma(t)$ vs. $t,(\mathrm{c})$ the number of particles $g N(t)$ vs. $t,(\mathrm{~d})$ the particle distribution $g N_{k}(t)$ vs. $k$ at $t=150.7$ (dashed line) and $t=396.1$ (solid line), and (e) the ratio of the pressure and energy density $p(t) / \varepsilon(t)$ vs. $t$ for the parameter values $m^{2}=-1, \eta\left(t_{0}\right)=3^{1 / 2} \cdot 10^{-7}, \dot{\eta}\left(t_{0}\right)=0, g=10^{-12}, H\left(t_{0}\right)=0.1$.

In each of these cases of slow roll dynamics, increasing the Hubble constant has the effect of slowing the growth of both $\eta$ and $g \Sigma(t)$. The equation of state will be that of a de Sitter universe for a longer period before moving to a matter dominated equation of state. 
Otherwise, the dynamics is much the same as in figs. 1-3.

Case 2: $m^{2}<0, \eta\left(t_{0}\right) \gg 1$. We now examine the case of a chaotic inflationary scenario with a symmetry broken potential. In chaotic inflation, the zero mode begins with a value $\eta(t) \gg 1$. During the de Sitter phase, $H \gg 1$, and the field initially evolves classically, dominated by the first order derivative term appearing in the zero mode equation (see (4.27) and (4.28)). Eventually, the zero mode rolls down the potential, ending the de Sitter phase and beginning the FRW phase. We consider the field dynamics in the FRW universe after the end of inflation. We thus take the initial temperature to be zero, $T=0$.
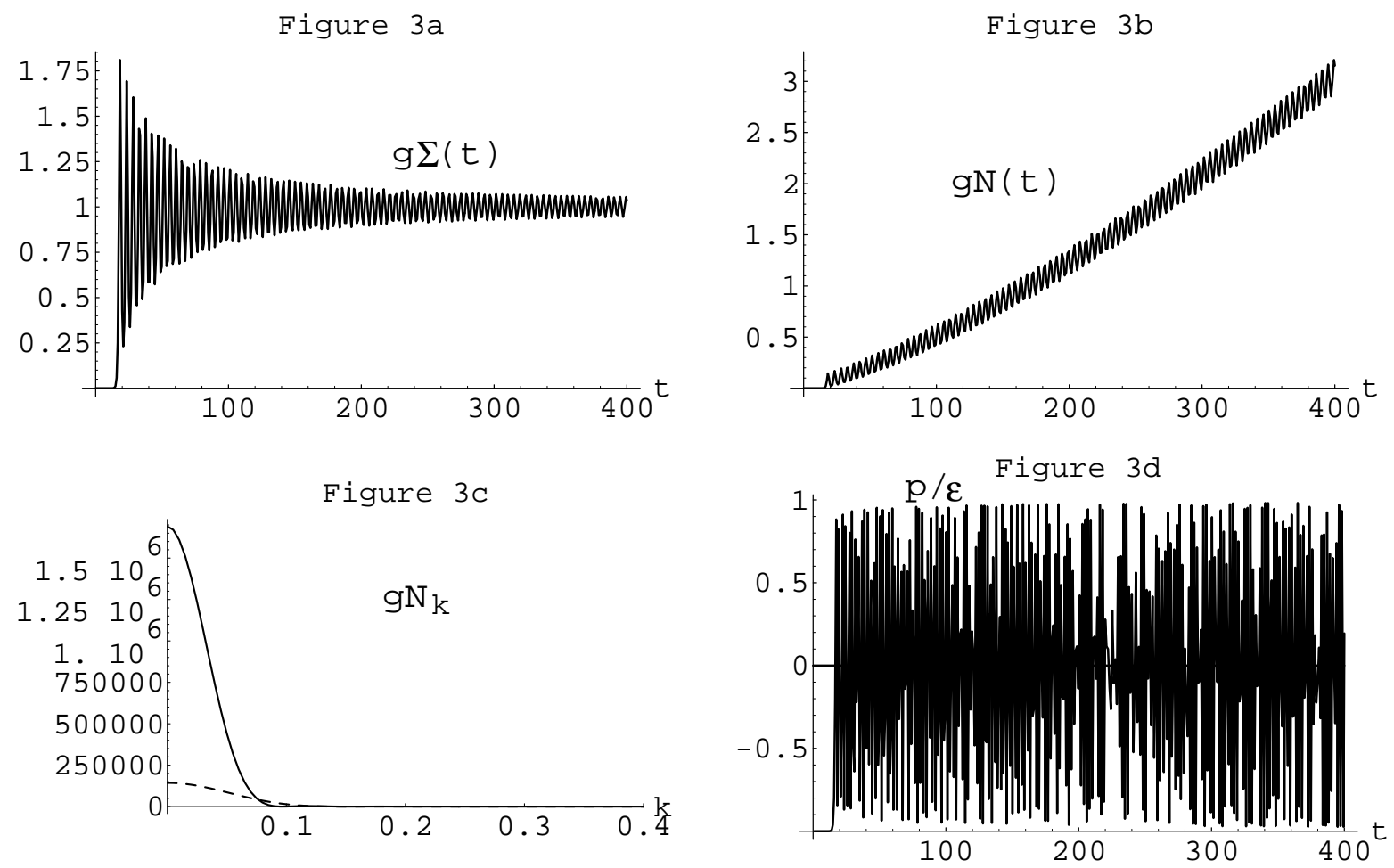

FIG. 3. Symmetry broken, no roll, matter dominated evolution of (a) the quantum fluctuation operator $g \Sigma(t)$ vs. $t$, (b) the number of particles $g N(t)$ vs. $t,(\mathrm{c})$ the particle distribution $g N_{k}(t)$ vs. $k$ at $t=150.1$ (dashed line) and $t=397.1$ (solid line), and (d) the ratio of the pressure and energy density $p(t) / \varepsilon(t)$ vs. $t$ for the parameter values $m^{2}=-1, \eta\left(t_{0}\right)=0, \dot{\eta}\left(t_{0}\right)=0, g=10^{-12}$, $H\left(t_{0}\right)=0.1$.

Figure 4 shows our results for the quantities, $\eta(t), g \Sigma(t), g N(t), g N_{k}(t)$, and $p(t) / \varepsilon(t)$ for the evolution in the large $N$ approximation within a radiation dominated gravitational background with $H\left(t_{0}\right)=0.1$. The initial condition on the zero mode is chosen to have the representative value $\eta\left(t_{0}=0\right)=4$ with $\dot{\eta}\left(t_{0}=0\right)=0$. Initial values of the zero mode much smaller than this will not produce significant growth of quantum fluctuations; initial values larger than this produces qualitatively similar results, although the resulting number of particles will be greater and the time it takes for the zero mode to settle into its asymptotic state will be longer. 

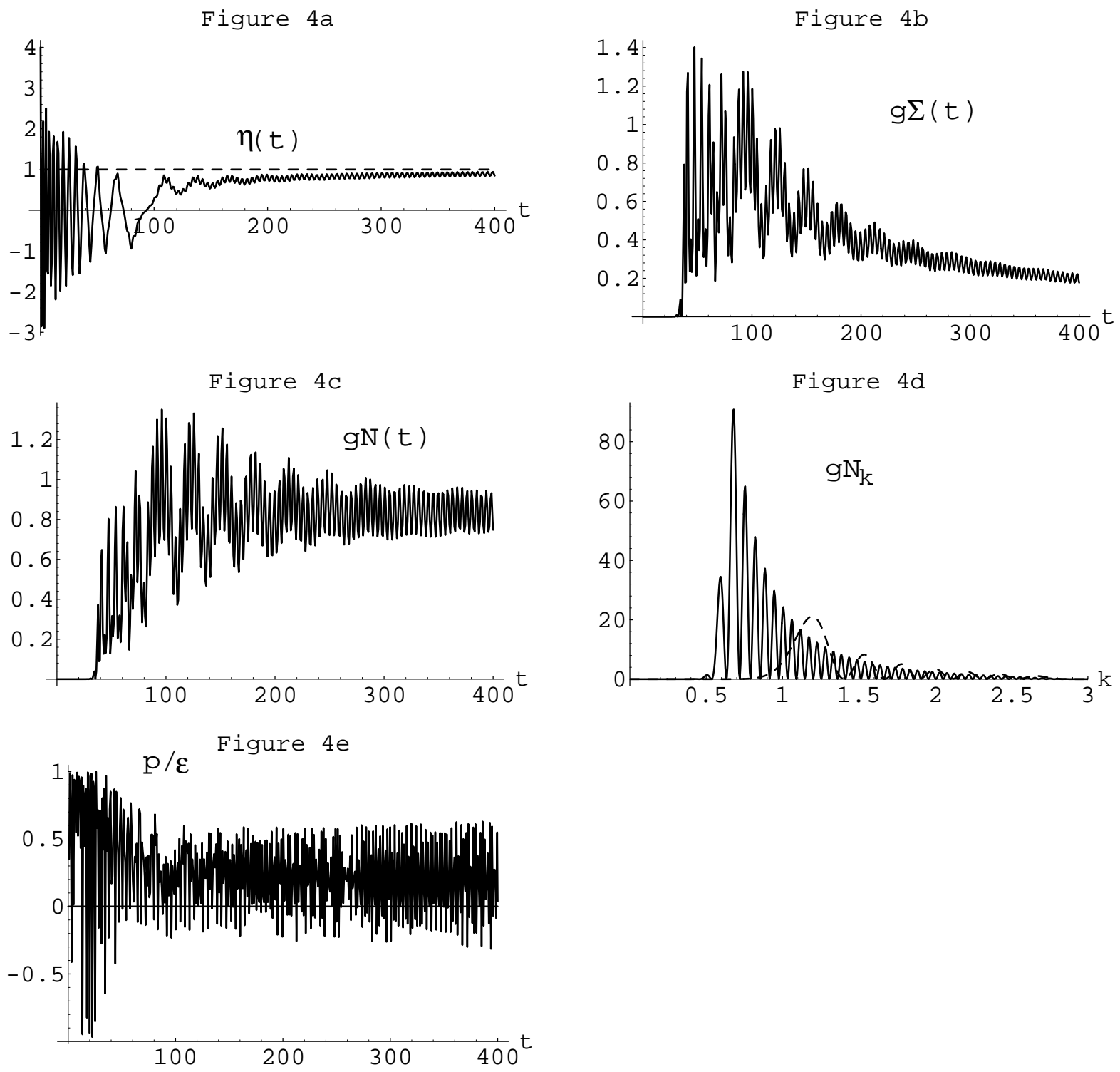

FIG. 4. Symmetry broken, chaotic, large $N$, radiation dominated evolution of (a) the zero mode $\eta(t)$ vs. $t,(\mathrm{~b})$ the quantum fluctuation operator $g \Sigma(t)$ vs. $t,(\mathrm{c})$ the number of particles $g N(t)$ vs. $t$, (d) the particle distribution $g N_{k}(t)$ vs. $k$ at $t=76.4$ (dashed line) and $t=392.8$ (solid line), and (e) the ratio of the pressure and energy density $p(t) / \varepsilon(t)$ vs. $t$ for the parameter values $m^{2}=-1$, $\eta\left(t_{0}\right)=4, \dot{\eta}\left(t_{0}\right)=0, g=10^{-12}, H\left(t_{0}\right)=0.1$.

We see from fig.4a that the zero mode oscillates rapidly, while the amplitude of the oscillation decreases due to the expansion of the universe. This oscillation induces particle production through the process of parametric amplification (fig.4c) and causes the fluctuation $g \Sigma(t)$ to grow (fig.4b). Eventually, the zero mode loses enough energy that it is restricted to one of the two minima of the tree level effective potential. The subsequent evolution closely follows that of Case 1 above with $g \Sigma(t)$ decaying in time as $1 / a^{2}(t) \sim 1 / t$ with $\eta$ given by the sum rule (7.9). The spectrum (fig.4d) indicates a single unstable band 
of particle production dominated by the modes $k=1 / 2$ to about $k=3$ for late times. The structure within this band becomes more complex with time and shifts somewhat toward lower momentum modes. Such a shift is also observed in Minkowski spacetimes [10 12]. Figure $4 \mathrm{e}$ shows the equation of state which we see to be somewhere between the relations for matter and radiation for times out as far as $t=400$, but slowly moving to a matter equation of state. Since matter redshifts as $1 / a^{3}(t)$ while radiation redshifts as $1 / a^{4}(t)$, the equation of state should eventually become matter dominated. Given the equation of state indicated by fig.4e, we estimate that this occurs for times of order $t=10^{4}$. The reason the equation of state in this case differs from that of cold matter as was seen in figs. 1-3 is that the particle distribution produced by parametric amplification is concentrated at higher momenta, $k \simeq 1$.

Figure 5 shows the corresponding case with a matter dominated background. The results are qualitatively very similar to those described for fig. 4 above. Due to the faster expansion, the zero mode (fig.5a) finds one of the two wells more quickly and slightly less particles are produced. For late times, the fluctuation $g \Sigma(t)$ (fig.5b) decays as $1 / a^{2}(t) \propto 1 / t^{4 / 3}$. Again we see an equation of state (figs. 5e) which evolves from a state between that of pure radiation or matter toward one of cold matter.

The Hartree case is depicted in fig.6 for a matter dominated universe, with the initial condition on the zero mode $\eta\left(t_{0}=0\right)=4 \sqrt{3}$. Again, the evolution begins in much the same manner as in the large $N$ approximation with oscillation of the zero mode (fig.6a), which eventually settles into one of the two minima of the effective potential. Whereas in the large $N$ approximation, the zero mode approaches the minimum asymptotically [as given by (7.9) and our late time analysis below], in the Hartree approximation we see that the zero mode finds the minimum quickly and proceeds to oscillate about that value. The two point correlator (fig.6b) quickly evolves toward zero without growing large. Particle production in the Hartree approximation (figs. 6c-d) is again seen to be inefficient compared to that of the large $N$ case above. Fig. 6e again shows that the equation of state is matter dominated for all but the earliest times.

A larger Hubble constant prevents significant particle production unless the initial amplitude of the zero mode is likewise increased such that the relation $\eta\left(t_{0}\right) \gg H\left(t_{0}\right)$ is satisfied. For very large amplitude $\eta\left(t_{0}\right) \gg 1$, to the extent that the mass term can be neglected and while the quantum fluctuation term has not grown to be large, the equations of motion (4.27), (4.28), and (4.11) are scale invariant with the scaling $\eta \rightarrow \mu \eta, H \rightarrow \mu H, t \rightarrow t / \mu$, and $k \rightarrow \mu k$, where $\mu$ is an arbitrary scale.

Case 3: $m^{2}>0, \eta\left(t_{0}\right) \gg 1$. The final case we examine is that of a simple chaotic scenario with a positive mass term in the Lagrangian. Again, the FRW stage occurs after the inflationary expansion; this allows us to take zero initial temperature.

Figure 7 shows this situation in the large $N$ approximation for a matter dominated cosmology. The zero mode, $\eta(t)$, oscillates in time while decaying in amplitude from its initial value of $\eta\left(t_{0}=0\right)=5, \dot{\eta}\left(t_{0}=0\right)=0$ (fig.7a), while the quantum fluctuation, $g \Sigma(t)$, grows rapidly for early times due to parametric resonance (figs. 7b). We choose here an initial condition on the zero mode which differs from that of figs 4-5 above since there is no significant growth of quantum fluctuations for smaller initial values. From fig.7d, we see 
Figure $5 \mathrm{a}$

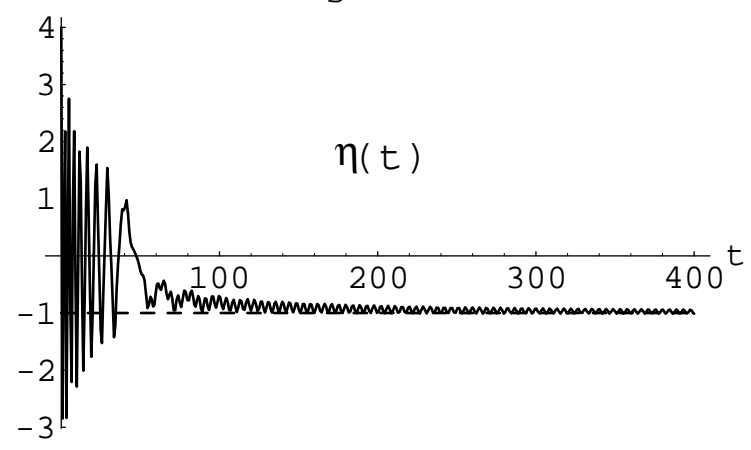

Figure 5c
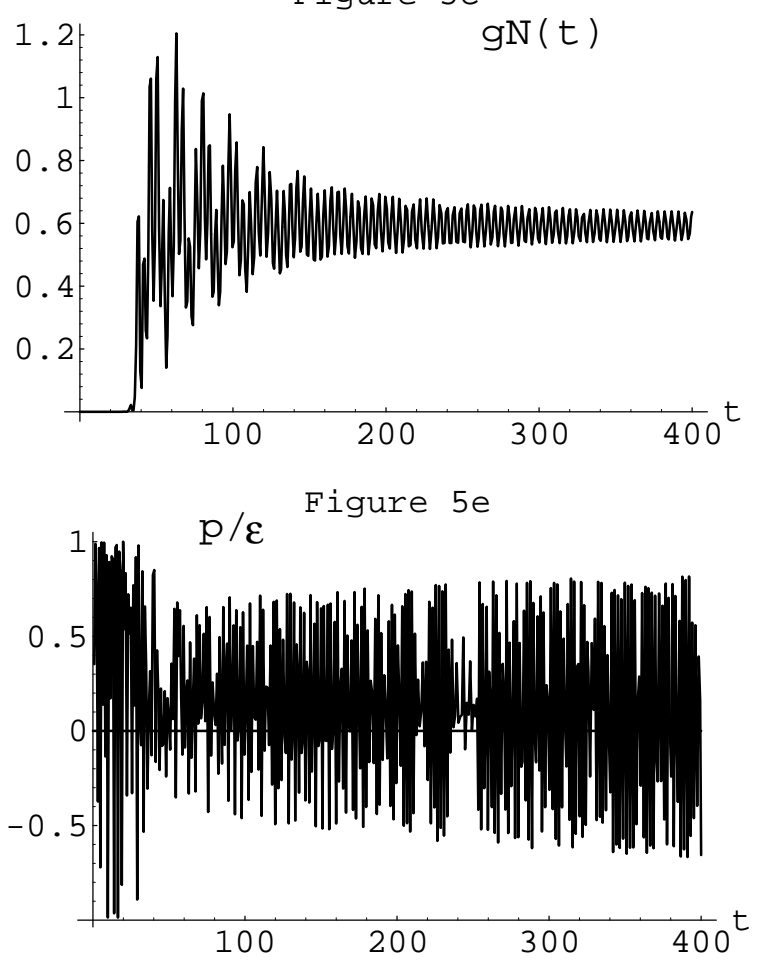
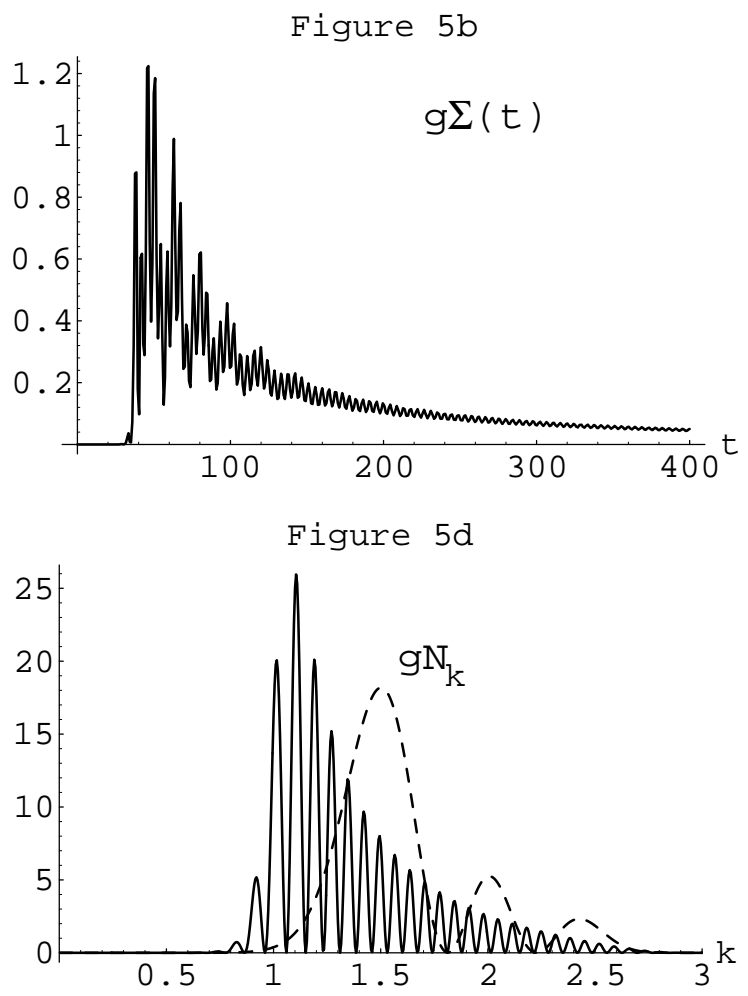

FIG. 5. Symmetry broken, chaotic, large $N$, matter dominated evolution of (a) the zero mode $\eta(t)$ vs. $t$, (b) the quantum fluctuation operator $g \Sigma(t)$ vs. $t,(\mathrm{c})$ the number of particles $g N(t)$ vs. $t$, (d) the particle distribution $g N_{k}(t)$ vs. $k$ at $t=50.8$ (dashed line) and $t=399.4$ (solid line), and (e) the ratio of the pressure and energy density $p(t) / \varepsilon(t)$ vs. $t$ for the parameter values $m^{2}=-1$, $\eta\left(t_{0}\right)=4, \dot{\eta}\left(t_{0}\right)=0, g=10^{-12}, H\left(t_{0}\right)=0.1$.

that there exists a single unstable band at values of roughly $k=1$ to $k=3$, although careful examination reveals that the unstable band extends all the way to $k=0$. The equation of state is depicted by the quantity $p(t) / \varepsilon(t)$ in fig.7e. As expected in this massive theory, the equation of state is matter dominated. 

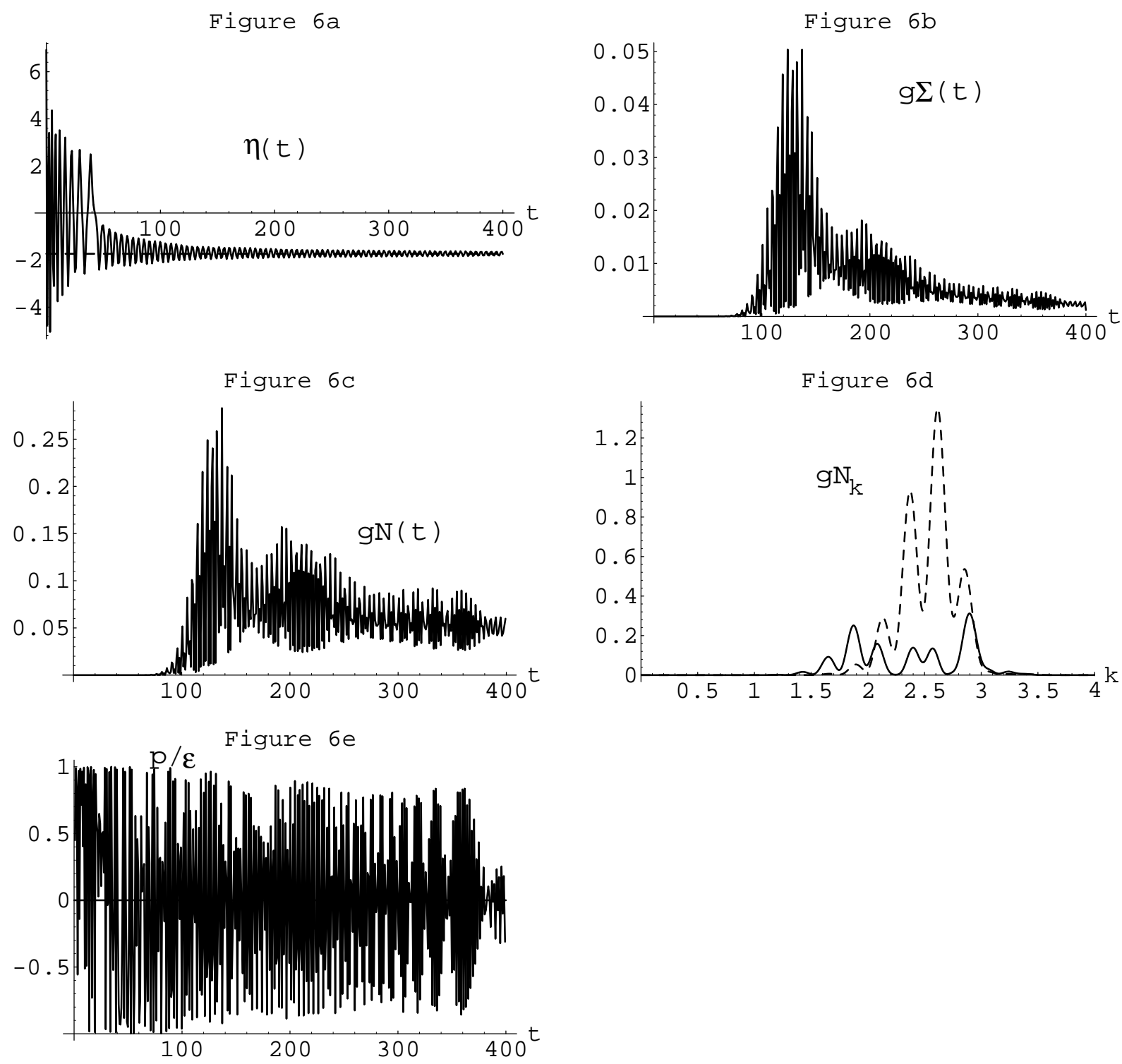

FIG. 6. Symmetry broken, chaotic, Hartree, matter dominated evolution of (a) the zero mode $\eta(t)$ vs. $t$, (b) the quantum fluctuation operator $g \Sigma(t)$ vs. $t$, (c) the number of particles $g N(t)$ vs. $t,(\mathrm{~d})$ the particle distribution $g N_{k}(t)$ vs. $k$ at $t=151.3$ (dashed line) and $t=397.0$ (solid line), and (e) the ratio of the pressure and energy density $p(t) / \varepsilon(t)$ vs. $t$ for the parameter values $m^{2}=-1, \eta\left(t_{0}\right)=4 \cdot 3^{1 / 2}, \dot{\eta}\left(t_{0}\right)=0, g=10^{-12}, H\left(t_{0}\right)=0.1$.

The final case is the Hartree approximation, shown in fig.8. Here, parametric amplification is entirely inefficient when expansion of the universe is included and we require an initial condition on the zero mode of $\eta\left(t_{0}=0\right)=12 \sqrt{3}$ to provide even meager growth of quantum fluctuations. We have used a matter dominated gravitational background with $H\left(t_{0}\right)=0.1$. We see that while the zero mode oscillates (fig.8a), there is little growth in quantum fluctuations (fig.8b) and few particles produced (fig.8c). Examining the particle distribution (fig.8d), it is found that the bulk of these particles is produced within a single 

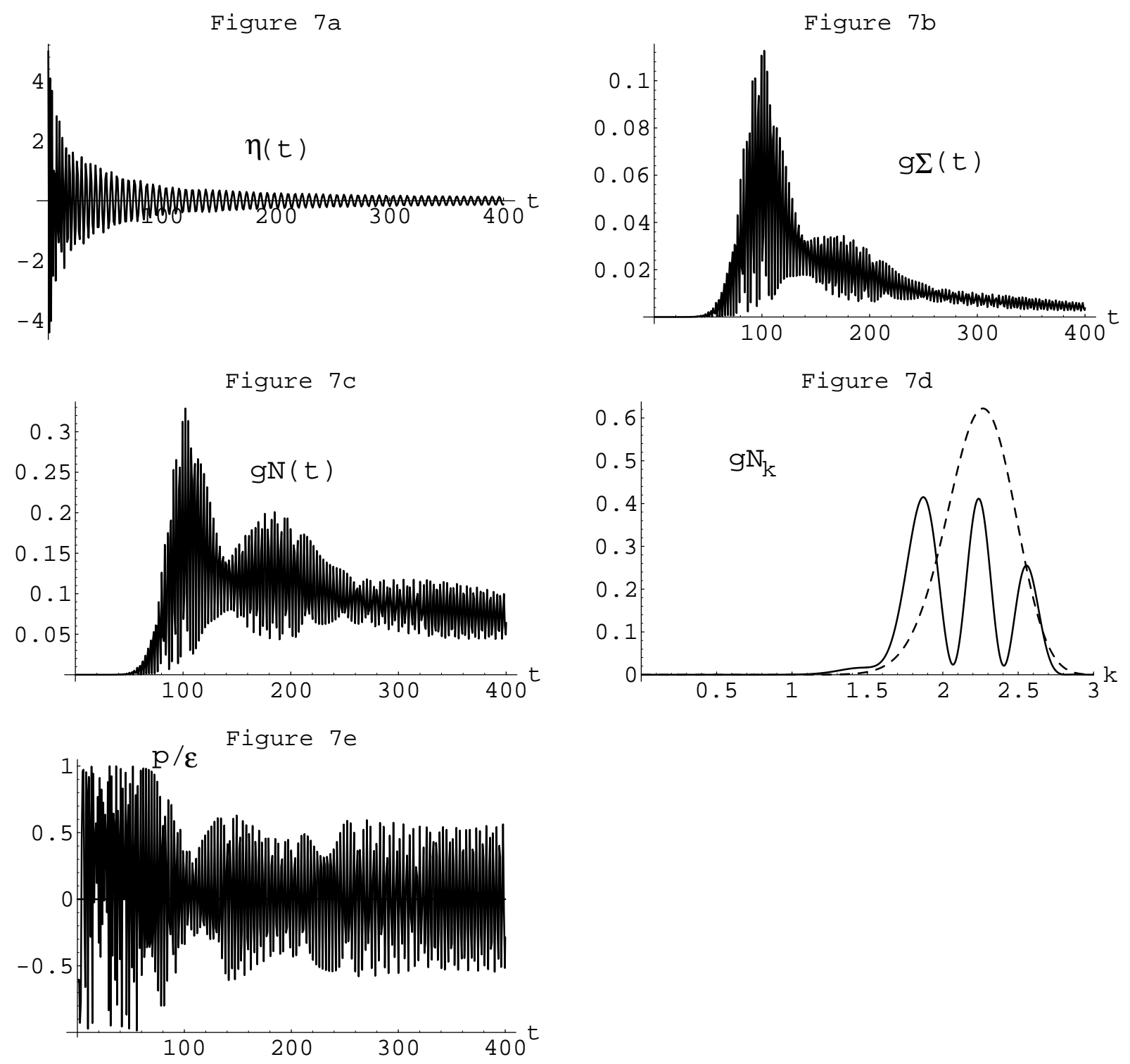

FIG. 7. Symmetry unbroken, chaotic, large $N$, matter dominated evolution of (a) the zero mode $\eta(t)$ vs. $t$, (b) the quantum fluctuation operator $g \Sigma(t)$ vs. $t$, (c) the number of particles $g N(t)$ vs. $t,(\mathrm{~d})$ the particle distribution $g N_{k}(t)$ vs. $k$ at $t=77.4$ (dashed line) and $t=399.7$ (solid line), and (e) the ratio of the pressure and energy density $p(t) / \varepsilon(t)$ vs. $t$ for the parameter values $m^{2}=+1, \eta\left(t_{0}\right)=5, \dot{\eta}\left(t_{0}\right)=0, g=10^{-12}, H\left(t_{0}\right)=0.1$.

resonance band extending from $k \simeq 15$ to $k \simeq 16$. This resonance develops at early time during the large amplitude oscillation of the zero mode. These results are explained by a simple resonance band analysis described below.

At first glance, it is not entirely clear why there are so many more particles produced in the large $N$ case of fig.7 than in the Hartree case of fig.8. Since in the present case the Hubble time is long compared to the oscillation timescale of the zero mode, $H \ll 1$, we would expect a forbidden band for early times at the location given approximately by the 

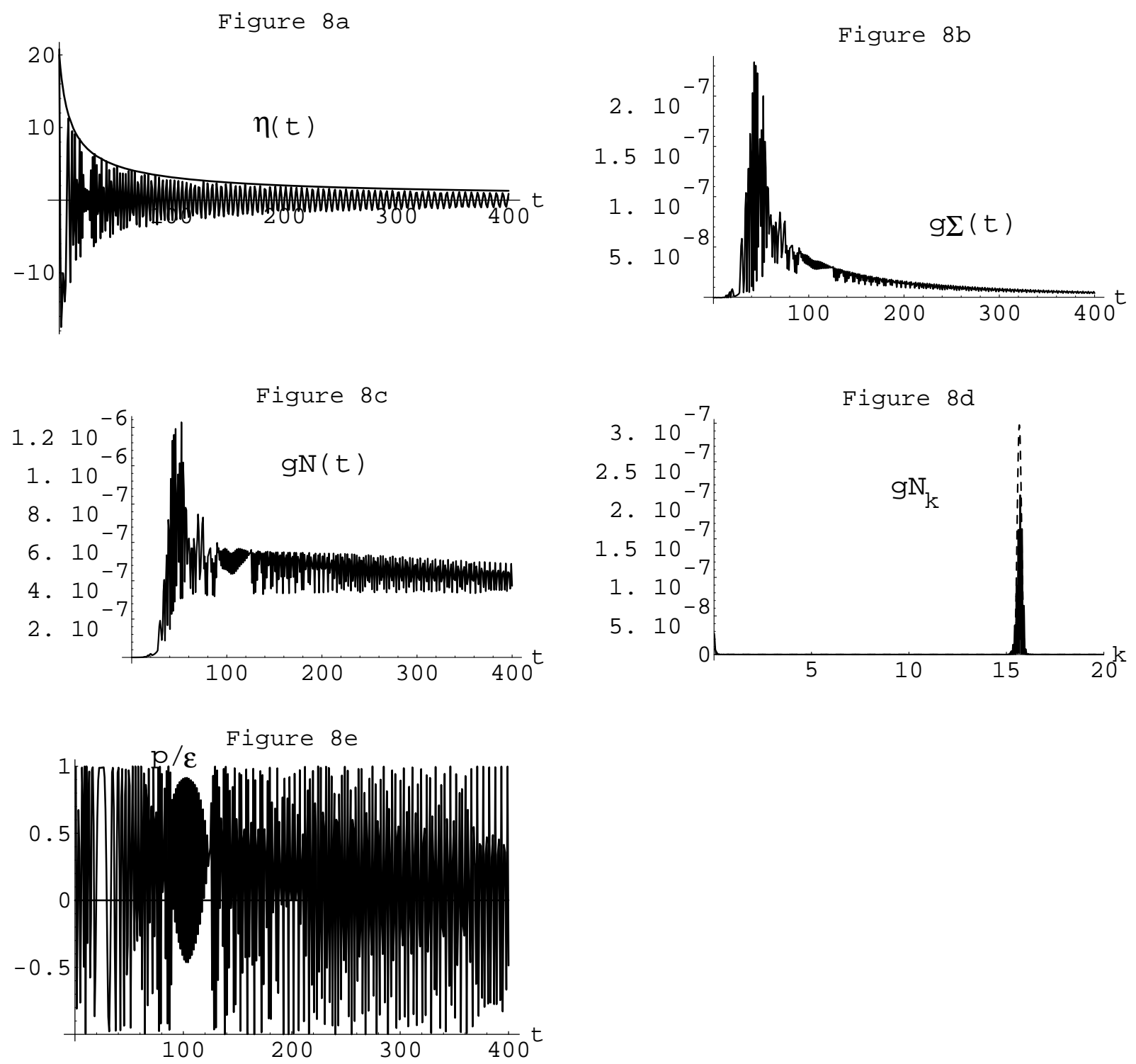

FIG. 8. Symmetry unbroken, chaotic, Hartree, matter dominated evolution of (a) the zero mode $\eta(t)$ vs. $t$, (b) the quantum fluctuation operator $g \Sigma(t)$ vs. $t$, (c) the number of particles $g N(t)$ vs. $t,(\mathrm{~d})$ the particle distribution $g N_{k}(t)$ vs. $k$ at $t=50.5$ (dashed line) and $t=391.2$ (solid line), and (e) the ratio of the pressure and energy density $p(t) / \varepsilon(t)$ vs. $t$ for the parameter values $m^{2}=+1, \eta\left(t_{0}\right)=12 \cdot 3^{1 / 2}, \dot{\eta}\left(t_{0}\right)=0, g=10^{-12}, H\left(t_{0}\right)=0.1$.

Minkowski results provided in Ref. [11]. In fact, we find this to be the case.

The solution to this problem is inherent in the band structure of the two cases when combined with an understanding of the dynamics in an expanding spacetime. First, we note that, for early times when $g \Sigma(t) \ll 1$, the zero mode is well fit by the function $\eta(t)=$ $\eta_{0} f(t) / a(t)$ where $f(t)$ is an oscillatory function taking on values from -1 to 1 . This is clearly seen from the envelope function $\eta_{0} / a(t)$ shown in fig.8a (recall that $g \Sigma(t) \ll 1$ during the entire evolution in this case). Second, the momentum that appears in the equations 
for the modes (4.28) is the physical momentum $k / a(t)$. We therefore write the approximate expressions for the locations of the forbidden bands in FRW by using the Minkowski results of [11] with the substitutions $\eta_{0}^{2} \rightarrow \gamma \eta_{0}^{2} / a^{2}(t)$ (where the factor of $\gamma$ accounts for the difference in the definition of the non-linear coupling between this study and [11]) and $q^{2} \rightarrow k^{2} / a^{2}(t)$.

Making these substitutions, we find for the location in comoving momentum $k$ of the forbidden band in the large $N$ (fig.7) and Hartree (fig.8) cases:

$$
\begin{aligned}
0 & \leq k^{2} \leq \frac{\eta_{0}^{2}}{2}, \quad(\operatorname{large} N) \\
\frac{\eta_{0}^{2}}{2}+3 a^{2}(t) & \leq k^{2} \leq a^{2}(t)\left(\sqrt{\frac{\eta_{0}^{4}}{3 a^{4}(t)}+\frac{2 \eta_{0}^{2}}{a^{2}(t)}+4}+1\right), \quad \text { (Hartree) } .
\end{aligned}
$$

The important feature to notice is that while the location of the unstable band (to a first approximation) in the case of the continuous $O(N)$ theory is the same as in Minkowski and does not change in time, the location of the band is time dependent in the discrete theory described by the non-perturbative Hartree approximation.

While $\eta_{0} / a(t) \gg 1$, the Hartree relation reduces to

$$
\frac{\eta_{0}^{2}}{2} \leq k^{2} \leq \frac{\eta_{0}^{2}}{\sqrt{3}}
$$

This is the same as the Minkowski result for large amplitude, and one finds that this expression accurately predicts the location of the resonance band of fig.8d. However, with time the band shifts its location toward higher values of comoving momentum as given by (7.12), cutting off particle production in that initial band. There is continuing particle production for higher modes, but since the Floquet index is decreased due to the reduced amplitude of the zero mode, since there is no enhancement of production of particles in these modes (as these modes begin with at most of order 1 particles), and because the band continues to shift to higher momenta while becoming smaller in width, this particle production never becomes significant.

As in the symmetry broken case of figs. 4-6, the equations of motion for large amplitude and relatively early times are approximately scale invariant. In fig.9 we show the case of the large $N$ evolution in a radiation dominated universe with initial Hubble constant of $H\left(t_{0}\right)=2$ with appropriately scaled initial value of the zero mode of $\eta\left(t_{0}\right)=16$. Again, the qualitative dynamics remains largely unchanged from the case of a smaller Hubble constant.

\section{Late Time Behavior}

We see clearly from the numerical evolution that in the case of a symmetry broken potential, the late time large $N$ solutions obey the sum rule (5.20). This sum rule is a consequence of the late time Ward identities which enforce Goldstone's Theorem. Because

of this sum rule, we can write down the analytical expressions for the late time behavior of the fluctuations and the zero mode. Using (5.20), the mode equation (4.28) becomes

$$
\left[\frac{d^{2}}{d t^{2}}+3 \frac{\dot{a}(t)}{a(t)} \frac{d}{d t}+\frac{k^{2}}{a^{2}(t)}\right] U_{k}(t)=0 .
$$


This equation can be solved exactly if we assume a power law dependence for the scale factor
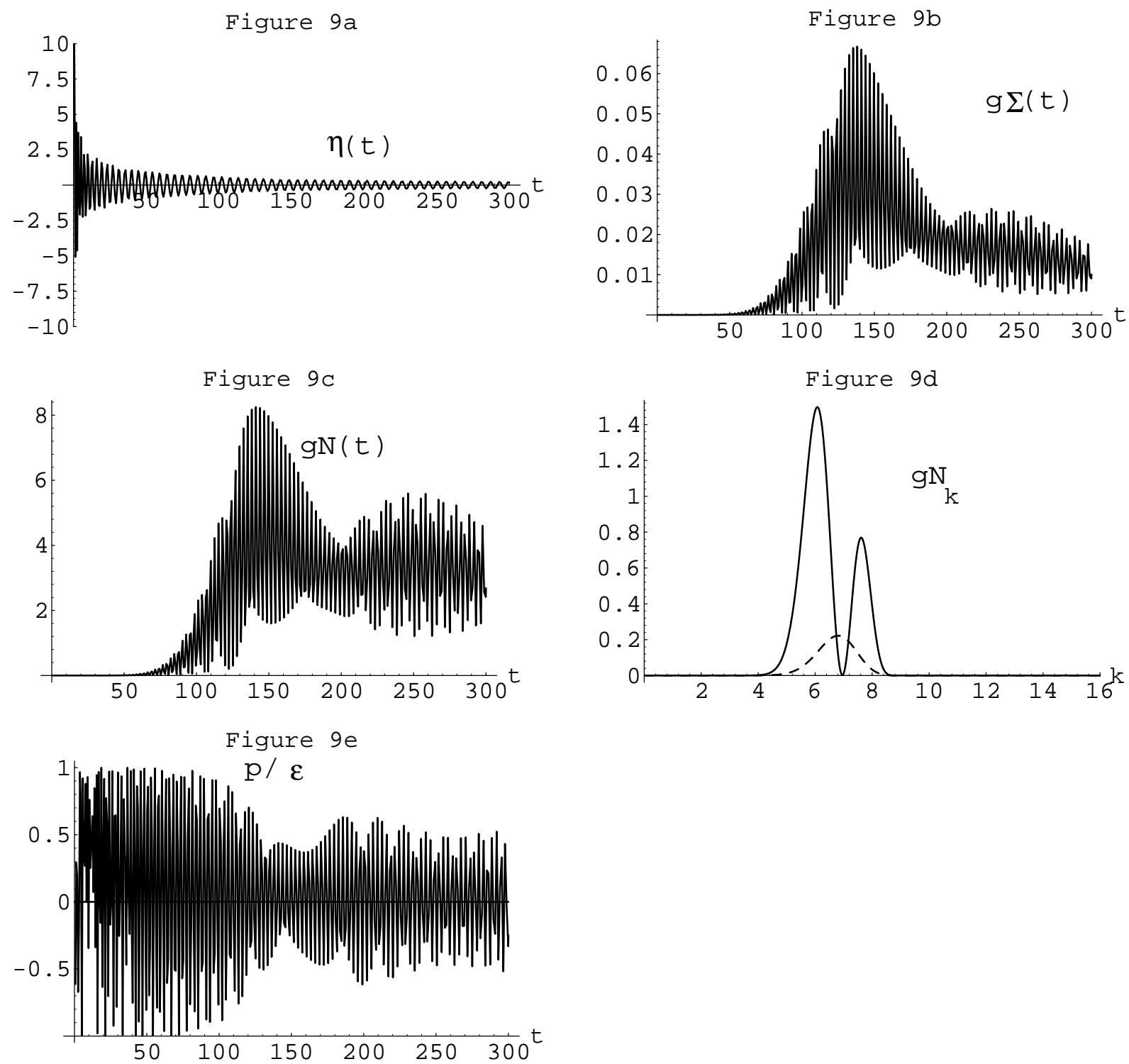

FIG. 9. Symmetry unbroken, chaotic, large $N$, radiation dominated evolution of (a) the zero mode $\eta(t)$ vs. $t$, (b) the quantum fluctuation operator $g \Sigma(t)$ vs. $t$, (c) the number of particles $g N(t)$ vs. $t,(\mathrm{~d})$ the particle distribution $g N_{k}(t)$ vs. $k$ at $t=102.1$ (dashed line) and $t=251.6$ (solid line), and (e) the ratio of the pressure and energy density $p(t) / \varepsilon(t)$ vs. $t$ for the parameter values $m^{2}=+1, \eta\left(t_{0}\right)=16, \dot{\eta}\left(t_{0}\right)=0, g=10^{-12}, H\left(t_{0}\right)=2.0$.

$$
\begin{aligned}
& a(t)=\left(t / t_{0}\right)^{n} \text {. The solution is } \\
& \quad U_{k}(t)=c_{k} t^{(1-3 n) / 2} J_{\frac{1-3 n}{2-2 n}}\left(\frac{k t_{0}^{n} t^{1-n}}{n-1}\right)+d_{k} t^{(1-3 n) / 2} Y_{\frac{1-3 n}{2-2 n}}\left(\frac{k t_{0}^{n} t^{1-n}}{n-1}\right),
\end{aligned}
$$

where $J_{\nu}$ and $Y_{\nu}$ are Bessel and Neumann functions respectively, and the constants $c_{k}$ and $d_{k}$ carry dependence on the initial conditions and on the dynamics up to the point at which 
the sum rule is satisfied.

These functions have several important properties. In particular, in radiation or matter dominated universes, $n<1$, and for values of wavenumber satisfying $k \gg t^{-(1-n)} / t_{0}^{n}$, the mode functions decay in time as $1 / a(t) \sim t^{-n}$. Since the sum rule applies for late times, $t-t_{0} \gg 1$ in dimensionless units, we see that all values of $k$ except a very small band about $k=0$ redshift as $1 / a(t)$. The $k=0$ mode, however, remains constant in time, explaining the support evidenced in the numerical results for values of small $k$ (see figs. 1,3). These results mean that the quantum fluctuation has a late time dependence of $\left\langle\psi^{2}(t)\right\rangle_{r} \sim 1 / a^{2}(t)$. The late time dependence of the zero mode is given by this expression combined with the sum rule (5.20). These results are accurately reproduced by our numerical analysis. Note that qualitatively this late time dependence is independent of the choice of initial conditions for the zero mode, except that there is no growth of modes near $k=0$ in the case in which particles are produced via parametric amplification (figs. 4,5).

For the radiation $n=\frac{1}{2}$ and matter dominated $n=\frac{2}{3}$ universes, eq.(7.15) reduces to elementary functions:

$$
\begin{aligned}
& a(t) U_{k}(t)=c_{k} e^{2 i k t_{0}^{1 / 2} t^{1 / 2}}+d_{k} e^{-2 i k t_{0}^{1 / 2} t^{1 / 2}}(\mathrm{RD}), \\
& a(t) U_{k}(t)=c_{k} e^{3 i k t_{0}^{2 / 3} t^{1 / 3}}\left[1+\frac{i}{3 k t_{0}^{2 / 3} t^{1 / 3}}\right]+d_{k} e^{-3 i k t_{0}^{2 / 3} t^{1 / 3}}\left[1-\frac{i}{3 k t_{0}^{2 / 3} t^{1 / 3}}\right] \quad(\mathrm{MD}) .
\end{aligned}
$$

It is also of interest to examine the $n>1$ case. Here, the modes of interest satisfy the condition $k \ll t^{n-1} / t_{0}^{n}$ for late times. These modes are constant in time and one sees that the modes are frozen. In the case of a de Sitter universe, we can formally take the limit

$n \rightarrow \infty$ and we see that all modes become frozen at late times. This case is detailed in sec. VIII [15].

\section{Discussion and Conclusions for the FRW background}

We have shown that there can be significant particle production through quantum fluctuations after inflation [14]. However, this production is somewhat sensitive to the expansion of the universe. From our analysis of the equation of state, we see that the late time dynamics is given by a matter dominated cosmology. We have also shown that the quantum fluctuations of the inflaton decay for late times as $1 / a^{2}(t)$, while in the case of a symmetry broken inflationary model, the inflaton field moves to the minimum of its tree level potential. The exception to this behavior is the case when the inflaton begins exactly at the unstable extremum of its potential for which the fluctuations grow out to the minimum of the potential and do not decay. Initial production of particles due to parametric amplification is significantly greater in chaotic scenarios with symmetry broken potentials than in the corresponding theories with positive mass terms in the Lagrangian, given similar initial conditions on the zero mode of the inflaton. 


\section{FIELDS EVOLUTION ON A FIXED INFLATIONARY BACKGROUND (THE DE SITTER UNIVERSE)}

We describe in this section the matter evolution on a fixed de Sitter background using large $N$ and Hartree approaches. The evolution with a dynamical background is treated in sec. IX.

\section{A. Evolution for $\phi(0)=\dot{\phi}(0)=0$. Analytical Results}

We begin by considering the broken symmetry situation in which the expectation value of the inflaton field sits atop the potential hill with zero initial velocity. This situation is expected to arise if the system is initially in local thermodynamic equilibrium an initial temperature larger than the critical temperature and cools down through the critical temperature in the absence of an external field or bias.

The order parameter and its time derivative vanish in the local equilibrium high temperature phase, and this condition is a fixed point of the evolution equation for the zero mode of the inflaton. There is no rolling of the inflaton zero mode in this case, although the fluctuations will grow and will be responsible for the dynamics.

We can understand the early stages of the dynamics analytically as follows. For very weak coupling and early time we can neglect the backreation in the mode equations, which in both the large $N$ and Hartree cases become,

$$
\begin{gathered}
{\left[\frac{d^{2}}{d \tau^{2}}+3 h \frac{d}{d \tau}+\frac{q^{2}}{a^{2}(\tau)}-1\right] f_{q}(\tau)=0} \\
f_{q}(0)=\frac{1}{\sqrt{\omega_{q}}} ; \quad \dot{f}_{q}(\tau)=-i \sqrt{\omega_{q}} ; \quad \omega_{q}=\sqrt{q^{2}+r^{2}-1} .
\end{gathered}
$$

The solutions are of the form,

$$
f_{q}(\tau)=\exp \left[-\frac{3}{2} h \tau\right]\left\{a(q) J_{\nu}(z)+b(q) J_{-\nu}(z)\right\} ; z=\frac{q}{h} \exp [-h \tau] ; \nu=\sqrt{\frac{1}{h^{2}}+\frac{9}{4}}
$$

where the coefficients $a(q)$ and $b(q)$ are determined by the initial conditions:

$$
\begin{aligned}
& b(q)=-\frac{\pi q}{2 h \sin \nu \pi}\left[\frac{i \omega_{q}-\frac{3}{2} h}{q} J_{\nu}\left(\frac{q}{h}\right)-J_{\nu}^{\prime}\left(\frac{q}{h}\right)\right], \\
& a(q)=\frac{\pi q}{2 h \sin \nu \pi}\left[\frac{i \omega_{q}-\frac{3}{2} h}{q} J_{-\nu}\left(\frac{q}{h}\right)-J_{-\nu}^{\prime}\left(\frac{q}{h}\right)\right] .
\end{aligned}
$$

For long times, $e^{h \tau} \geq q / h$, these mode functions grow exponentially,

$$
f_{q}(\tau) \simeq b(q) J_{-\nu}(z) \simeq \frac{b(q)}{\Gamma(1-\nu)}\left(\frac{2 h}{q}\right)^{\nu} e^{(\nu-3 / 2) h \tau}
$$


The Bessel functions appearing in the expression for the modes $f_{q}(\tau)$ can be approximated by their series expansion,

$$
f_{q}(\tau)=\frac{1}{2}\left[1+\frac{1}{\nu}\left(\frac{3}{2}-\frac{q^{2}}{4 h^{2}}-i \frac{\omega_{q}}{h}\right)+\mathcal{O}\left(\frac{1}{\nu^{2}}\right)\right] e^{(\nu-3 / 2) h \tau} .
$$

This is an expansion in powers of $q^{2} /\left(\nu h^{2}\right)$ and we conclude that $g \Sigma(\tau)$ is dominated by the modes with $q \leq \sqrt{h}$.

The integral for $g \Sigma(\tau)$ can be approximated by keeping only the modes $q \leq f \sqrt{h}$, where $f$ is a number of order one, and by neglecting the subtraction term which will cancel the contributions from high momenta. Numerically, even with the backreaction taken into account, the integral is dominated by modes $q \leq f \approx 10-20$ in all of the cases that we studied (see fig.12 below).

The contribution to the fluctuations from these unstable modes is:

$$
g \Sigma(\tau) \simeq \sqrt{\frac{g}{3}} \frac{f^{3} h^{3 / 2} r m_{R}^{2}}{2 \pi M_{0}^{2}}\left(1+\frac{M_{0}^{2}}{m_{R}^{2}}\right) e^{(2 \nu-3) h \tau},
$$

where again, we have taken the high temperature limit, $T_{i} \sim T_{c} \gg m_{R}$.

From this equation, we can estimate the value of $\tau_{s}$, the 'spinodal time', at which the contribution of the quantum fluctuations becomes comparable to the contribution from the tree level terms in the equations of motion. This time scale is obtained from the condition $g \Sigma\left(\tau_{s}\right)=\mathcal{O}(1)$

$$
\tau_{s} \simeq-\frac{1}{(2 \nu-3) h} \ln \left[\sqrt{\frac{g}{3}} \frac{f^{3} h^{3 / 2}}{2 \pi m_{R} M_{0}^{2}} \frac{T_{i}}{T_{c}}\left(1+\frac{M_{0}^{2}}{m_{R}^{2}}\right)\right],
$$

which is in good agreement with our numerical results, as will become clear below (see figs. 10, 15 and 18). For values of $h \geq 1$, which, as argued below, lead to the most interesting case, an estimate for the spinodal time is,

$$
\tau_{s} \simeq \frac{3 h}{2} \ln [1 / \sqrt{g}]+\mathcal{O}(1)
$$

which is consistent with our numerical results (see fig.10).

For $\tau>\tau_{s}$, the effects of backreaction become very important, and the contribution from the quantum fluctuations competes with the tree level terms in the equations of motion, shutting-off the instabilities. Beyond $\tau_{s}$, only a full numerical analysis will capture the correct dynamics.

It is worth mentioning that had we chosen zero temperature initial conditions, then the coupling $\bar{g} \rightarrow g$ (see (19)) and the estimate for the spinodal time would have been,

$$
\tau_{s} \simeq \frac{3 h}{2} \ln [1 / g]+\mathcal{O}(1)
$$

that is, roughly a factor 2 larger than the estimate for which the de Sitter stage began at a temperature above the critical value. Therefore (8.10) represents an underestimate of the spinodal time scale at which fluctuations become comparable to tree level contributions. 
The number of e-folds occurring during the stage of growth of spinodal fluctuations is therefore,

$$
\mathcal{N}_{e} \approx \frac{3 h^{2}}{2} \ln [1 / \sqrt{g}]
$$

or in the zero temperature case,

$$
\mathcal{N}_{e} \approx \frac{3 h^{2}}{2} \ln [1 / g]
$$

which is a factor 2 larger. Thus, it becomes clear that with $g \approx 10^{-12}$ and $h \geq 2$, a required number of e-folds, $\mathcal{N}_{e} \approx 100$ can easily be accommodated before the fluctuations become large, modifying the dynamics and the equation of state.

The implications of these estimates are important. The first conclusion drawn from these estimates is that a 'quench' approximation is well justified (see fig.10). While the temperature drops from an initial value of a few times the critical temperature to below critical in just a few e-folds, the contribution of the quantum fluctuations needs a large number of e-folds to grow to compensate for the tree-level terms and overcome the instabilities. Only for a strongly coupled theory is the time scale for the quantum fluctuations to grow short enough to restore local thermodynamic equilibrium during the transition.

The second conclusion is that most of the growth of spinodal fluctuations occurs during the inflationary stage, and with $g \approx 10^{-12}$ and $H \geq m_{R}$, the quantum fluctuations become of the order of the tree-level contributions to the equations of motion within the number of e-folds necessary to solve the horizon and flatness problems. Since the fluctuations grow to become of the order of the tree level contributions at times of the order of this time scale, for larger times they will modify the equation of state substantially and will be shown to provide a graceful exit from the inflationary phase within an acceptable number of e-folds.

For $\tau<\tau_{s}$, when the contribution from the renormalized quantum fluctuations can be ignored, the Hubble constant is given by the classical contribution to the energy density. In terms of the dimensionless quantities introduced above (5.16), we have,

$$
H=\frac{2 m_{R}^{4}}{3 \pi g M_{p l}^{2}}\left[\frac{\dot{\eta}^{2}}{2}+\frac{1}{4}\left(\eta^{2}-1\right)^{2}\right]
$$

In the situation we consider here, with $\dot{\eta}=\eta=0$, the condition that $h \geq 2$ for $g \simeq 10^{-12}$ translates into $m_{R} \simeq 10^{13} \mathrm{GeV}$, which is an acceptable bound on the inflaton mass.

To understand more clearly whether or not the effect of quantum fluctuations and growth of unstable modes during the inflationary phase transition can provide a graceful exit scenario, we must study in detail the contribution to the energy and the equation of state of these quantum fluctuations.

Although we are working in a fixed de Sitter background, the energy and pressure will evolve dynamically. A measure of the backreaction effects of quantum fluctuations on the dynamics of the scale factor is obtained from defining the 'effective Hubble constant',

$$
\mathcal{H}^{2}(\tau)=\frac{8 \pi}{3 M_{p l}^{2}} \varepsilon(\tau)
$$


Therefore, the quantities,

$$
\frac{\mathcal{H}(\tau)}{\mathcal{H}(0)}=\sqrt{\frac{\varepsilon(\tau)}{\varepsilon(0)}} \quad \text { and } \quad \frac{\dot{\mathcal{H}}(\tau)}{\mathcal{H}^{2}(\tau)}=-\frac{3}{2}\left[1+\frac{p(\tau)}{\varepsilon(\tau)}\right]
$$

give dynamical information of the effects of the backreaction of the quantum fluctuations on the dynamics of the scale factor. Whenever $p(\tau)+\varepsilon(\tau) \neq 0, \mathcal{H}(\tau) / \mathcal{H}(0) \neq 1$, or $\dot{\mathcal{H}}(\tau) / \mathcal{H}^{2}(\tau) \neq 0$, the backreaction from the quantum fluctuations will dramatically change the dynamics of the scale factor, and it will no longer be consistent to treat the scale factor as fixed. When $\mathcal{H}(\tau) / \mathcal{H}(0) \ll 1$ the de Sitter era will end.

From this point onwards only a full treatment of the backreaction, including the correct dynamics of the scale factor, will describe the physics. This will be the subject of sec. IX |16.

\section{B. Numerical Analysis}

We now solve numerically the large $N$ set of equations (5.18) with the initial conditions (5.19), taking $\dot{\eta}(0)=\eta(0)=0$.

The numerical code is based on a fourth order Runge-Kutta algorithm for the differential equation and an 11-points Newton-Cotes algorithm for the integral, with a typical relative errors $10^{-9}$ in the differential equation and in the integrals. We have tested for cutoff insensitivity with cutoffs $q_{\max } \approx 50 ; 100 ; 150$ with no appreciable variation in the numerical results. The reason for this cutoff insensitivity is due to the fact that only long-wavelength modes grow in amplitude to become non-perturbatively large, whereas the short-wavelength modes always have perturbatively small amplitudes. We have chosen $r=T_{i} / T_{c}=2$ as a representative value and $g=10^{-12}$.

As argued previously, for $g \approx 10^{-12}$, the cosmologically interesting time scales for the spinodal instabilities to grow during say the first 60-100 e-folds of inflation occur for $h \geq 1$, leading to $H \geq m_{R} \geq 10^{13} \mathrm{GeV}$ which is a phenomenologically acceptable range for the Hubble constant during the inflationary stage.

Fig. 10 shows the contribution from the quantum fluctuations, $g \Sigma(\tau)$ vs. $\tau$ for $g=$ $10^{-12} ; T_{i} / T_{c}=2 ; h=2 ; \eta(0)=0 ; \dot{\eta}(0)=0$. The quantum fluctuations, as measured by $g \Sigma(\tau)$, grow to be of order 1 in a time scale $\tau \approx 40$ which is the time scale predicted by the early time estimate (8.10). We have checked numerically that the energy is covariantly conserved, obeying the relation $\dot{\varepsilon}+3 H(p+\varepsilon)=0$ to our numerical accuracy of one part in $10^{7}$. Fig. 11 shows $\mathcal{H}(\tau) / \mathcal{H}(0)$ vs. $\tau$. This figure shows clearly that when the spinodal quantum fluctuations become comparable to the tree level contribution to the equations of motion, the backreaction on the scale factor becomes fairly large. At this point, the approximation of keeping a fixed background breaks down and the full self-consistent dynamics will have to be studied. At this time, the inflationary stage basically ends since $\mathcal{H}$ is no longer constant. This occurs for $\tau \approx 40$ giving about 80 e-folds of inflation during the time in which $\mathcal{H}$ is approximately constant and equal to $H$. Therefore, this new mechanism of spinodal fluctuations, with the zero mode sitting atop the potential hill provides a graceful exit of the inflationary era without any further assumptions on the evolution of the scalar field. 


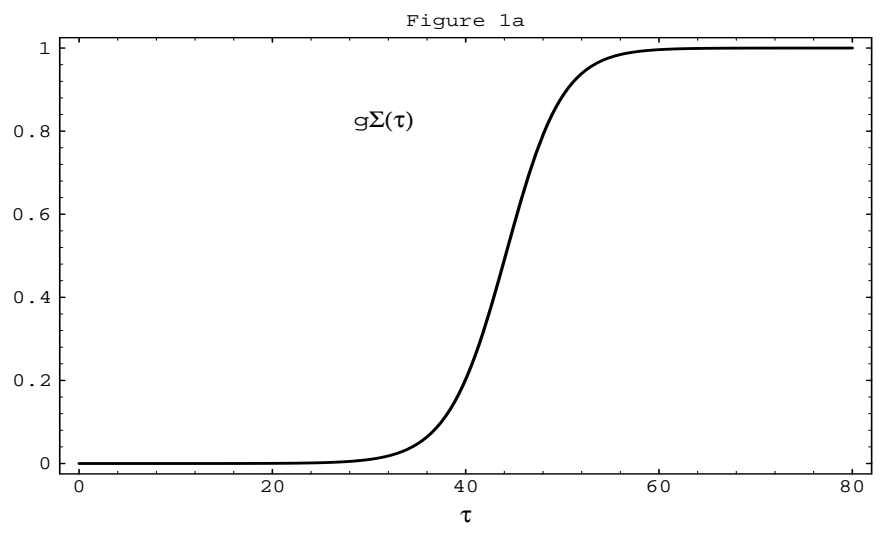

FIG. 10. $g \Sigma(\tau)$ vs. $\tau$ for $\eta(0)=\dot{\eta}(0)=0 ; \quad \lambda=10^{-12} ; \quad r=2 ; \quad h=2$.

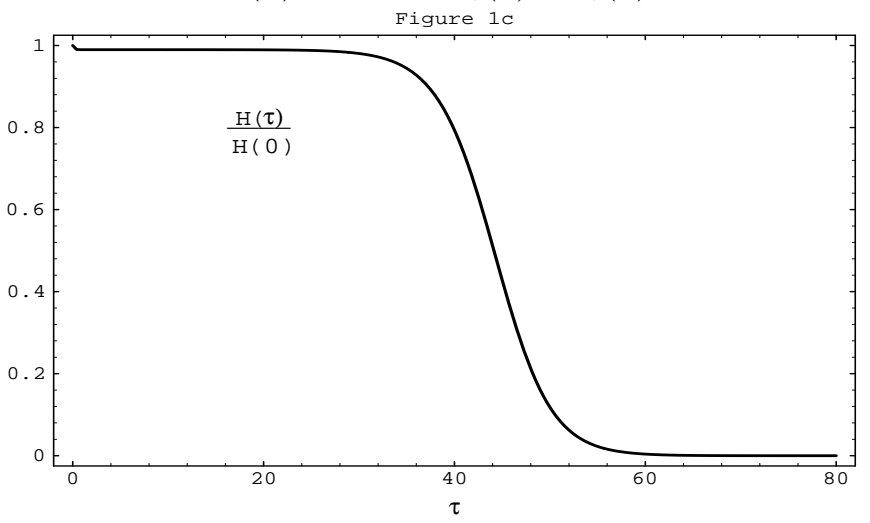

FIG. 11. $\mathcal{H}(\tau) / \mathcal{H}(0)$ vs $\tau$ for the same parameters as in fig.10.

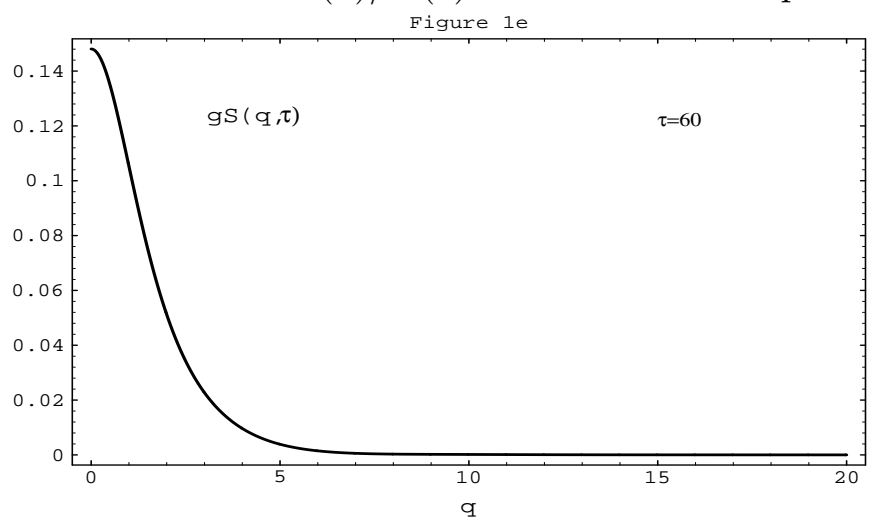

FIG. 12. $g \mathcal{S}(q, \tau)$ vs. $q$ for $\tau=60$. Same parameters as in fig.10.

These fluctuations translate into an amplification of the power spectrum at long wavelengths for $q \approx h$. To see this clearly we plot $g \mathcal{S}(q, \tau)=g\left|f_{q}(\tau)\right|^{2}$ vs. $q$ for $\tau=60$ in fig.12. This quantity is very small, because of the coupling constant in front, but for $\tau \approx \tau_{s}$ it grows to be of order one for long wavelengths (see also fig.14) and vanishes very fast for $q>10$. The integral in $g \Sigma(\tau)$ is dominated by these long wavelengths that become nonperturbatively large, whereas the contribution from the short wavelengths remains always perturbatively small. This is the justification for the approximations performed early that involved only the long-wavelength modes and cutoffs of order $\sqrt{h}$. The equal time spatial 
correlation function given by eq. (5.26) can now be computed explicitly. Figure 13 shows $S(\rho ; \tau) / S(0 ; \tau)$ as a function of $\rho$ for $\tau \geq 2$. We define the correlation length $\xi(\tau)$ as the value of $\rho$ for which the ratio is $1 / e$. Figure (1.g) shows $\xi(\tau)$; notice that the correlation length saturates to a value $\xi(\infty) \approx 1 / h$, and that the correlated regions are of horizon size.

We have performed numerical analysis varying $h$ with the same values of $g$ and for the same initial conditions, and found that the only quantitative change is in the time scale for $g \Sigma(\tau)$ to be of order one. We find that the spinodal time scale grows almost linearly with $h$ and its numerical value is accurately described by the estimate (8.10). The case in which the Hubble constant is $h=0.1$ is shown explicitly. Figure 15 shows $g \Sigma(\tau)$, which demonstrates the oscillatory behavior similar to what is seen in Minkowski space 10.11. The correlation length, $\xi(\tau)$, is shown in fig.16; its asymptotic value is again approximately given by $1 / h$.

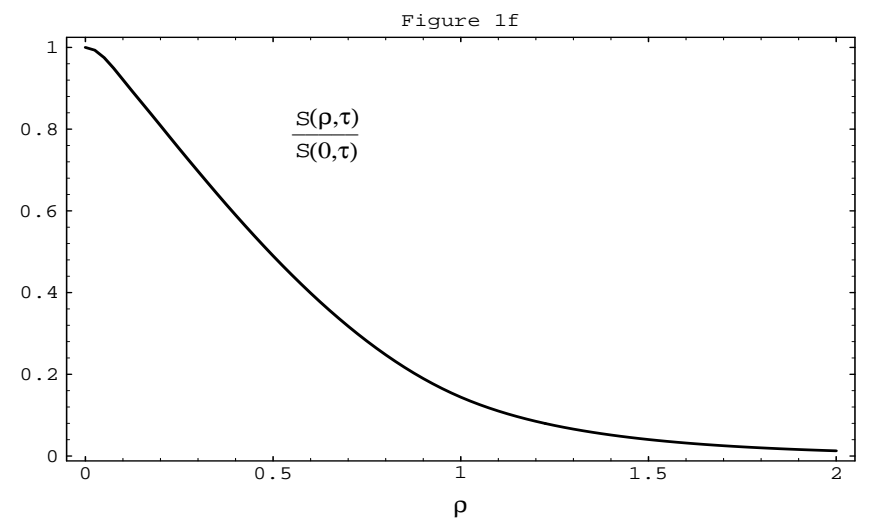

FIG. 13. $S(\rho, \tau) / S(0, \tau)$ vs. $\rho$ for $\tau \geq 2$. Same parameters as in fig.10.

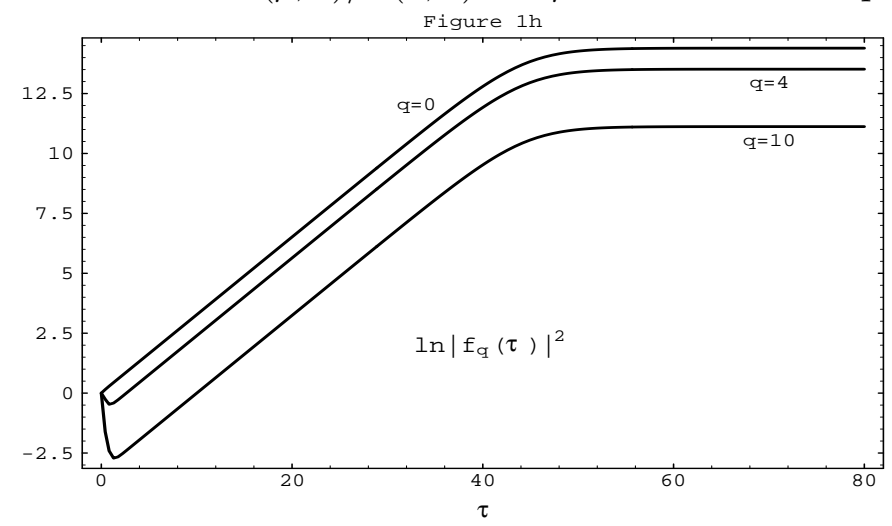

FIG. 14. $\ln \left[\left|f_{q}(\tau)\right|^{2}\right]$ vs. $\tau$ for $q=0,4,10$. Same parameters as in fig.10. 


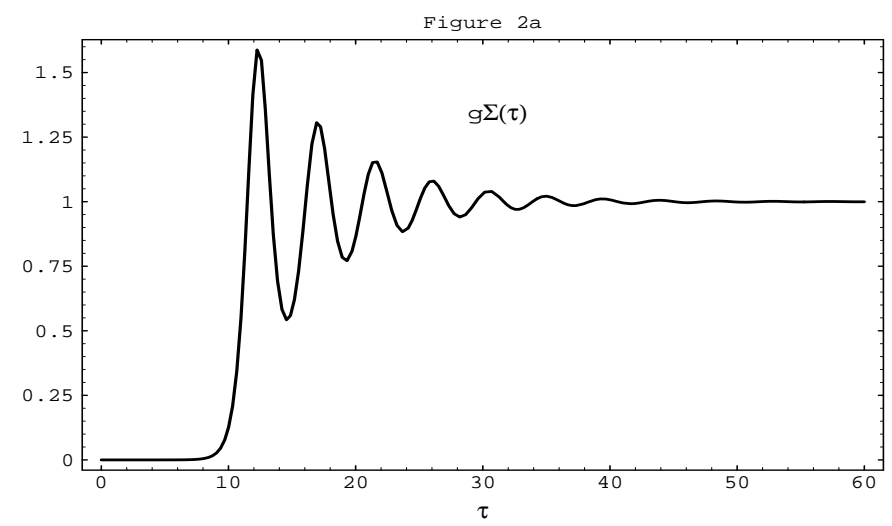

FIG. 15. $g \Sigma(\tau)$ vs. $\tau$ for $; \eta(0)=\dot{\eta}(0)=0 ; \quad ; \lambda=10^{-12} \quad r=2 ; \quad h=0.1$.

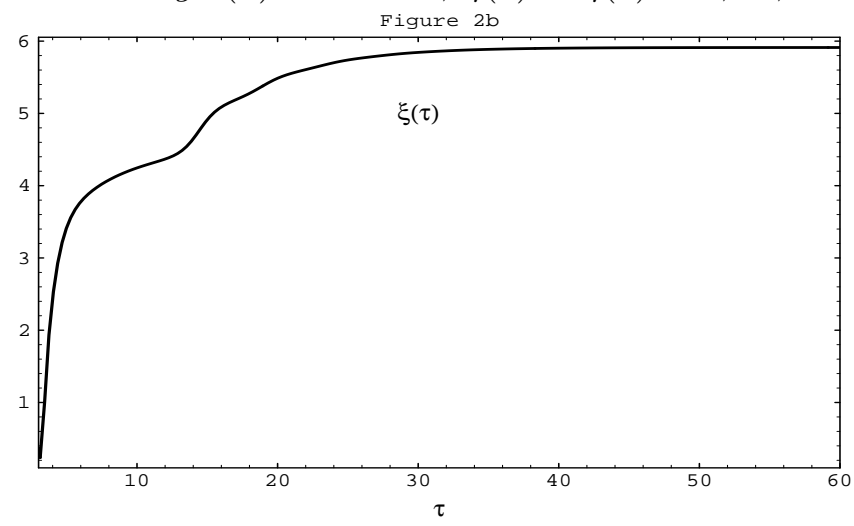

FIG. 16. $\xi(\tau)$ vs $\tau$. Same parameters as in fig. 15 .

\section{The late time limit}

For times $\tau>\tau_{s} \approx 40$ (for the values of the parameters used in figs. (1)) we see from figs. 10-14 that the dynamics freezes out. The fluctuation, $g \Sigma(\tau)=1$, and the mode functions effectively describe free, minimally coupled, massless particles. The sum rule,

$$
-1+g \Sigma(\infty)=0
$$

is obeyed exactly in the large $N$ limit as in the Minkowski case [10 12].

For the Hartree case $g \rightarrow 3 g$, but the physical phenomena are the same, with the only difference that the sum rule now becomes $g \Sigma(\infty)=1 / 3$. We now show that this value is a self-consistent solution of the equations of motion for the mode functions, and the only stationary solution for asymptotically long times.

In the late time limit, the effective time dependent mass term, $-1+\eta^{2}+g \Sigma$, in the equation for the mode functions, (5.18), vanishes (in this case with $\eta=0$ ). Therefore, these mode equations asymptotically become,

$$
\left[\frac{d^{2}}{d \tau^{2}}+3 h \frac{d}{d \tau}+\frac{q^{2}}{a^{2}(\tau)}\right] f_{q}(\tau)=0 .
$$

The general solutions are given by, 


$$
f_{q}^{a s y}(\tau)=\exp \left[-\frac{3}{2} h \tau\right]\left[c_{+}(q) J_{3 / 2}\left(\frac{q}{h} e^{-h \tau}\right)-i c_{-}(q) N_{3 / 2}\left(\frac{q}{h} e^{-h \tau}\right)\right],
$$

where $J_{3 / 2}(z)$ and $N_{3 / 2}(z)$ are the Bessel and Neumann functions, respectively. The coefficients, $c_{ \pm}(q)$ can be computed for large $q$ by matching $f_{q}^{a s y}(\tau)$ with the WKB approximation to the exact mode functions $f_{q}(\tau)$ that obey the initial conditions (5.19). The WKB approximation to $f_{q}(\tau)$ has been computed in ref. [13], and we find for large $q$,

$$
c_{ \pm}(q)=\sqrt{\frac{\pi q}{2 h}}\left[1-\frac{i}{q}(h+\Delta)+\mathcal{O}\left(q^{-2}\right)\right] e^{-i q / h} \pm \sqrt{\frac{\pi h}{8 q}}\left[1+\mathcal{O}\left(\frac{1}{q}\right)\right] e^{i q / h}
$$

where

$$
\Delta \equiv \int_{0}^{\infty} d \tau e^{h \tau} M^{2}(\tau)
$$

In the $\tau \rightarrow \infty$ limit, we have for fixed $q$,

$$
f_{q}^{a s y}(\tau) \stackrel{\tau \rightarrow \infty}{=} i \sqrt{\frac{2}{\pi}}\left(\frac{h}{q}\right)^{3 / 2} c_{-}(q)
$$

which are independent of time asymptotically, and explains why the power spectrum of quantum fluctuations freezes at times larger than the spinodal. This behavior is confirmed numerically: fig. 14 shows $\ln \left|f_{q}(\tau)\right|^{2}$ vs. $\tau$ for $q=0,4,10$. Clearly at early times the mode functions grow exponentially, and at times of the order of $\tau_{s}$, when $g \Sigma(\tau) \approx 1$ the mode functions freeze-out and become independent of time. Notice that the largest $q$ modes have grown the least, explaining why the integral is dominated by $q \leq 10-20$.

For asymptotically large times, $g \Sigma(\tau)$ is given by,

$$
g \Sigma(\infty)=g h^{2} \int_{0}^{+\infty} \frac{d q}{q} \operatorname{coth}\left(\frac{\omega_{q}}{2 T}\right)\left[\frac{2 h}{\pi}\left|c_{-}(q)\right|^{2}-q\right]
$$

where only one term in the UV subtraction survived in the $\tau=\infty$ limit. The factor $\operatorname{coth}\left(\frac{\omega_{q}}{2 T}\right)$ in eq. (8.22) takes into account the nonzero initial temperature $T$.

For consistency, this integral must converge and be equal to 1 as given by the sum rule. For this to be the case and to avoid the potential infrared divergence in (8.22), the coefficients $c_{-}(q)$ must vanish at $q=0$. The mode functions are finite in the $q \rightarrow 0$ limit provided,

$$
c_{-}(q) \stackrel{q \rightarrow 0}{=} \mathcal{C} q^{3 / 2}
$$

where $\mathcal{C}$ is a constant.

The numerical analysis and fig. 12 clearly show that the mode functions remain finite as $q \rightarrow 0$, and the coefficient $\mathcal{C}$ can be read off from these figures. This is a remarkable result. It is well known that for free massless minimally coupled fields in de Sitter space-time with Bunch-Davies boundary conditions, the fluctuation contribution $\left\langle\psi^{2}(\vec{x}, t)\right\rangle$ grows linearly in time as a consequence of the logarithmic divergence in the integrals [35]. However, in our case, although the asymptotic mode functions are free, the coefficients that multiply the Bessel functions of order $3 / 2$ have all the information of the interaction and initial 
conditions and must lead to the consistency of the sum rule. Clearly the sum rule and the initial conditions for the mode functions prevent the coefficients $c_{ \pm}(q)$ from describing the Bunch-Davies vacuum. These coefficients are completely determined by the initial conditions and the dynamics. This is the reason why the fluctuation freezes at long times unlike in the free case in which they grow linearly [35].

It is easy to see from eqs.(5.22)-(5.23) and (8.21) that the energy and pressure vanish for $\tau \rightarrow \infty$.

Analogously, the two point correlation function can be computed in the late time regime using the asymptotic results obtained above. Inserting eq.(8.18) for the mode functions in eq. (5.26) yields the asymptotic behavior:

$$
S(\rho, \tau) \stackrel{\tau \rightarrow \infty}{=} \frac{1}{4 \pi^{2} \rho} \int_{0}^{\infty} q d q \sin (q \rho) \operatorname{coth}\left(\frac{\omega_{q}}{2 T}\right) \frac{2 h^{3}}{\pi q^{3}}\left|c_{-}(q)\right|^{2} .
$$

The asymptotic behavior in time of the equal time correlation function is thus solely a function of $r$. The large $r$ behavior of $S(\vec{r},+\infty)$ is determined by the singularities of $\left|c_{-}(k)\right|^{2}$ in the complex $k$ plane. We find an exponential decrease,

$$
S(\rho,+\infty) \stackrel{\rho \rightarrow \infty}{\simeq} C \frac{e^{-\rho / \xi}}{\rho}
$$

where $\rho=i / \xi$ is the pole nearest to the real axis and $C$ is some constant. Thus we see that the freeze-out of the mode functions leads to the freeze-out of the correlation length $\xi$. The result of the numerical analysis is shown in fig. 16 which confirms this behavior and provides the asymptotic value for $\xi \approx 1 / h$. From these figures it is also clear that the freeze-out time is given by the expansion time scale, $1 / h$. More precisely, the numerical values for $\xi$ can be accurately reproduced by the following formula obtained by a numerical fit

$$
h \xi \simeq 1.02+0.2 \ln h+0.06 h+\ldots .
$$

This situation must be contrasted with that in Minkowski space-time [18] where the correlation length grows as $\xi(\tau) \approx \sqrt{\tau}$ during the stage of spinodal growth. Eventually, this correlation length saturates to a fairly large value that is typically several times larger than the zero temperature correlation length [18]. We see that in the de Sitter case the domains are always horizon-sized.

\section{Inflaton rolling down $(\eta(0) \neq 0)$ : classical or quantum behavior?}

Above we have analyzed the situation when $\eta(0)=0$ (or in dimensionful variables $\phi(0)=0)$. The typical analysis of inflaton dynamics in the literature involves the classical evolution of $\phi(t)$ with an initial condition in which $\phi(0)$ is very close to zero (i.e. the top of the potential hill) in the 'slow-roll' regime, for which $\ddot{\phi} \ll 3 H \dot{\phi}$. Thus, it is important to quantify the initial conditions on $\phi(t)$ for which the dynamics will be determined by the classical evolution of $\phi(t)$ and those for which the quantum fluctuations dominate the dynamics. We can provide a criterion to separate classical from quantum dynamics by analyzing the relevant time scales, estimated by neglecting non-linearities and backreaction 
effects. We consider the evolution of the zero mode in terms of dimensionless variables, and choose $\eta(0) \neq 0$ and $\dot{\eta}(0)=0$. $(\dot{\eta}(0) \neq 0$ simply corresponds to a shift in origin of time $)$. We assume $\eta(0)^{2}<<1$ which is the relevant case where spinodal instabilities are important. We find

$$
\eta(\tau) \approx \eta(0) e^{\left(\nu-\frac{3}{2}\right) h_{0} \tau}
$$

The non-linearities will become important and eventually terminate inflation when $\eta(\tau) \approx 1$. This corresponds to a time scale given by

$$
\tau_{c} \approx \frac{\ln [1 / \eta(0)]}{\left(\nu-\frac{3}{2}\right) h_{0}} .
$$

Comparing this time scale to the spinodal time scale given by (8.10), for which quantum fluctuations grow to be of $\mathcal{O}(1)$, we see that when,

$$
\eta(0)<<g^{1 / 4}
$$

the quantum fluctuations will grow to be $\mathcal{O}(1)$ much earlier than the zero mode for $T_{i}>T_{c}$ (for $T_{i}=0$ the bound becomes $\eta(0)<<g^{1 / 2}$ ). In this case the dynamics will be driven completely by the quantum fluctuations, as the zero mode will be rolling down the potential hill very slowly and will not grow enough to compete with the quantum fluctuations before the fluctuations grow to overcome the tree level terms in the equations of motion. In this case, as argued previously, the large $N$ and Hartree approximations will be completely equivalent during the time scales of interest.

On the other hand, if

$$
\eta(0)>>g^{1 / 4}
$$

then the zero mode will roll and become $\mathcal{O}(1)$ before the fluctuations have enough time to grow to $\mathcal{O}(1)\left(\eta(0)>>g^{1 / 2}\right.$ for $\left.T_{i}=0\right)$. In this case, the dynamics will be dominated by the rolling of the zero mode and is mostly classical. The quantum fluctuations remain perturbatively small throughout the inflationary stage which will end when the velocity of the zero mode modifies the equation of state to terminate de Sitter expansion.

For $\eta(0) \approx g^{1 / 4}$ (or $\eta(0) \approx g^{1 / 2}$ for $\left.T_{i}=0\right)$, both the rolling of the zero mode and the quantum fluctuations will give contributions of the same order to the dynamics. In this case, the quantum fluctuations will be large for the long-wavelength modes and the classical approximation to the inflationary dynamics will not be accurate.

Since the scenario in which $\eta(0)>>g^{1 / 4}$, in which the dynamics is basically driven by the classical evolution of the zero mode has received a great deal of attention in the literature, we will not focus on this case, but instead analyze numerically the cases in which $\eta(0) \neq 0$ but such that $\eta(0) \leq g^{1 / 4}$.

\section{E. Numerical Analysis:}

We have evolved the set of equations of motion given by (5.18) numerically with initial conditions (5.19) for the large $N$ case, and (4.27) and (4.28), with the corresponding initial 
conditions (4.30) on the mode functions for the Hartree case. The numerical code is the same as in the previous section with the same relative errors.

\section{Large N case:}

Figs. $17-18$ show $\eta(\tau)$ and $g \Sigma(\tau)$ vs. $\tau$ for the values $g=10^{-12} ; \quad T_{i} / T_{c}=2 ; \quad \eta(0)=$ $10^{-5} ; \quad \dot{\eta}(0)=0$. Clearly the dynamics is dominated by the fluctuations; the zero mode grows but is always negligible small compared to $g \Sigma(\tau)$. The time scale at which $g \Sigma(\tau)$ grows to be of order one is about the same as in the case, $\eta(0)=0$, and all the behavior for the mode functions, correlation length, energy density, pressure, etc. is similar to the case analyzed in the previous section.

Asymptotically, we find that the sum rule (5.20) is satisfied to our numerical accuracy. This is the same as the situation in Minkowski space-time [10,11], and when $\eta \neq 0$, this sum rule is nothing but the Ward identity associated with Goldstone's theorem. The fluctuations are Goldstone bosons, minimally coupled, and the symmetry is spontaneously broken with a very small expectation value for the order parameter as can be read off from fig.17. For $\tau>\tau_{s}$, the dynamics freezes completely and the zero mode and the fluctuations achieve their asymptotic values much in the same way as in the case $\eta=0$ studied in the previous section. Again, the correlation length becomes independent of time with $\xi(\infty) \approx 1 / h$ in a time scale given by $1 / h$.

Because there is a damping term in the zero mode equation, it is reasonable to assume that asymptotically there will be a solution with a constant value of $\eta$. Then the Wardidentity, $\eta(\infty)\left[-1+\eta^{2}(\infty)+g \Sigma(\infty)\right]=0$, must be fulfilled. In the large $N$ case, the only stationary solutions are i) $\eta=0 ; g \Sigma(\infty)=1$, or, ii) $\eta(\infty) \neq 0 ;-1+\eta^{2}(\infty)+g \Sigma(\infty)=0$. To have a consistent solution of the mode functions, it must be that the effective mass term $\left[-1+\eta^{2}(\infty)+g \Sigma(\infty)\right]$ vanishes asymptotically, leading to the mode equations for massless, minimally coupled modes which are asymptotically independent of time as shown in the previous section (see eq.(8.21)). Furthermore, from fig.18 it is clear that $g \Sigma(\tau)$ remains constant at long times, again unlike the case of free massless fields with Bunch-Davies boundary conditions in which case the fluctuation grows linearly in time [35].

\section{Hartree case:}

Figure 17 also shows the evolution of the zero mode in the large $N$ and Hartree case. Although there is a quantitative difference in the amplitude of the zero mode, in both cases it is extremely small and gives a negligible contribution to the dynamics. In the Hartree case, however, there is no equivalent of the large $N$ sum rule; the only stationary solution for $\eta \neq 0$ is, $\eta^{2}(\infty)=1 ; g \Sigma(\infty)=0$. Such a solution leads to mode equations with a positive mass term and mode functions that vanish exponentially fast for $\tau \rightarrow \infty$ for all momenta. However, whether the asymptotic behavior of the Hartree solution is achieved within the interesting time scales is a matter of initial conditions. For example in fig.17 the initial condition is such that the time scale for growth of the quantum fluctuations is much shorter than the time scale for which the amplitude of the zero mode grows large and the non-linearities become important. In the large $N$ case the sum rule is satisfied with a large value of the quantum fluctuations. In the Hartree case the equivalent sum rule $-1+3 \eta_{H}^{2}+3 g \Sigma_{H}=0$ is satisfied for a very small $\eta_{H}$ and a $g \Sigma_{H} \approx 1 / 3$. The modes become effectively massless and they stop growing. 


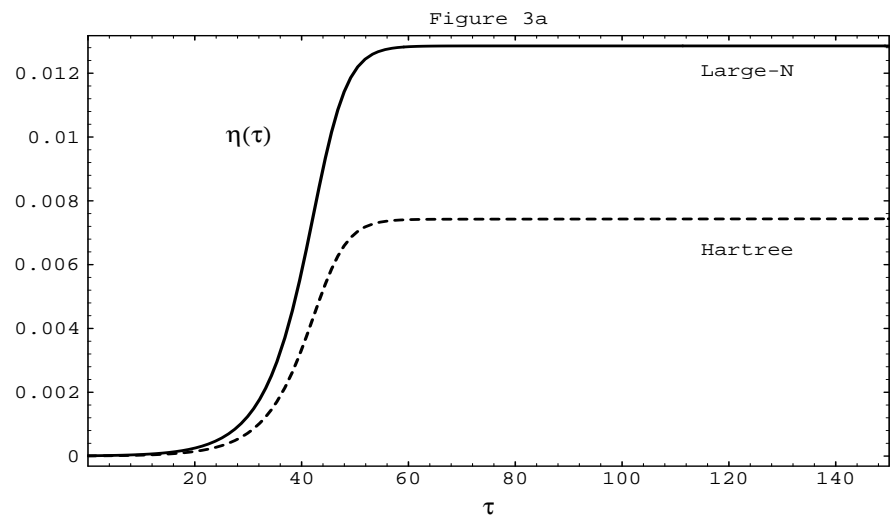

FIG. 17. $\quad \eta(\tau)$ vs. $\tau$ for $\lambda=10^{-12} ; \quad r=2 ; \quad h=2 ; \quad \eta(0)=10^{-5} ; \quad \dot{\eta}(0)=0$ for large $N$ (solid curve) and Hartree (dashed curve).

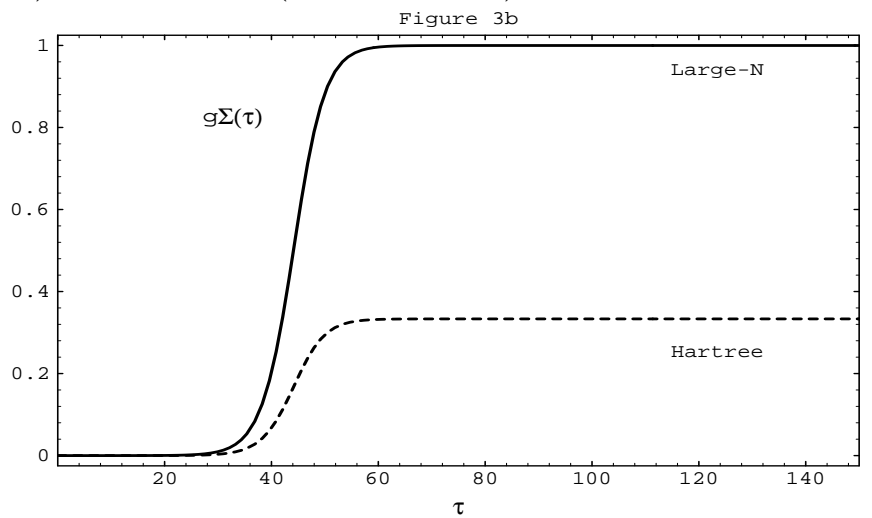

FIG. 18. $g \Sigma(\tau)$ vs. $\tau$ for the same values of the parameters as in fig.17, for large $N$ (solid curve) and Hartree (dashed curve).

The equation for the zero mode (see eq.(4.27)) still has an uncancelled piece of the nonlinearity, $-2 \eta^{2}$; however the derivatives and the amplitude of $\eta$ are all extremely small and though the zero mode still evolves in time, it does so extremely slowly. In fact the Hartree curve in fig. 17 has an extremely small positive slope asymptotically, and while $\eta_{H}$ grows very slowly, $g \Sigma_{H}$ diminishes at the same rate. In the case shown in fig.17, we find numerically that $\dot{\eta}_{H} / \eta_{H} \approx 10^{-7}$ at $\tau=150$. Before this time most of the interesting dynamics that can be captured with a fixed de Sitter background had already taken place, and the backreaction of the fluctuations on the metric becomes substantial requiring an analysis that treats the scale factor dynamically.

The conclusion of our analysis is that in the region of initial conditions for which the quantum fluctuations dominate the dynamics, that is for $\eta(0)<<g^{1 / 4}$, both large $N$ and Hartree give the same answer on the relevant time scales. The figures for $\mathcal{H}(\tau) / \mathcal{H}(0)$ are numerically indistinguishable from the case of figs. 1.

We see that in the large $N$ case the zero mode rolls to a final amplitude which is $\mathcal{O}(1)$ and of the same order as $g \Sigma(\infty)$ and the sum rule is satisfied. However, the Hartree case clearly shows the asymptotics analyzed above with $\eta_{H}(\infty)=1 ; g \Sigma_{H}(\infty)=0$.

This particular borderline case is certainly not generic and would imply some fine tuning of initial conditions. Finally the case in which $\eta(0)>>g^{1 / 4}$ (or $g^{1 / 2}$ for $T_{i}=0$ ) is basically classical in that the dynamics is completely given by the classical rolling of the zero mode 
and the fluctuations are always perturbatively small.

\section{F. Discussion and Conclusions for the de Sitter background}

We have identified analytically and numerically two distinct regimes for the dynamics determined by the initial condition on the expectation value of the zero mode of the inflaton 15.

1. When $\eta(0)<<g^{1 / 4}$ (or $g^{1 / 2}$ for $T_{i}=0$ ), the dynamics is driven by quantum (and thermal) fluctuations. Spinodal instabilities grow and eventually compete with tree level terms at a time scale, $\tau_{s} \geq-3 h \ln [g] / 2$. The growth of spinodal fluctuations translates into the growth of spatially correlated domains which attain a maximum correlation length (domain size) of the order of the horizon. For very weak coupling and $h \geq 1$ this time scale can easily accommodate enough e-folds for inflation to solve the flatness and horizon problems. The quantum fluctuations modify the equation of state dramatically providing a means for a graceful exit to the inflationary stage without slow-roll.

This non-perturbative description of the non-equilibrium effects in this regime in which quantum (and thermal) fluctuations are most important is borne out by both the large $N$ and Hartree approximations. Thus our analysis provides a reliable understanding of the relevant non-perturbative, non-equilibrium effects of the fluctuations that have not been revealed before in this setting [10] - [16].

These initial conditions are rather natural if the de Sitter era arises during a phase transition from a radiation dominated high temperature phase in local thermodynamic equilibrium, in which the order parameter and its time derivative vanish.

2. When $\eta(0)>>g^{1 / 4}$ (or $g^{1 / 2}$ for $T_{i}=0$ ), the dynamics is driven solely by the classical evolution of the inflaton zero mode. The quantum and thermal fluctuations are always perturbatively small (after renormalization), and their contribution to the dynamics is negligible for weak couplings. The de Sitter era will end when the kinetic contribution to the energy becomes of the same order as the 'vacuum' term. This is the realm of the slow-roll analysis whose characteristics and consequences have been analyzed in the literature at length. These initial conditions, however, necessarily imply some initial state either with a biasing field that favors a non-zero initial expectation value, or that in the radiation dominated stage, prior to the phase transition, the state was strongly out of equilibrium with an expectation value of the zero mode different from zero. Although such a state cannot be ruled out and would naturally arise in chaotic scenarios, the description of the phase transition in this case requires further input on the nature of the state prior to the phase transition.

\section{SELF-CONSISTENT EVOLUTION OF MATTER FIELDS WITH A DYNAMICAL COSMOLOGICAL BACKGROUND}

We present in this section the full self-consistent matter-geometry dynamics [16]. That is, the scale factor $a(t)$ is here a dynamical variable determined by the Einstein-Friedman 
eq. (5.21)-(5.22) coupled with the matter evolution eqs.(5.18)-(5.19).

In order to provide the full solution we now must provide the values of $\eta(0), \dot{\eta}(0)$, and $h_{0}$. Assuming that the inflationary epoch is associated with a phase transition at the GUT scale, this requires that $N m_{R}^{4} / g \approx\left(10^{15} \mathrm{Gev}\right)^{4}$ and assuming the bound on the scalar selfcoupling $g \approx 10^{-12}-10^{-14}$ (this will be seen later to be a compatible requirement), we find that $h_{0} \approx N^{1 / 4}$ which we will take to be reasonably given by $h_{0} \approx 1-10$ (for example in popular GUT's $N \approx 20$ depending on particular representations).

We will begin by studying the case of most interest from the point of view of describing the phase transition: $\eta(0)=0$ and $\dot{\eta}(0)=0$, which are the initial conditions that led to puzzling questions. With these initial conditions, the evolution equation for the zero mode eq.(5.18) determines that $\eta(\tau)=0$ by symmetry.

\section{A. Early time dynamics:}

Before engaging in the numerical study, it proves illuminating to obtain an estimate of the relevant time scales and an intuitive idea of the main features of the dynamics. Because the coupling is so weak $\left(g \sim 10^{-12} \ll 1\right)$ and after renormalization the contribution from the quantum fluctuations to the equations of motion is finite, we can neglect all the terms proportional to $g$ in eqs. (5.22) and (5.18).

For the case where we choose $\eta(\tau)=0$ and the evolution equations for the mode functions are those for an inverted oscillator in De Sitter space-time, which have been studied in sec. VIII 38]. One obtains the approximate solutions (8.3)-(8.4).

After the physical wavevectors cross the horizon, i.e. when $q e^{-h_{0} \tau} / h_{0} \ll 1$ we find that the mode functions factorize:

$$
f_{q}(\tau) \approx \frac{B_{q}}{\Gamma(1-\nu)}\left(\frac{2 h_{0}}{q}\right)^{\nu} e^{(\nu-3 / 2) h_{0} \tau}
$$

This result reveals a very important feature: because of the negative mass squared term in the matter Lagrangian leading to symmetry breaking (and $\nu>3 / 2$ ), we see that all of the mode functions grow exponentially after horizon crossing (for positive mass squared $\nu<3 / 2$, and they would decrease exponentially after horizon crossing). This exponential growth is a consequence of the spinodal instabilities which is a hallmark of the process of phase separation that occurs to complete the phase transition. We note, in addition that the time dependence is exactly given by that of the $q=0$ mode, i.e. the zero mode, which is a consequence of the redshifting of the wavevectors and the fact that after horizon crossing the contribution of the term $q^{2} / a^{2}(\tau)$ in the equations of motion become negligible. We clearly see that the quantum fluctuations grow exponentially and they will begin to be of the order of the tree level terms in the equations of motion when $g \Sigma(\tau) \approx 1$. At large times

$$
\Sigma(\tau) \approx \mathcal{F}^{2}\left(h_{0}\right) h_{0}^{2} e^{(2 \nu-3) h_{0} \tau}
$$

with $\mathcal{F}\left(h_{0}\right)$ a finite constant that depends on the initial conditions and is found numerically to be of $\mathcal{O}(1)$ [see fig.24]. 
In terms of the initial dimensionful variables, the condition $g \Sigma(\tau) \approx 1$ translates to $<\psi^{2}(\vec{x}, t)>_{R} \approx 2 m_{R}^{2} / g$, i.e. the quantum fluctuations sample the minima of the (renormalized) tree level potential. We find that the time at which the contribution of the quantum fluctuations becomes of the same order as the tree level terms is estimated to be [15]

$$
\tau_{s} \approx \frac{1}{(2 \nu-3) h_{0}} \ln \left[\frac{1}{g h_{0}^{2} \mathcal{F}^{2}\left(h_{0}\right)}\right]=\frac{3}{2} h_{0} \ln \left[\frac{(2 \pi)^{2}}{g h_{0}^{2} \mathcal{F}^{2}\left(h_{0}\right)}\right]+\mathcal{O}\left(1 / h_{0}\right) .
$$

At this time, the contribution of the quantum fluctuations makes the back reaction very important and, as will be seen numerically, this translates into the fact that $\tau_{s}$ also determines the end of the De Sitter era and the end of inflation. The total number of e-folds during the stage of exponential expansion of the scale factor (constant $\left.h_{0}\right)$ is given by

$$
N_{e} \approx \frac{1}{2 \nu-3} \ln \left[\frac{1}{g h_{0}^{2} \mathcal{F}^{2}\left(h_{0}\right)}\right]=\frac{3}{2} h_{0}^{2} \ln \left[\frac{(2 \pi)^{2}}{q h_{0}^{2} \mathcal{F}^{2}\left(h_{0}\right)}\right]+\mathcal{O}(1)
$$

For large $h_{0}$ we see that the number of e-folds scales as $h_{0}^{2}$ as well as with the logarithm of the inverse coupling. These results (9.1-9.3) will be confirmed numerically below and will be of paramount importance for the interpretation of the main consequences of the dynamical evolution.

As discussed in sec. VIII.D, the early time dynamics is dominated by classical or quantum effects depending on the ratio between the time scales $\tau_{c}$ and $\tau_{s}$.

If $\tau_{c}$ is much smaller than the spinodal time $\tau_{s}$ given by eq.(9.2) then the classical evolution of the zero mode will dominate the dynamics and the quantum fluctuations will not become very large, although they will still undergo spinodal growth. On the other hand, if $\tau_{c} \gg \tau_{s}$ the quantum fluctuations will grow to be very large well before the zero mode reaches the non-linear regime. In this case the dynamics will be determined completely by the quantum fluctuations. Then the criterion for the classical or quantum dynamics is given by

$$
\begin{aligned}
& \eta(0) \gg \sqrt{g} h_{0} \Longrightarrow \text { classical dynamics } \\
& \eta(0) \ll \sqrt{g} h_{0} \Longrightarrow \text { quantum dynamics }
\end{aligned}
$$

or in terms of dimensionful variables $\phi(0) \gg H_{0}$ leads to classical dynamics and $\phi(0) \ll H_{0}$ leads to quantum dynamics.

However, even when the classical evolution of the zero mode dominates the dynamics, the quantum fluctuations grow exponentially after horizon crossing unless the value of $\phi(t)$ is very close to the minimum of the tree level potential. In the large $N$ approximation the spinodal line, that is the values of $\phi(t)$ for which there are spinodal instabilities, reaches all the way to the minimum of the tree level potential as can be seen from the equations of motion for the mode functions. Therefore even in the classical case one must understand how to deal with quantum fluctuations that grow after horizon crossing.

\section{B. Numerics}

The time evolution is carried out by means of a fourth order Runge-Kutta routine with adaptive step-sizing while the momentum integrals are carried out using an 11-point NewtonCotes integrator. The relative errors in both the differential equation and the integration 
are of order $10^{-8}$. We find that the energy is covariantly conserved throughout the evolution to better than a part in a thousand. Figs. 1921 show $g \Sigma(\tau)$ vs. $\tau, h(\tau)$ vs. $\tau$ and $\ln \left|f_{q}(\tau)\right|^{2}$ vs. $\tau$ for several values of $q$ with larger $q^{\prime} s$ corresponding to successively lower curves. Figs. 22,23 show $p(\tau) / \varepsilon(\tau)$ and the horizon size $h^{-1}(\tau)$ for $g=10^{-14} ; \eta(0)=0 ; \dot{\eta}(0)=0$ and we have chosen the representative value $h_{0}=2.0$.
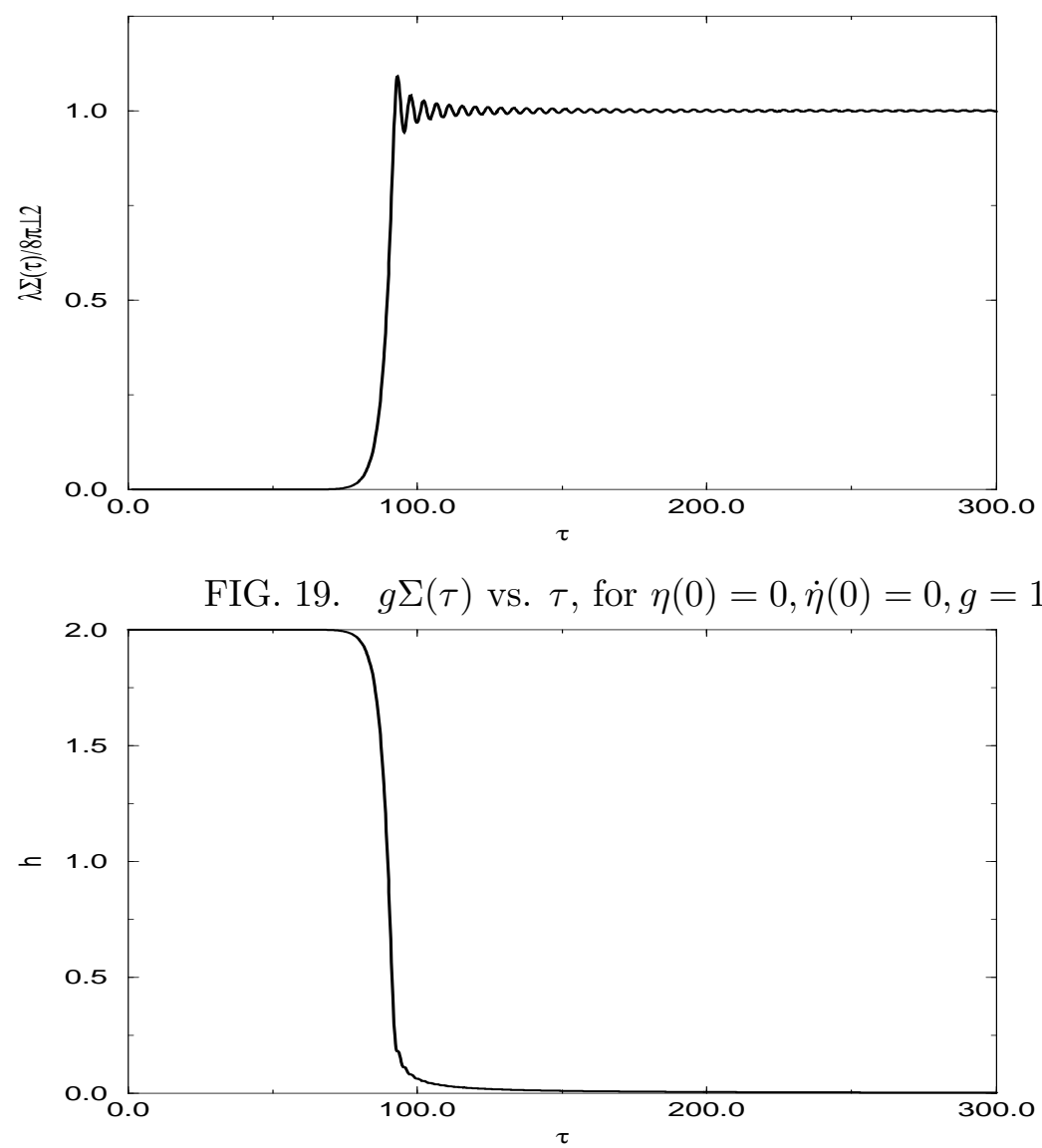

FIG. 20. $H(\tau)$ vs. $\tau$, for $\eta(0)=0, \dot{\eta}(0)=0, g=10^{-14}, h_{0}=2.0$.

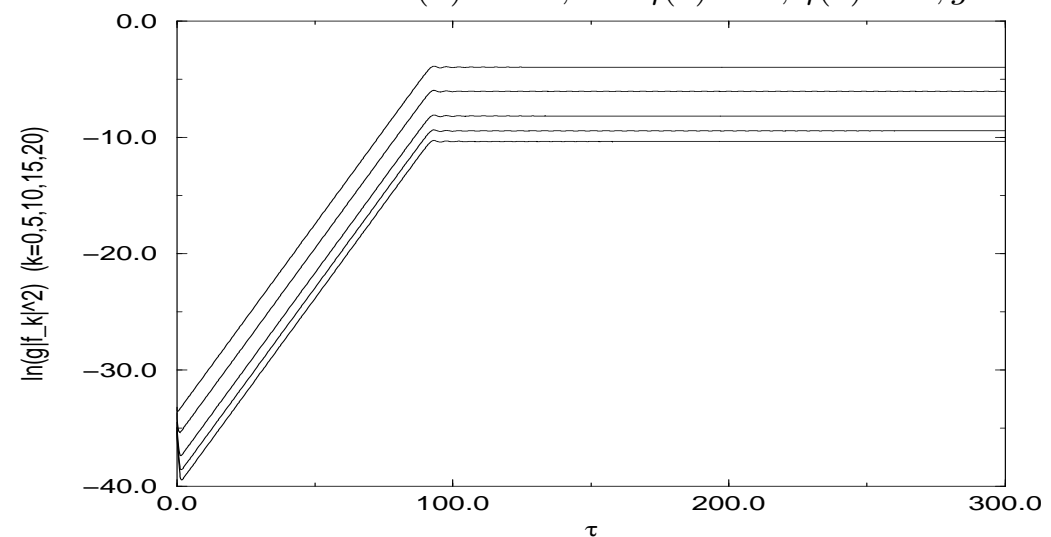

FIG. 21. $\ln \left|f_{q}(\tau)\right|^{2}$ vs. $\tau$, for $\eta(0)=0, \dot{\eta}(0)=0, g=10^{-14}, h_{0}=2.0$ for $q=0.0,5,10,15,20$ with smaller $q$ corresponding to larger values of $\ln \left|f_{q}(\tau)\right|^{2}$.

Figs. 19 and 20 show clearly that when the contribution of the quantum fluctuations $g \Sigma(\tau)$ becomes of order 1 inflation ends, and the time scale for $g \Sigma(\tau)$ to reach $\mathcal{O}(1)$ is very 
well described by the estimate (9.2). From fig.19 we see that this happens for $\tau=\tau_{s} \approx 90$, leading to a number of e-folds $N_{e} \approx 180$ which is correctly estimated by (9.2, 9.3).

Fig. 21 shows clearly the factorization of the modes after they cross the horizon as described by eq.(9.1). The slopes of all the curves after they become straight lines in fig.21 is given exactly by $(2 \nu-3)$, whereas the intercept depends on the initial condition on the mode function and the larger the value of $q$ the smaller the intercept because the amplitude of the mode function is smaller initially. Although the intercept depends on the initial conditions on the long-wavelength modes, the slope is independent of the value of $q$ and is the same as what would be obtained in the linear approximation for the square of the zero mode at times long enough that the decaying solution can be neglected but short enough that the effect of the non-linearities is very small. Notice from the figure that when inflation ends and the non-linearities become important all of the modes effectively saturate. This is also what one would expect from the solution of the zero mode: exponential growth in early-intermediate times (neglecting the decaying solution), with a growth exponent given by $(\nu-3 / 2)$ and an asymptotic behavior of small oscillations around the equilibrium position, which for the zero mode is $\eta=1$, but for the $q \neq 0$ modes depends on the initial conditions. All of the mode functions have this behavior once they cross the horizon. We have also studied the phases of the mode functions and we found that they freeze after horizon crossing in the sense that they become independent of time. This is natural since both the real and imaginary parts of $f_{q}(\tau)$ obey the same equation but with different boundary conditions. After the physical wavelength crosses the horizon, the dynamics is insensitive to the value of $q$ for real and imaginary parts and the phases become independent of time. Again, this is a consequence of the factorization of the modes.

The growth of the quantum fluctuations is sufficient to end inflation at a time given by $\tau_{s}$ in eq.(9.2). Furthermore fig. 22 shows that during the inflationary epoch $p(\tau) / \varepsilon(\tau) \approx-1$ and the end of inflation is rather sharp at $\tau_{s}$ with $p(\tau) / \varepsilon(\tau)$ oscillating between \pm 1 with zero average over the cycles, resulting in matter domination. Fig. 23] shows this feature very clearly; $h(\tau)$ is constant during the de Sitter epoch and becomes matter dominated after the end of inflation with $h^{-1}(\tau) \approx \frac{3}{2}\left(\tau-\tau_{s}\right)$. There are small oscillations around this value because both $p(\tau)$ and $\varepsilon(\tau)$ oscillate. These oscillations are a result of small oscillations of the mode functions after they saturate, and are also a feature of the solution for a zero mode.

All of these features hold for a variety of initial conditions. As an example, we show in ref. [16] the case of an initial Hubble parameter of $h_{0}=10$. 


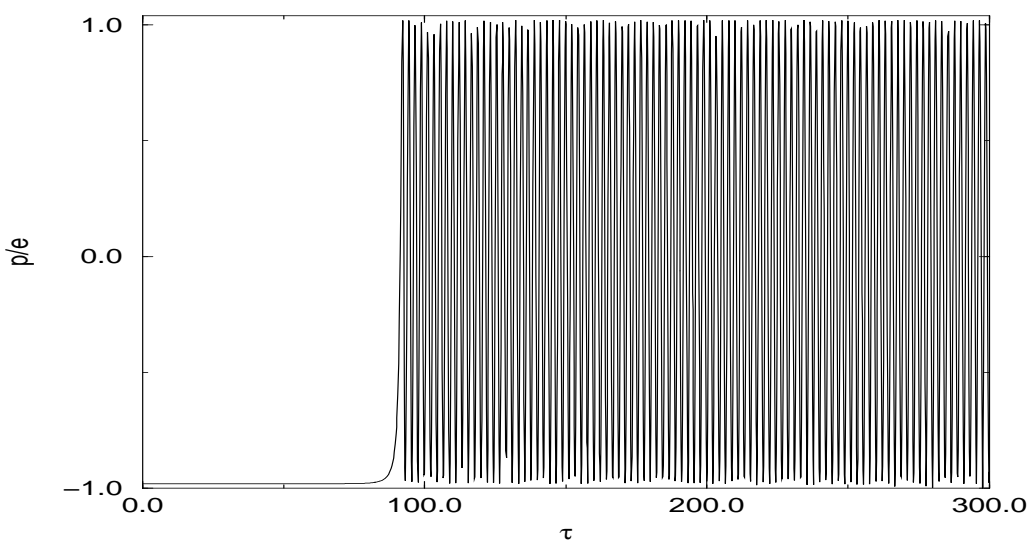

FIG. 22. $p / \varepsilon$ vs. $\tau$, for $\eta(0)=0, \dot{\eta}(0)=0, g=10^{-14}, h_{0}=2.0$.

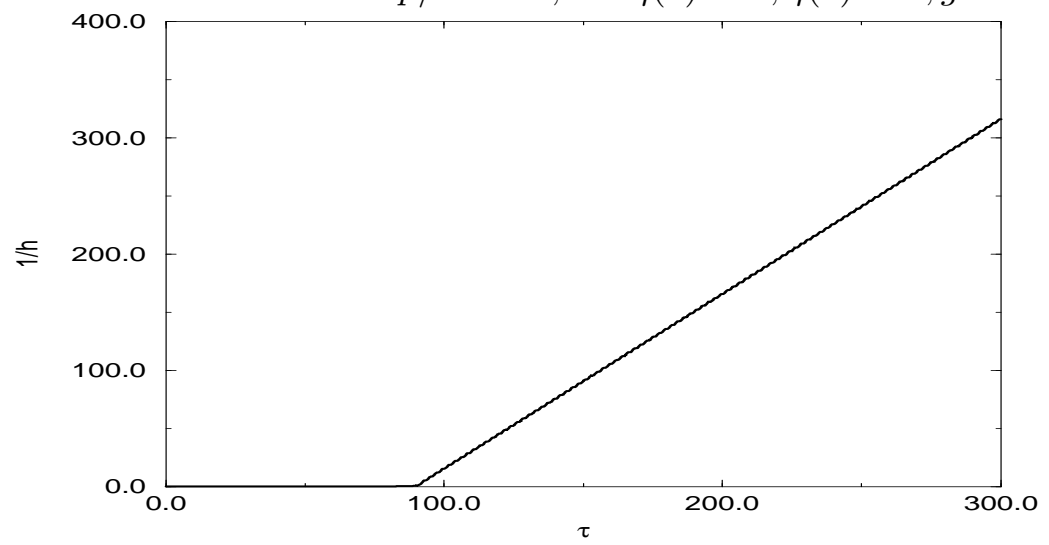

FIG. 23. $1 / h(\tau)$ vs. $\tau$, for $\eta(0)=0, \dot{\eta}(0)=0, g=10^{-14}, h_{0}=2.0$.

\section{Zero Mode Assembly:}

This remarkable feature of factorization of the mode functions after horizon crossing can be elegantly summarized as

$$
\left.f_{k}(t)\right|_{k_{p h}(t) \ll H}=g(q, h) f_{0}(\tau),
$$

with $k_{p h}(t)=k e^{-H t}$ being the physical momentum, $g(q, h)$ a complex constant, and $f_{0}(\tau)$ a real function of time that satisfies the mode equation with $q=0$ and real initial conditions which will be inferred later. Since the factor $g(q, h)$ depends solely on the initial conditions on the mode functions, it turns out that for two mode functions corresponding to momenta $k_{1}, k_{2}$ that have crossed the horizon at times $t_{1}>t_{2}$, the ratio of the two mode functions at time $t,\left(t_{s}>t>t_{1}>t_{2}\right)$ is

$$
\frac{f_{k_{1}}(t)}{f_{k_{2}}(t)} \propto e^{\left(\nu-\frac{3}{2}\right) h\left(\tau_{1}-\tau_{2}\right)}>1
$$

Then if we consider the contribution of these modes to the renormalized quantum fluctuations a long time after the beginning of inflation (so as to neglect the decaying solutions), we find that

$$
g \Sigma(\tau) \approx \mathcal{C} e^{(2 \nu-3) h \tau}+\text { small }
$$


where 'small' stands for the contribution of mode functions associated with momenta that have not yet crossed the horizon at time $\tau$, which give a perturbatively small (of order $g$ ) contribution. We find that several e-folds after the beginning of inflation but well before inflation ends, this factorization of superhorizon modes implies the following:

$$
\begin{aligned}
g \int q^{2} d q\left|f_{q}^{2}(\tau)\right| & \approx\left|C_{0}\right|^{2} f_{0}^{2}(\tau), \\
g \int q^{2} d q\left|\dot{f}_{q}^{2}(\tau)\right| & \approx\left|C_{0}\right|^{2} \dot{f}_{0}^{2}(\tau), \\
g \int \frac{q^{4}}{a^{2}(\tau)} d q\left|f_{q}^{2}(\tau)\right| & \approx \frac{\left|C_{1}\right|^{2}}{a^{2}(\tau)} f_{0}^{2}(\tau),
\end{aligned}
$$

where we have neglected the weak time dependence arising from the perturbatively small contributions of the short-wavelength modes that have not yet crossed the horizon, and the integrals above are to be understood as the fully renormalized (subtracted), finite integrals. For $\eta=0$, we note that (9.6) and the fact that $f_{0}(\tau)$ obeys the equation of motion for the mode with $q=0$ leads at once to the conclusion that in this regime $[g \Sigma(\tau)]^{\frac{1}{2}}=\left|C_{0}\right| f_{0}(\tau)$ obeys the zero mode equation of motion

$$
\left[\frac{d^{2}}{d \tau^{2}}+3 h \frac{d}{d \tau}-1+\left(\left|C_{0}\right| f_{0}(\tau)\right)^{2}\right]\left|C_{0}\right| f_{0}(\tau)=0 .
$$

It is clear that several e-folds after the beginning of inflation, we can define an effective zero mode as

$$
\eta_{e f f}^{2}(\tau) \equiv g \Sigma(\tau), \text { or in dimensionful variables, } \phi_{e f f}(t) \equiv\left[\left\langle\psi^{2}(\vec{x}, t)\right\rangle_{R}\right]^{\frac{1}{2}}
$$

Although this identification seems natural, we emphasize that it is by no means a trivial or ad-hoc statement. There are several important features that allow an unambiguous identification: i) $\left[\left\langle\psi^{2}(\vec{x}, t)\right\rangle_{R}\right]$ is a fully renormalized operator product and hence finite, ii) because of the factorization of the superhorizon modes that enter in the evaluation of $\left[\left\langle\psi^{2}(\vec{x}, t)\right\rangle_{R}\right]$, $\phi_{\text {eff }}(t)$ (9.10) obeys the equation of motion for the zero mode, iii) this identification is valid several e-folds after the beginning of inflation, after the transient decaying solutions have died away and the integral in $\left\langle\psi^{2}(\vec{x}, t)\right\rangle$ is dominated by the modes with wavevector $k$ that have crossed the horizon at $t(k) \ll t$. Numerically we see that this identification holds throughout the dynamics except for a very few e-folds at the beginning of inflation. This factorization determines at once the initial conditions of the effective zero mode that can be extracted numerically: after the first few e-folds and long before the end of inflation we find

$$
\phi_{e f f}(t) \equiv \phi_{e f f}(0) e^{\left(\nu-\frac{3}{2}\right) H t}
$$

where we parameterized

$$
\phi_{\text {eff }}(0) \equiv \frac{H}{2 \pi} \mathcal{F}(H / m)
$$

to make contact with the literature. As is shown in fig. (24), we find numerically that $\mathcal{F}(H / m) \approx \mathcal{O}(1)$ for a large range of $0.1 \leq H / m \leq 50$ and that this quantity depends on the initial conditions of the long wavelength modes. 


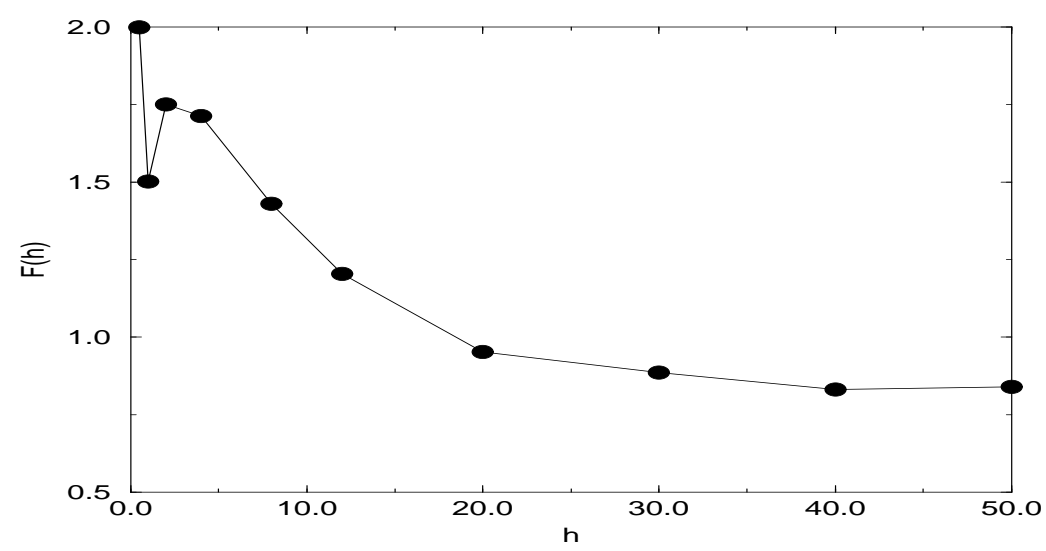

FIG. 24. $\mathcal{F}(H / m)$ vs. $H$, where $\mathcal{F}(H / m)$ is defined by the relation $\phi_{\text {eff }}(0)=(H / 2 \pi) \mathcal{F}(H / m)$ (see eqs. (9.10) and (9.11)).

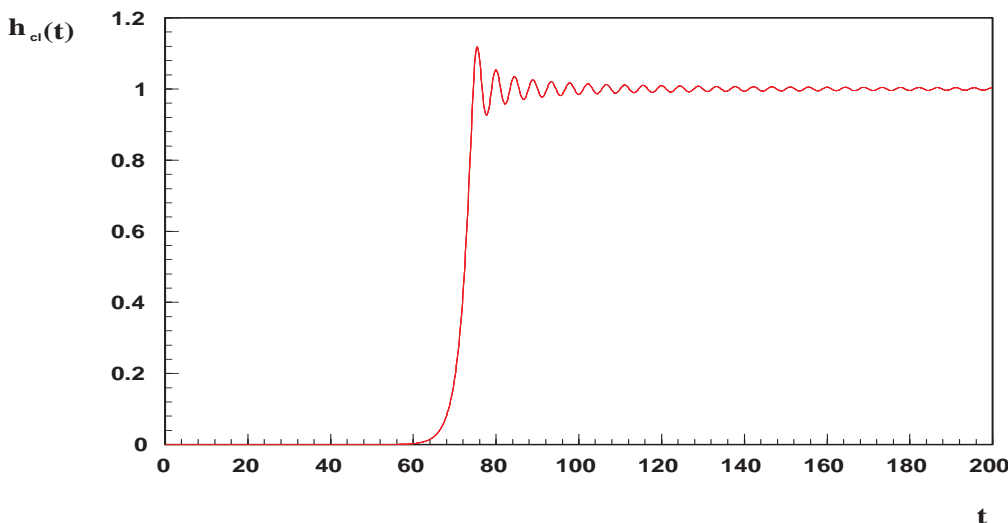

FIG. 25. $\quad \eta_{e f f}^{2}(\tau)$ vs. $\tau$, for $\eta_{\text {eff }}(0)=3.94 \times 10^{-7}, \dot{\eta}_{e f f}(0)=0.317 \eta_{e f f}(0), g=10^{-14}, h_{0}=2.0$. The initial conditions were obtained by fitting the intermediate time regime of $g \Sigma(\tau)$ in fig.19. $\eta_{\text {eff }}(\tau)$ is the solution of eq. 9.12 ) with these initial conditions.

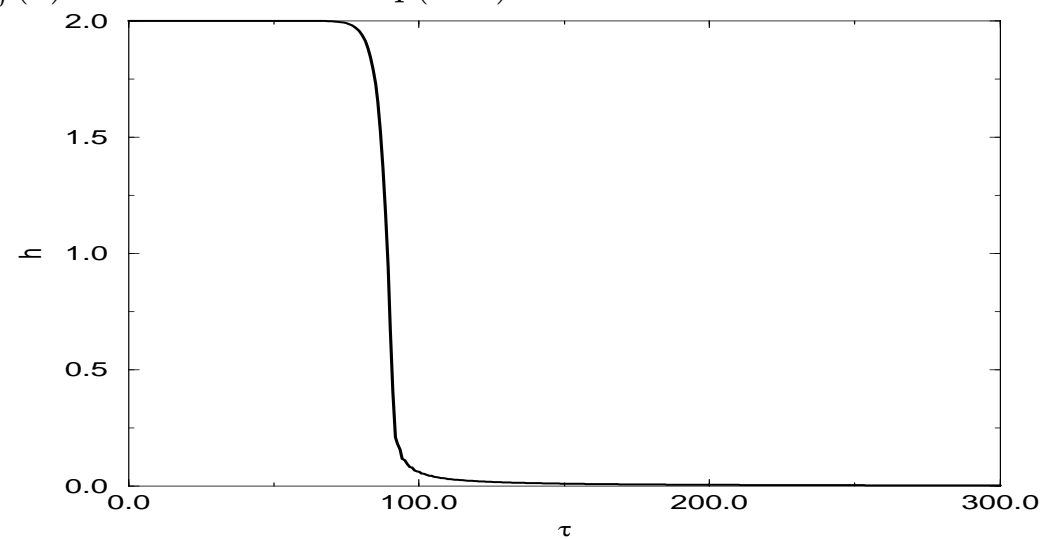

FIG. 26. $h(\tau)$ vs. $\tau$, obtained from the solution of eqs. (9.12) and (9.15) with the conditions of fig. 25 .

Therefore, in summary, the effective composite zero mode obeys

$$
\left[\frac{d^{2}}{d \tau^{2}}+3 h \frac{d}{d \tau}-1+\eta_{e f f}^{2}(\tau)\right] \eta_{e f f}(\tau)=0 ; \dot{\eta}_{e f f}(\tau=0)=\left(\nu-\frac{3}{2}\right) \eta_{e f f}(0),
$$

where $\eta_{e f f}(0) \equiv \frac{\sqrt{\lambda_{R} / 2}}{m_{R}} \phi_{e f f}(0)$ is obtained numerically for a given $h_{0}$ by fitting the interme- 
diate time behavior of $g \Sigma(\tau)$ with the growing zero mode solution. Recall that $\lambda_{R}=8 \pi^{2} g$.

Furthermore, this analysis shows that in the case $\eta=0$, the renormalized energy and pressure in this regime in which the renormalized integrals are dominated by the superhorizon modes are given by

$$
\begin{aligned}
\varepsilon_{R}(\tau) & \approx \frac{2 N m_{R}^{4}}{\lambda_{R}}\left\{\frac{1}{2} \dot{\eta}_{e f f}^{2}+\frac{1}{4}\left(-1+\eta_{e f f}^{2}\right)^{2}\right\}, \\
(p+\varepsilon)_{R} & \approx \frac{2 N m_{R}^{4}}{\lambda_{R}}\left\{\dot{\eta}_{\text {eff }}^{2}\right\},
\end{aligned}
$$

where we have neglected the contribution proportional to $1 / a^{2}(\tau)$ because it is effectively red-shifted away after just a few e-folds. We found numerically that this term is negligible after the interval of time necessary for the superhorizon modes to dominate the contribution to the integrals. Then the dynamics of the scale factor is given by

$$
h^{2}(\tau)=4 h_{0}^{2}\left\{\frac{1}{2} \dot{\eta}_{e f f}^{2}+\frac{1}{4}\left(-1+\eta_{\text {eff }}^{2}\right)^{2}\right\} .
$$

We have numerically evolved the set of effective equations (9.12, 9.15) by extracting the initial condition for the effective zero mode from the intermediate time behavior of $g \Sigma(\tau)$. We found a remarkable agreement between the evolution of $\eta_{\text {eff }}^{2}$ and $g \Sigma(\tau)$ and between the dynamics of the scale factor in terms of the evolution of $\eta_{\text {eff }}(\tau)$, and the full dynamics of the scale factor and quantum fluctuations within our numerical accuracy. Figs. 25 and 26 show the evolution of $\eta_{\text {eff }}^{2}(\tau)$ and $h(\tau)$ respectively from the classical evolution eqs. (9.12) and (9.15) using the initial condition $\eta_{\text {eff }}(0)$ extracted from the exponential fit of $g \Sigma(\tau)$ in the intermediate regime. These figures should be compared to figs. 19 and 20 . We have also numerically compared $p / \varepsilon$ given solely by the dynamics of the effective zero mode and it is again numerically indistinguishable from that obtained with the full evolution of the mode functions.

This is one of the main results of our work. In summary: the modes that become superhorizon sized and grow through the spinodal instabilities assemble themselves into an effective composite zero mode a few e-folds after the beginning of inflation. This effective zero mode drives the dynamics of the FRW scale factor, terminating inflation when the nonlinearities become important. In terms of the underlying fluctuations, the spinodal growth of superhorizon modes gives a non-perturbatively large contribution to the energy momentum tensor that drives the dynamics of the scale factor. Inflation terminates when the mean square root fluctuation probes the equilibrium minima of the tree level potential.

This phenomenon of zero mode assembly, i.e. the 'classicalization' of quantum mechanical fluctuations that grow after horizon crossing is very similar to the interpretation of 'decoherence without decoherence' of Starobinsky and Polarski [43].

The extension of this analysis to the case for which $\eta(0) \neq 0$ is straightforward. Since both $\eta(\tau)$ and $\sqrt{g \Sigma(\tau)}=\left|C_{0}\right| f_{0}(\tau)$ obey the equation for the zero mode, eq.(5.18), it is clear that we can generalize our definition of the effective zero mode to be

$$
\eta_{e f f}(\tau) \equiv \sqrt{\eta^{2}(\tau)+g \Sigma(\tau)} .
$$

which obeys the equation of motion of a classical zero mode: 


$$
\left[\frac{d^{2}}{d \tau^{2}}+3 h \frac{d}{d \tau}-1+\eta_{e f f}(\tau)^{2}\right] \eta_{e f f}(\tau)=0
$$

If this effective zero mode is to drive the FRW expansion, then the additional condition

$$
\dot{\eta}^{2} f_{0}^{2}-2 \eta \dot{\eta} f_{0} \dot{f}_{0}+\eta^{2} \dot{f}_{0}^{2}=0
$$

must also be satisfied. One can easily show that this relation is indeed satisfied if the mode functions factorize as in (9.5) and if the integrals (9.6) - (9.8) are dominated by the contributions of the superhorizon mode functions. This leads to the conclusion that the gravitational dynamics is given by eqs. (9.13) - (9.15) with $\eta_{\text {eff }}(\tau)$ defined by $(9.16)$.

We see that in all cases, the full large $N$ quantum dynamics in these models of inflationary phase transitions is well approximated by the equivalent dynamics of a homogeneous, classical scalar field with initial conditions on the effective field $\eta_{\text {eff }}(0) \geq \sqrt{g} h_{0} \mathcal{F}\left(h_{0}\right)$. We have verified these results numerically for the field and scale factor dynamics, finding that the effective classical dynamics reproduces the results of the full dynamics to within our numerical accuracy. We have also checked numerically that the estimate for the classical to quantum crossover given by eq. (9.4) is quantitatively correct. Thus in the classical case in which $\eta(0) \gg \sqrt{g} h_{0}$ we find that $\eta_{e f f}(\tau)=\eta(\tau)$, whereas in the opposite, quantum case $\eta_{\text {eff }}(\tau)=\sqrt{g \Sigma(\tau)}$.

This remarkable feature of zero mode assembly of long-wavelength, spinodally unstable modes is a consequence of the presence of the horizon. It also explains why, despite the fact that asymptotically the fluctuations sample the broken symmetry state, the equation of state is that of matter. Since the excitations in the broken symmetry state are massless Goldstone bosons one would expect radiation domination. However, the assembly phenomenon, i.e. the redshifting of the wave vectors, makes these modes behave exactly like zero momentum modes that give an equation of state of matter (upon averaging over the small oscillations around the minimum).

Subhorizon modes at the end of inflation with $q>h_{0} e^{h_{0} \tau_{s}}$ do not participate in the zero mode assembly. The behavior of such modes do depend on $q$ after the end of inflation. Notice that these modes have extremely large comoving $q$ since $h_{0} e^{h_{0} \tau_{s}} \geq 10^{26}$. As discussed in sec. VII such modes decrease with time after inflation as $\sim 1 / a(\tau)$ [14.

\section{Making sense of small fluctuations:}

Having recognized the effective classical variable that can be interpreted as the component of the field that drives the FRW background and rolls down the classical potential hill, we want to recognize unambiguously the small fluctuations. We have argued above that after horizon crossing, all of the mode functions evolve proportionally to the zero mode, and the question arises: which modes are assembled into the effective zero mode whose dynamics drives the evolution of the FRW scale factor and which modes are treated as perturbations? In principle every $k \neq 0$ mode provides some spatial inhomogeneity, and assembling these into an effective homogeneous zero mode seems in principle to do away with the very inhomogeneities that one wants to study. However, scales of cosmological importance today first crossed the horizon during the last 60 or so e-folds of inflation. Recently Grishchuk 
[44] has argued that the sensitivity of the measurements of $\Delta T / T$ probe inhomogeneities on scales $\approx 500$ times the size of the present horizon. Therefore scales that are larger than these and that have first crossed the horizon much earlier than the last 60 e-folds of inflation are unobservable today and can be treated as an effective homogeneous component, whereas the scales that can be probed experimentally via the CMB inhomogeneities today must be treated separately as part of the inhomogeneous perturbations of the CMB.

Thus a consistent description of the dynamics in terms of an effective zero mode plus 'small' quantum fluctuations can be given provided the following requirements are met: a) the total number of e-folds $N_{e} \gg 60$, b) all the modes that have crossed the horizon before the last 60-65 e-folds are assembled into an effective classical zero mode via $\phi_{\text {eff }}(t)=$ $\left[\phi_{0}^{2}(t)+\left\langle\psi^{2}(\vec{x}, t)\right\rangle_{R}\right]^{\frac{1}{2}}$, c) the modes that cross the horizon during the last 60-65 e-folds are accounted as 'small' perturbations. The reason for the requirement a) is that in the separation $\phi(\vec{x}, t)=\phi_{\text {eff }}(t)+\delta \phi(\vec{x}, t)$ one requires that $\delta \phi(\vec{x}, t) / \phi_{\text {eff }}(t) \ll 1$. As argued above, after the modes cross the horizon, the ratio of amplitudes of the mode functions remains constant and given by $e^{\left(\nu-\frac{3}{2}\right) \Delta N}$ with $\Delta N$ being the number of e-folds between the crossing of the smaller $k$ and the crossing of the larger $k$. Then for $\delta \phi(\vec{x}, t)$ to be much smaller than the effective zero mode, it must be that the Fourier components of $\delta \phi$ correspond to very large $k$ 's at the beginning of inflation, so that the effective zero mode can grow for a long time before the components of $\delta \phi$ begin to grow under the spinodal instabilities. In fact requirement a) is not very severe; in the figs.(19-23) we have taken $h_{0}=2.0$ which is a very moderate value and yet for $g=10^{-12}$ the inflationary stage lasts for well over 100 e-folds, and as argued above, the larger $h_{0}$ for fixed $g$, the longer is the inflationary stage. Therefore under this set of conditions, the classical dynamics of the effective zero mode $\phi_{\text {eff }}(t)$ drives the FRW background, whereas the inhomogeneous fluctuations $\delta \phi(\vec{x}, t)$, which are made up of Fourier components with wavelengths that are much smaller than the horizon at the beginning of inflation and that cross the horizon during the last 60 e-folds, provide the inhomogeneities that seed density perturbations.

\section{E. Scalar Metric Perturbations:}

Having identified the effective zero mode and the 'small perturbations', we are now in position to provide an estimate for the amplitude and spectrum of scalar metric perturbations. We use the clear formulation in ref. 45] in terms of gauge invariant variables. In particular we focus on the dynamics of the Bardeen potential [46], which in longitudinal gauge is identified with the Newtonian potential. The equation of motion for the Fourier components (in terms of comoving wavevectors) for this variable in terms of the effective zero mode is 45

$$
\ddot{\Phi}_{k}+\left[H(t)-2 \frac{\ddot{\phi}_{e f f}(t)}{\dot{\phi}_{e f f}(t)}\right] \dot{\Phi}_{k}+\left[\frac{k^{2}}{a^{2}(t)}+2\left(\dot{H}(t)-H(t) \frac{\ddot{\phi}_{e f f}(t)}{\dot{\phi}_{e f f}(t)}\right)\right] \Phi_{k}=0 .
$$

We are interested in determining the dynamics of $\Phi_{k}$ for those wavevectors that cross the horizon during the last 60 e-folds before the end of inflation. During the inflationary stage the numerical analysis yields to a very good approximation

$$
H(t) \approx H_{0} ; \phi_{\text {eff }}(t)=\phi_{e f f}(0) e^{\left(\nu-\frac{3}{2}\right) H_{0} t},
$$


where $H_{0}$ is the value of the Hubble constant during inflation, leading to

$$
\Phi_{k}(t)=e^{(\nu-2) H_{0} t}\left[a_{k} H_{\beta}^{(1)}\left(\frac{k e^{-H_{0} t}}{H_{0}}\right)+b_{k} H_{\beta}^{(2)}\left(\frac{k e^{-H_{0} t}}{H_{0}}\right)\right] ; \beta=\nu-1 .
$$

The coefficients $a_{k}, b_{k}$ are determined by the initial conditions.

Since we are interested in the wavevectors that cross the horizon during the last 60 e-folds, the consistency for the zero mode assembly and the interpretation of 'small perturbations' requires that there must be many e-folds before the last 60 . We are then considering wavevectors that were deep inside the horizon at the onset of inflation. $\Phi_{k}(t)$ is related to the canonical 'velocity field' that determines scalar perturbations of the metric and which is quantized with Bunch-Davies initial conditions for the large $k$-mode functions. The relation between $\Phi_{k}$ and $v$ and the initial conditions on $v$ lead at once to a determination of the coefficients $a_{k}$ and $b_{k}$ for $k>>H_{0}$

$$
a_{k}=-\frac{3}{2}\left[\frac{8 \pi}{3 M_{P l}^{2}}\right] \dot{\phi}_{e f f}(0) \sqrt{\frac{\pi}{2 H_{0}}} \frac{1}{k} \quad ; \quad b_{k}=0 .
$$

Thus we find that the amplitude of scalar metric perturbations after horizon crossing is given by

$$
\left|\delta_{k}(t)\right|=k^{\frac{3}{2}}\left|\Phi_{k}(t)\right| \approx \frac{3}{2}\left[\frac{8 \sqrt{\pi}}{3 M_{P l}^{2}}\right] \dot{\phi}_{e f f}(0)\left(\frac{2 H_{0}}{k}\right)^{\nu-\frac{3}{2}} e^{(2 \nu-3) H_{0} t} .
$$

The power spectrum per logarithmic $k$ interval is given by $\left|\delta_{k}(t)\right|^{2}$. The time dependence of $\left|\delta_{k}(t)\right|$ displays the unstable growth associated with the spinodal instabilities of superhorizon modes and is a hallmark of the phase transition. This time dependence can be also understood from the constraint equation that relates the Bardeen potential to the gauge invariant field fluctuations [45], which in longitudinal gauge are identified with $\delta \phi(\vec{x}, t)$. The constraint equation and the evolution equations for the gauge invariant scalar field fluctuations are [45]

$$
\begin{gathered}
\frac{d}{d t}\left(a \Phi_{k}\right)=\frac{4 \pi}{M_{P l}^{2}} a \delta \phi_{k}^{g i} \dot{\phi}_{0} \\
{\left[\frac{d^{2}}{d t^{2}}+3 H \frac{d}{d t}+\frac{k^{2}}{a^{2}}+\mathcal{M}^{2}\right] \delta \phi_{k}^{g i}-4 \dot{\phi}_{e f f} \dot{\Phi}_{k}+2 V^{\prime}\left(\phi_{e f f}\right) \Phi_{k}=0 .}
\end{gathered}
$$

Since the right hand side of $(9.24)$ is proportional to $\dot{\phi}_{\text {eff }} / M_{P l}^{2} \ll 1$ during the inflationary epoch in this model, we can neglect the terms proportional to $\dot{\Phi}_{k}$ and $\Phi_{k}$ on the left hand side of (9.25), in which case the equation for the gauge invariant scalar field fluctuation is the same as for the mode functions. In fact, since $\delta \phi_{k}^{g i}$ is gauge invariant we can evaluate it in the longitudinal gauge wherein it is identified with the mode functions $f_{k}(t)$. Then absorbing a constant of integration in the initial conditions for the Bardeen variable, we find

$$
\Phi_{k}(t) \approx \frac{4 \pi}{M_{P l}^{2} a(t)} \int_{t_{o}}^{t} a\left(t^{\prime}\right) \phi_{e f f}\left(t^{\prime}\right) f_{k}\left(t^{\prime}\right) d t^{\prime}+\mathcal{O}\left(\frac{1}{M_{P l}^{4}}\right),
$$


and using that $\phi(t) \propto e^{(\nu-3 / 2) H_{0} t}$ and that after horizon crossing $f_{k}(t) \propto e^{(\nu-3 / 2) H_{0} t}$, one obtains at once the time dependence of the Bardeen variable after horizon crossing. In particular the time dependence is found to be $\propto e^{(2 \nu-3) H_{0} t}$. It is then clear that the time dependence is a reflection of the spinodal (unstable) growth of the superhorizon field fluctuations.

To obtain the amplitude and spectrum of density perturbations at second horizon crossing we use the conservation law associated with the gauge invariant variable 445]

$$
\xi_{k}=\frac{2}{3} \frac{\frac{\dot{\Phi}_{k}}{H}+\Phi_{k}}{1+p / \varepsilon}+\Phi_{k} ; \quad \dot{\xi}_{k}=0,
$$

which is valid after horizon crossing of the mode with wavevector $k$. Although this conservation law is an exact statement of superhorizon mode solutions of eq.(9.19), we have obtained solutions assuming that during the inflationary stage $H$ is constant and have neglected the $\dot{H}$ term in Eq. (9.19). Since during the inflationary stage,

$$
\dot{H}(t)=-\frac{4 \pi}{M_{P l}^{2}} \dot{\phi}_{e f f}^{2}(t) \propto H_{0}^{2}\left(\frac{d \eta_{e f f}(\tau)}{d \tau}\right)^{2} \ll H_{0}^{2}
$$

and $\ddot{\phi} / \dot{\phi} \approx H_{0}$, the above approximation is justified. We then see that $\phi_{\text {eff }}^{2}(t) \propto e^{(2 \nu-3) H_{0} t}$ which is the same time dependence as that of $\Phi_{k}(t)$. Thus the term proportional to $1 /(1+$ $p / \varepsilon)$ in Eq. (9.27) is indeed constant in time after horizon crossing. On the other hand, the term that does not have this denominator evolves in time but is of order $(1+p / \varepsilon)=$ $-2 \dot{H} / 3 H^{2} \ll 1$ with respect to the constant term and therefore can be neglected. Thus, we confirm that the variable $\xi$ is conserved up to the small term proportional to $(1+p / \varepsilon) \Phi_{k}$ which is negligible during the inflationary stage. This small time dependence is consistent with the fact that we neglected the $\dot{H}$ term in the equation of motion for $\Phi_{k}(t)$. The validity of the conservation law has been recently studied and confirmed in different contexts [47]. Notice that we do not have to assume that $\dot{\Phi}_{k}$ vanishes, which in fact does not occur.

However, upon second horizon crossing it is straightforward to see that $\dot{\Phi}_{k}\left(t_{f}\right) \approx 0$. The reason for this assertion can be seen as follows: eq.(9.25) shows that at long times, when the effective zero mode is oscillating around the minimum of the potential with a very small amplitude and when the time dependence of the fluctuations has saturated (see fig.3), $\Phi_{k}$ will redshift as $\approx 1 / a(t)$ [14] and its derivative becomes extremely small.

Using this conservation law, assuming matter domination at second horizon crossing, and $\dot{\Phi}_{k}\left(t_{f}\right) \approx 0$ [45, we find

$$
\left|\delta_{k}\left(t_{f}\right)\right|=\frac{12 \Gamma(\nu) \sqrt{\pi}}{5\left(\nu-\frac{3}{2}\right) \mathcal{F}\left(H_{0} / m\right)}\left(\frac{2 H_{0}}{k}\right)^{\nu-\frac{3}{2}},
$$

where $\mathcal{F}\left(H_{0} / m\right)$ determines the initial amplitude of the effective zero mode (9.11). We can now read the power spectrum per logarithmic $k$ interval

$$
\mathcal{P}_{s}(k)=\left|\delta_{k}\right|^{2} \propto k^{-2\left(\nu-\frac{3}{2}\right)} .
$$

leading to the index for scalar density perturbations 


$$
n_{s}=1-2\left(\nu-\frac{3}{2}\right)
$$

For $H_{0} / m \gg 1$, we can expand $\nu-3 / 2$ as a series in $m^{2} / H_{0}^{2}$ in eq.(9.29). Given that the comoving wavenumber of the mode which crosses the horizon $n$ e-folds before the end of inflation is $k=H_{0} e^{\left(N_{e}-n\right)}$ where $N_{e}$ is given by $(9.3)$, we arrive at the following expression for the amplitude of fluctuations on the scale corresponding to $n$ in terms of the De Sitter Hubble constant and the coupling $\lambda=8 \pi^{2} g$ :

$$
\left|\delta_{n}\left(t_{f}\right)\right| \simeq \frac{9 H^{3}}{5 \sqrt{2} m^{3}}\left(2 e^{n}\right)^{m^{2} / 3 H_{0}^{2}} \sqrt{\lambda}\left[1+\frac{2 m^{2}}{3 H_{0}^{2}}\left(\frac{7}{6}-\ln 2-\frac{\gamma}{2}\right)+\mathcal{O}\left(\frac{m^{4}}{H_{0}^{4}}\right)\right] .
$$

Here, $\gamma$ is Euler's constant. Note the explicit dependence of the amplitude of density perturbations on $\sqrt{g}$. For $n \approx 60$, the factor $\exp \left(n m^{2} / 3 H_{0}^{2}\right)$ is $\mathcal{O}(100)$ for $H_{0} / m=2$, while it is $\mathcal{O}(1)$ for $H_{0} / m \geq 4$. Notice that for $H_{0} / m$ large, the amplitude increases approximately as $\left(H_{0} / m\right)^{3}$, which will place strong restrictions on $g$ in such models.

We remark that we have not included the small corrections to the dynamics of the effective zero mode and the scale factor arising from the non-linearities. We have found numerically that these nonlinearities are only significant for the modes that cross about 60 e-folds before the end of inflation for values of the Hubble parameter $H_{0} / m_{R}>5$. The effect of these non-linearities in the large $N$ limit is to slow somewhat the exponential growth of these modes, with the result of shifting the power spectrum closer to an exact HarrisonZeldovich spectrum with $n_{s}=1$. Since for $H_{0} / m_{R}>5$ the power spectrum given by (9.31) differs from one by at most a few percent, the effects of the non-linearities are expected to be observationally unimportant. The spectrum given by (9.29) is similar to that obtained in references [3, 38 although the amplitude differs from that obtained there. In addition, we do not assume slow roll for which $\left(\nu-\frac{3}{2}\right) \ll 1$, although this would be the case if $N_{e} \gg 60$.

We emphasize an important feature of the spectrum: it has more power at long wavelengths because $\nu-3 / 2>0$. This is recognized to be a consequence of the spinodal instabilities that result in the growth of long wavelength modes and therefore in more power for these modes. This seems to be a robust prediction of new inflationary scenarios in which the potential has negative second derivative in the region of field space that produces inflation.

It is at this stage that we recognize the consistency of our approach for separating the composite effective zero mode from the small fluctuations. We have argued above that many more than 60 e-folds are required for consistency, and that the small fluctuations correspond to those modes that cross the horizon during the last 60 e-folds of the inflationary stage. For these modes $H_{0} / k=e^{-H_{0} t^{*}(k)}$ where $t^{*}(k)$ is the time since the beginning of inflation of horizon crossing of the mode with wavevector $k$. The scale that corresponds to the Hubble radius today $\lambda_{0}=2 \pi / k_{0}$ is the first to cross during the last 60 or so e-folds before the end of inflation. Smaller scales today will correspond to $k>k_{0}$ at the onset of inflation since they will cross the first horizon later and therefore will reenter earlier. The bound on $\left|\delta_{k_{0}}\right| \propto \Delta T / T \leq 10^{-5}$ on these scales provides a lower bound on the number of e-folds required for these type of models to be consistent:

$$
N_{e}>60+\frac{12}{\nu-\frac{3}{2}}-\frac{\ln \left(\nu-\frac{3}{2}\right)}{\nu-\frac{3}{2}},
$$


where we have written the total number of e-folds as $N_{e}=H_{0} t^{*}\left(k_{0}\right)+60$. This in turn can be translated into a bound on the coupling constant using the estimate given by eq.(9.3).

The four year COBE DMR Sky Map [48] gives $n \approx 1.2 \pm 0.3$ thus providing an upper bound on $\nu$

$$
0 \leq \nu-\frac{3}{2} \leq 0.05
$$

corresponding to $h_{0} \geq 2.6$. We then find that these values of $h_{0}$ and $\lambda \approx 10^{-12}-10^{-14}$ provide sufficient e-folds to satisfy the constraint for scalar density perturbations.

\section{F. Tensor Metric Perturbations:}

The scalar field does not couple to the tensor (gravitational wave) modes directly, and the tensor perturbations are gauge invariant from the beginning. Their dynamical evolution is completely determined by the dynamics of the scale factor 445,49. Having established

numerically that the inflationary epoch is characterized by $\dot{H} / H_{0}^{2} \ll 1$ and that scales of cosmological interest cross the horizon during the stage in which this approximation is excellent, we can just borrow the known result for the power spectrum of gravitational waves produced during inflation extrapolated to the matter era 45,49

$$
\mathcal{P}_{T}(k) \approx \frac{H_{0}^{2}}{M_{P l}^{2}} k^{0} .
$$

Thus the spectrum to this order is scale invariant (Harrison-Zeldovich) with an amplitude of the order $m^{4} / \lambda M_{P l}^{4}$. Then, for values of $m \approx 10^{12}-10^{14} \mathrm{Gev}$ and $\lambda \approx 10^{-12}-10^{-14}$ one finds that the amplitude is $\leq 10^{-10}$ which is much smaller than the amplitude of scalar density perturbations. As usual the amplification of scalar perturbations is a consequence of the equation of state during the inflationary epoch.

\section{G. Contact with the Reconstruction Program:}

The program of reconstruction of the inflationary potential seeks to establish a relationship between features of the inflationary scalar potential and the spectrum of scalar and tensor perturbations. This program, in combination with measurements of scalar and tensor components either from refined measurements of temperature inhomogeneities of the CMB or through galaxy correlation functions will then offer a glimpse of the possible realization of the inflation [50,51. Such a reconstruction program is based on the slow roll approximation and the spectral index of scalar and tensor perturbations are obtained in a perturbative expansion in the slow roll parameters 50,51

$$
\begin{aligned}
\epsilon(\phi) & =\frac{\frac{3}{2} \dot{\phi}^{2}}{\frac{\dot{\phi}^{2}}{2}+V(\phi)}, \\
\eta(\phi) & =-\frac{\ddot{\phi}}{H \dot{\phi}} .
\end{aligned}
$$


We can make contact with the reconstruction program by identifying $\phi$ above with our $\phi_{\text {eff }}$ after the first few e-folds of inflation needed to assemble the effective zero mode from the quantum fluctuations. We have numerically established that for the weak scalar coupling required for the consistency of these models, the cosmologically interesting scales cross the horizon during the epoch in which $H \approx H_{0} ; \dot{\phi}_{\text {eff }} \approx(\nu-3 / 2) H_{0} \phi_{\text {eff }} ; V \approx m_{R}^{4} / \lambda \gg \dot{\phi}_{\text {eff }}^{2}$. In this case we find

$$
\eta\left(\phi_{e f f}\right)=-\left(\nu-\frac{3}{2}\right) ; \epsilon\left(\phi_{e f f}\right) \approx \mathcal{O}(\lambda) \ll \eta\left(\phi_{e f f}\right) .
$$

With these identifications, and in the notation of [50,51 the reconstruction program predicts the index for scalar density perturbations $n_{s}$ given by

$$
n_{s}-1=-2\left(\nu-\frac{3}{2}\right)+\mathcal{O}(\lambda)
$$

which coincides with the index for the power spectrum per logarithmic interval $\left|\delta_{k}\right|^{2}$ with $\left|\delta_{k}\right|$ given by eq.(9.29). We must note however that our treatment did not assume slow roll

for which $\left(\nu-\frac{3}{2}\right) \ll 1$. Our self-consistent, non-perturbative study of the dynamics plus the underlying requirements for the identification of a composite operator acting as an effective zero mode, validates the reconstruction program in weakly coupled new inflationary models.

\section{DECOHERENCE: QUANTUM TO CLASSICAL TRANSITION DURING INFLATION}

An important aspect of cosmological perturbations is that they are of quantum origin but eventually they become classical as they are responsible for the small classical metric perturbations. This quantum to classical crossover is associated with a decoherence process and has received much attention [43,52].

In this section we study the quantum to classical transition of superhorizon modes for the Bardeen variable by relating these to the field mode functions through eq.(9.26) and analyzing the full time evolution of the density matrix of the matter field. Eq.(9.26) establishes that in the models under consideration the classicality of the Bardeen variable is determined by the classicality of the scalar field modes.

In the situation under consideration, long-wavelength field modes become spinodally unstable and grow exponentially after horizon crossing. The factorization (9.1) results in the phases of these modes 'freezing out'. This feature and the growth in amplitude entail that these modes become classical. Eq.(9.26) in turn implies that these features also apply to the superhorizon modes of the Bardeen potential.

Therefore we can address the quantum to classical transition of the Bardeen variable (gravitational potential) by analyzing the evolution of the density matrix for the matter field.

To make contact with previous work 43,52 we choose to study the evolution of the field density matrix in conformal time, although the same features will be displayed in comoving time.

In the large $N$ or Hartree (also in the self-consistent one-loop) approximation, the density matrix is Gaussian, and defined by a normalization factor, a complex covariance that 
determines the diagonal matrix elements, and a real covariance that determines the mixing in the Schrödinger representation as discussed in ref. [13 (and references therein).

That is, the density matrix takes the form

$$
\begin{aligned}
\rho[\Phi, \tilde{\Phi}, \mathcal{T}]= & \prod_{\vec{k}} \mathcal{N}_{k}(\mathcal{T}) \exp \left\{-\frac{1}{2} A_{k}(\mathcal{T}) \vec{\eta}_{\vec{k}}(\mathcal{T}) \cdot \vec{\eta}_{-\vec{k}}(\mathcal{T})-\frac{1}{2} A_{k}^{*}(\mathcal{T}) \tilde{\vec{\eta}}_{\vec{k}}(\mathcal{T}) \cdot \tilde{\vec{\eta}}_{-\vec{k}}(\mathcal{T})\right. \\
& \left.-B_{k}(\mathcal{T}) \vec{\eta}_{\vec{k}}(\mathcal{T}) \cdot \tilde{\vec{\eta}}_{-\vec{k}}(\mathcal{T})+i \vec{\pi}_{\vec{k}}(\mathcal{T}) \cdot\left(\vec{\eta}_{-\vec{k}}(\mathcal{T})-\tilde{\vec{\eta}}_{-\vec{k}}(\mathcal{T})\right)\right\} ; \\
\vec{\eta}_{\vec{k}}(\mathcal{T})= & \vec{\chi}_{\vec{k}}(\mathcal{T})-\chi_{0}(\mathcal{T}) \delta_{i, 1} \delta(\vec{k}) ; \tilde{\vec{\eta}}_{k}(\mathcal{T})=\tilde{\vec{\chi}}_{k}(\mathcal{T})-\chi_{0}(\mathcal{T}) \delta_{i, 1} \delta(\vec{k}) .
\end{aligned}
$$

$\vec{\pi}_{\vec{k}}(\mathcal{T})$ is the Fourier transform of $\Pi_{\chi}(\mathcal{T}, \vec{x})$. This form of the density matrix is dictated by the hermiticity condition

$$
\rho[\Phi, \tilde{\Phi}, \mathcal{T}]=\rho^{*}[\tilde{\Phi}, \Phi, \mathcal{T}]
$$

as a result of this, $B_{k}(\mathcal{T})$ is real. The kernel $B_{k}(\mathcal{T})$ determines the amount of 'mixing' in the density matrix since if $B_{k}=0$, the density matrix corresponds to a pure state because it factorizes into a wave functional depending only on $\Phi(\cdot)$ times its complex conjugate taken at $\tilde{\Phi}(\cdot)$. This is the case under consideration, since the initial conditions correspond to a pure state and under time evolution the density matrix remains that of a pure state [13].

In conformal time quantization the evolution of the density matrix is via the conformal time Hamiltonian (6.5). The evolution equations for the covariances (given by equation (2.20) in [13]; see also equation (2.44) of [13]) are obtained from those given in ref. [13] by setting $a(t)=1$ and using the frequencies $\omega_{k}^{2}(\mathcal{T})=k^{2}+\mathcal{M}^{2}(\mathcal{T})$. In particular, by setting

$$
A_{k}(\mathcal{T})=-i \frac{F_{k}^{* *}(\mathcal{T})}{F_{k}^{*}(\mathcal{T})}
$$

More explicitly [13,

$$
\begin{aligned}
& \mathcal{N}_{k}(\mathcal{T})=\mathcal{N}_{k}\left(\mathcal{T}_{0}\right) \exp \left[\int_{\mathcal{T}_{0}}^{\mathcal{T}} A_{I k}\left(\mathcal{T}^{\prime}\right) d \mathcal{T}^{\prime}\right]=\frac{\mathcal{N}_{k}\left(\mathcal{T}_{0}\right)}{\sqrt{\omega_{k}\left(\mathcal{T}_{o}\right)}\left|F_{k}(\mathcal{T})\right|} \\
& A_{I k}(\mathcal{T})=-\frac{d}{d \mathcal{T}} \log \left|F_{k}(\mathcal{T})\right|=-\dot{a}(t)-a(t) \frac{d}{d t} \log \left|f_{k}(t)\right| \\
& A_{R k}(\mathcal{T})=\frac{1}{\left|F_{k}(\mathcal{T})\right|^{2}}=\frac{1}{a(t)^{2}\left|f_{k}(t)\right|^{2}}, B_{k}(\mathcal{T}) \equiv 0
\end{aligned}
$$

where $A_{R k}$ and $A_{I k}$ are respectively the real and imaginary parts of $A_{k}$ and we have used the value of the Wronskian (6.12) in evaluating (10.3).

The coefficients $A_{k}(\mathcal{T})$ and $\mathcal{N}_{k}(\mathcal{T})$ in the gaussian density matrix (10.1) are completely determined by the conformal mode functions $F_{k}(\mathcal{T})$ (or alternatively the comoving time mode functions $\left.f_{k}(t)\right)$.

Let us study the time behavior of these coefficients. During inflation, $a(t) \approx e^{h_{0} t}$, and the mode functions factorize after horizon crossing, and superhorizon modes grow in cosmic time as in Eq. (9.1):

$$
a^{2}(t)\left|f_{k}(t)\right|^{2} \approx \frac{1}{\mathcal{D}_{k}} e^{(2 \nu-1) h_{0} t}
$$

where the coefficient $\mathcal{D}_{k}$ can be read from eq. (9.1). 
We emphasize that this is a result of the full evolution as displayed from the numerical solution in fig.21. These mode functions encode all of the self-consistent and non-perturbative features of the dynamics. This should be contrasted with other studies in which typically free field modes in a background metric are used.

Inserting this expression in eqs.(10.3) yields,

$$
\begin{aligned}
& A_{I k}(\mathcal{T}) \stackrel{t \rightarrow \infty}{=}-h_{0} e^{h_{0} t}\left(\nu-\frac{1}{2}\right)+\mathcal{O}\left(e^{-h_{0} t}\right), \\
& A_{R k}(\mathcal{T}) \stackrel{t \rightarrow \infty}{=} \mathcal{D}_{k} e^{-(2 \nu-1) h_{0} t}
\end{aligned}
$$

Since $\nu-\frac{1}{2}>1$, we see that the imaginary part of the covariance $A_{I k}(\mathcal{T})$ grows very fast. Hence, the off-diagonal elements of $\rho[\Phi, \tilde{\Phi}, \mathcal{T}]$ oscillate wildly after a few e-folds of inflation. In particular their contribution to expectation values of operators will be washed out. That is, we quickly reach a classical regime where only the diagonal part of the density matrix is relevant:

$$
\rho[\Phi, \Phi, \mathcal{T}]=\prod_{\vec{k}} \mathcal{N}_{k}(\mathcal{T}) \exp \left\{-A_{R k}(\mathcal{T}) \eta_{\vec{k}}(\mathcal{T}) \eta_{-\vec{k}}(\mathcal{T})\right\}
$$

The real part of the covariance $A_{R k}(\mathcal{T})$ (as well as any non-zero mixing kernel $B_{k}(\mathcal{T})$ [13]) decreases as $e^{-(2 \nu-1) h_{0} t}$. Therefore, characteristic field configurations $\eta_{\vec{k}}$ are very large (of order $\left.e^{\left(\nu-\frac{1}{2}\right) h_{0} t}\right)$. Therefore configurations with field amplitudes up to $\mathcal{O}\left(e^{\left(\nu-\frac{1}{2}\right) h_{0} t}\right)$ will have a substantial probability of occurring and being represented in the density matrix.

Notice that $\chi \sim e^{\left(\nu-\frac{1}{2}\right) h_{0} t}$ corresponds to field configurations $\Phi$ with amplitudes of order $e^{\left(\nu-\frac{3}{2}\right) h_{0} t}$ [see eq. 6.2)]. It is the fact that $\nu-\frac{3}{2}>0$ which in this situation is responsible for the 'classicalization', which is seen to be a consequence of the spinodal growth of longwavelength fluctuations.

The equal-time field correlator is given by

$$
\begin{aligned}
\left\langle\bar{\chi}(\vec{x}, \mathcal{T}) \bar{\chi}\left(\vec{x}^{\prime}, \mathcal{T}\right)\right\rangle & =\int \frac{d^{3} k}{2(2 \pi)^{3}}\left|F_{k}(\mathcal{T})\right|^{2} e^{i \vec{k} \cdot\left(\vec{x}-\vec{x}^{\prime}\right)} \\
& =a(t)^{2} \int \frac{d^{3} k}{2(2 \pi)^{3}}\left|f_{k}(t)\right|^{2} e^{i \vec{k} \cdot\left(\vec{x}-\vec{x}^{\prime}\right)}
\end{aligned}
$$

and is seen to be dominated by the superhorizon mode functions and to grow as $e^{(2 \nu-1) h_{0} t}$, whereas the field commutators remain fixed showing the emergence of a classical behavior. As a result we obtain

$$
\left\langle\bar{\chi}(\vec{x}, \mathcal{T}) \bar{\chi}\left(\vec{x}^{\prime}, \mathcal{T}\right)\right\rangle \propto a^{2}(t) \phi_{\text {eff }}(t) \phi_{\text {eff }}\left(t^{\prime}\right) G\left(\left|\vec{x}-\vec{x}^{\prime}\right|\right)+\text { small }
$$

where $G\left(\left|\vec{x}-\vec{x}^{\prime}\right|\right)$ falls off exponentially for distances larger than the horizon 15 and 'small' refers to terms that are smaller in magnitude. This factorization of the correlation functions is another indication of classicality.

Therefore, it is possible to describe the physics by using classical field theory. More precisely, one can use a classical statistical (or stochastic) field theory described by the functional probability distribution (10.3). 
These results generalize the decoherence treatment given in ref. [53] for a free massless field in pure quantum states to the case of interacting fields with broken symmetry. Note that the formal decoherence or classicalization in the density matrix appears after the modes with wave vector $k$ become superhorizon sized i.e. when the factorization of the mode functions becomes effective.

\section{CONCLUSIONS}

Since there are a number of articles in the literature treating the problem of preheating, it is useful to review the unique features of the present work. First, we have treated the problem dynamically, without using the effective potential (an equilibrium construct) to determine the evolution. Second, we have provided consistent non-perturbative calculations of the evolution to bring out some of the most relevant aspects of the late time behavior. In particular, we found that the quantum backreaction naturally inhibits catastrophic growth of fluctuations and provides a smooth transition to the late time regime in which the quantum fluctuations decay as the zero mode approaches its asymptotic state. Third, the dynamics studied obeys the constraint of covariant conservation of the energy momentum tensor.

The Hartree approximation and the large $N$ limit are physically inequivalent. The Hartree approximation considers a single field with self-coupling $g$ while in the large $N$ limit one considers $N \rightarrow \infty$ fields with an $O(N)$ invariant self-coupling $g / N$. Since grand unified theories contain a large number of scalar fields, the large $N$ limit seems a more realistic effective model than the Hartree approximation for the inflationary universe. Particle production during preheating turns to be much more effective in the large $N$ limit than within the Hartree approximation (see sec. VII and [14]). In addition, the field evolution is definitely model dependent. For example, in the fine-tuned two-fields model (such that the Mathieu equation applies) studied in ref. [54], parametric resonance in Hartree approximation is significative only for a limited range of parameters.

We have considered in these Lectures single-mass inflaton models. The Hartree approximation for a two-field model (with unequal masses) is presented in the second reference under [10]. In such cases, one must carefully apply the Hartree approximation to all fields in the model self-consistently. Applying Hartree to one field and just doing perturbation theory in the other field leads to inconsistencies as noticed in ref. [8].

It can be argued that the inflationary paradigm as currently understood is one of the greatest applications of quantum field theory. The imprint of quantum mechanics is everywhere, from the dynamics of the inflaton, to the generation of metric perturbations, through to the reheating of the universe. It is clear then that we need to understand the quantum mechanics of inflation in as deep a manner as possible so as to be able to understand what we are actually testing via the CMBR temperature anisotropies, say.

What we have found in our work is that the quantum mechanics of inflation is extremely subtle. We now understand that it involves both non-equilibrium as well as non-perturbative dynamics and that what you start from may not be what you wind up with at the end!

In particular, we see now that the correct interpretation of the non-perturbative growth of quantum fluctuations via spinodal decomposition is that the background zero mode must be redefined through the process of zero mode reassembly that we have discovered. When this is done (and only when!) we can interpret inflation in terms of the usual slow-roll 
approach with the now small quantum fluctuations around the redefined zero mode driving the generation of metric perturbations.

We have studied the non-equilibrium dynamics of a 'new inflation' scenario in a selfconsistent, non-perturbative framework based on a large $N$ expansion, including the dynamics of the scale factor and backreaction of quantum fluctuations. Quantum fluctuations associated with superhorizon modes grow exponentially as a result of the spinodal instabilities and contribute to the energy momentum tensor in such a way as to end inflation consistently.

Analytical and numerical estimates have been provided that establish the regime of validity of the classical approach. We find that these superhorizon modes re-assemble into an effective zero mode and unambiguously identify the composite field that can be used as an effective expectation value of the inflaton field whose classical dynamics drives the evolution of the scale factor. This identification also provides the initial condition for this effective zero mode.

A consistent criterion is provided to extract small fluctuations that will contribute to cosmological perturbations from large non-perturbative spinodal fluctuations. This is an important ingredient for a consistent calculation and interpretation of cosmological perturbations. This criterion requires that the model must provide many more than 60 e-folds to identify the 'small perturbations' that give rise to scalar metric (curvature) perturbations. We then use this criterion combined with the gauge invariant approach to obtain the dynamics of the Bardeen variable and the spectrum for scalar perturbations.

We find that during the inflationary epoch, superhorizon modes of the Bardeen potential grow exponentially in time reflecting the spinodal instabilities. These long-wavelength instabilities are manifest in the spectrum of scalar density perturbations and result in an index that is less than one, i.e. a 'red' power spectrum, providing more power at long wavelength. We argue that this red spectrum is a robust feature of potentials that lead to spinodal instabilities in the region in field space associated with inflation and can be interpreted as an imprint of the phase transition on the cosmological background. Tensor perturbations on the other hand, are not modified by these features, they have much smaller amplitude and a Harrison-Zeldovich spectrum.

We made contact with the reconstruction program and validated the results for these type of models based on the slow-roll assumption, despite the fact that our study does not involve such an approximation and is non-perturbative.

Finally we have studied the quantum to classical crossover and decoherence of quantum fluctuations by studying the full evolution of the density matrix, thus making contact with the concept of 'decoherence without decoherence' [43] which is generalized to the interacting case. In the case under consideration decoherence and classicalization is a consequence of spinodal growth of superhorizon modes and the presence of a horizon. The phases of the mode functions freeze out and the amplitudes of the superhorizon modes grow exponentially during the inflationary stage, again as a result of long-wavelength instabilities. As a result field configurations with large amplitudes have non-vanishing probabilities to be represented in the dynamical density matrix. In the situation considered, the quantum to classical crossover of cosmological perturbations is directly related to the classicalization of superhorizon matter field modes that grow exponentially upon horizon crossing during inflation. The diagonal elements of the density matrix in the Schroedinger representation 
can be interpreted as a classical distribution function, whereas the off-diagonal elements are strongly suppressed during inflation.

\section{ACKNOWLEDGEMENTS:}

The authors thank J. Baacke, K. Heitman, L. Grishchuk, A. Singh and M. Srednicki, E. Weinberg, E. Kolb, A. Dolgov and D. Polarski, for conversations and discussions and especially to C. Destri in collaboration with whom sec. II was written. D. B. thanks the N.S.F for partial support through the grant awards: PHY-9605186 and INT-9216755, the Pittsburgh Supercomputer Center for grant award No: PHY950011P and LPTHE for warm

hospitality. R. H., D. C. and S. P. K. were supported by DOE grant DE-FG02-91-ER40682. H. J. de V. acknowledges partial support by NATO. 


\section{REFERENCES}

[1] A. H. Guth, Phys. Rev. D23, 347 (1981).

[2] For thorough reviews of standard and inflationary cosmology see: E. W. Kolb and M. S. Turner, The Early Universe (Addison Wesley, Redwood City, C.A. 1990). A. Linde, Particle Physics and Inflationary Cosmology, (Harwood 1990) and ref. [5].

[3] For more recent reviews see: M. S. Turner, astro-ph-9703197; astro-ph-9703196; astroph-9703174; astro-ph-9703161; astro-ph-9704062; astro-ph-9704024. A. Linde, in Current Topics in Astrofundamental Physics, Proceedings of the Chalonge Erice School, N. Sánchez and A. Zichichi Editors, Nato ASI series C, vol. 467, 1995, Kluwer Acad. Publ. A. R. Liddle, astro-ph-9612093, Lectures at the Casablanca School Morocco, 1996.

[4] G. Smoot, in the Proceedings of the Vth. Erice Chalonge School on Astrofundamental Physics, p. 407-484, N. Sánchez and A. Zichichi eds., World Scientific, 1997.

A. R. Liddle and D. H. Lyth, Phys. Rep. 231, 1 (1993).

[5] for reviews of inflation, see R. Brandenberger, Rev. of Mod. Phys. 57, 1 (1985); Int. J. Mod. Phys. A2, 77 (1987) and ref. [2].

[6] A. D. Dolgov and A. D. Linde, Phys. Lett. B116, 329 (1982);

L. F. Abbott, E. Farhi and M. Wise, Phys. Lett. B117, 29 (1982).

[7] J. Traschen and R. Brandenberger, Phys Rev D42, 2491 (1990);

Y. Shtanov, J. Traschen and R. Brandenberger, Phys. Rev. D51, 5438 (1995).

[8] L. Kofman, A. Linde and A. Starobinsky, Phys. Rev. Lett. 73, 3195 (1994) and 76, 1011 (1996); Phys. Rev. D56, 3258 (1997); gr-qc/9508019 (1995). L. Kofman, astroph/9605155 (1996).

[9] D. Boyanovsky and H. J. de Vega, Phys. Rev. D47, 2343 (1993).

[10] D. Boyanovsky, H. J. de Vega, R. Holman, D.-S. Lee and A. Singh, Phys. Rev. D51, 4419 (1995);

D. Boyanovsky, M. D’Attanasio, H. J. de Vega, R. Holman and D. S. Lee, Phys. Rev. D52, 6805 (1995);

For reviews see, D. Boyanovsky, H. J. de Vega and R. Holman, in the Proceedings of the Second Paris Cosmology Colloquium, Observatoire de Paris, June 1994, p. 127215, H. J. de Vega and N. Sánchez Editors, World Scientific, 1995; D. Boyanovsky, M. D'Attanasio, H. J. de Vega, R. Holman and D.-S. Lee, 'New aspects of reheating', in the Proceedings of the Erice Chalonge School, 'String Gravity and Physics at the Planck Energy Scale', NATO ASI, N. Sánchez and A. Zichichi Editors, Kluwer 1996, p. 451-492.

[11] D. Boyanovsky, H.J. de Vega, R. Holman, J.F.J. Salgado, Phys. Rev. D54, 7570 (1996). D. Boyanovsky, H. J. de Vega and R. Holman in the Proceedings of the Vth. Erice Chalonge School on Astrofundamental Physics, p. 183-270, N. Sánchez and A. Zichichi eds., World Scientific, 1997.

[12] D. Boyanovsky, C. Destri, H.J. de Vega, R. Holman and J.F.J. Salgado, 'Asymptotic Dynamics in Scalar Field Theory: Anomalous Relaxation', hep-ph/9711384.

[13] D. Boyanovsky, H. J. de Vega, and R. Holman, Phys. Rev. D49, 2769 (1994).

[14] D. Boyanovsky, D. Cormier, H. J. de Vega, R. Holman, A. Singh, M. Srednicki, Phys. Rev. D56, 1939 (1997).

[15] D. Boyanovsky, D. Cormier, H. J. de Vega and R. Holman, Phys. Rev. D55, 3373 (1997).

[16] 'Non-Perturbative Quantum Dynamics of a New Inflation Model', D. Boyanovsky, D. 
Cormier, H. J. de Vega, R. Holman and S. P. Kumar, hep-ph/9709232 to appear in Phys. Rev. D, 15 february 1998.

[17] D. Boyanovsky, H.J. de Vega, R. Holman and J. F. J. Salgado, astro-ph/9609007, to appear in the Proceedings of the Paris Euronetwork Meeting 'String Gravity'.

[18] D. Boyanovsky, D-S. Lee, and A. Singh, Phys. Rev. D48, 800 (1993).

[19] D. Boyanovsky, M. D'Attanasio, H. J. de Vega and R. Holman, Phys. Rev. D54, 1748 (1996), and references therein.

[20] H.J. de Vega and J. F. J. Salgado, Phys. Rev. D 56, 6524 (1997).

[21] S. Yu. Khlebnikov and I.I. Tkachev, Phys. Rev. Lett. 77, 219 (1996); S. Yu. Khlebnikov and I.I. Tkachev, hep-ph/9608458 (1996).

[22] D.T. Son, Phys. Rev. D54, 3745 (1996); hep-ph/9601377.

[23] L. Kofman, A. Linde and A. Starobinsky, Phys. Rev. Lett. 76, 1011, (1996); I.I Tkachev, Phys. Lett. B376, 35 (1996); A. Riotto and I.I. Tkachev, Phys. Lett. B385, 57 (1996); E.W. Kolb and A. Riotto, astro-ph/9602095 (1996).

[24] D.I. Kaiser, Phys. Rev D53, 1776 (1996) and D56, 706 (1997), hep-ph/9707516.

[25] H. Fujisaki, K. Kumekawa, M. Yamaguchi and M.Yoshimura, Phys. Rev. D53, 6805 (1996); M. Yoshimura, Progr. Theor. Phys. 94, 873 (1995); hep-ph/9605246.

[26] J. Schwinger, J. Math. Phys. 2, 407 (1961); P. M. Bakshi and K. T. Mahanthappa, J. Math. Phys. 4, 1 (1963); ibid, 12; L. V. Keldysh, Sov. Phys. JETP 20, 1018 (1965); A. Niemi and G. Semenoff, Ann. of Phys. (N.Y.) 152, 105 (1984); Nucl. Phys. B [FS10], 181 (1984); E. Calzetta, Ann. of Phys. (N.Y.) 190, 32 (1989); R. D. Jordan, Phys. Rev. D33, 444 (1986); N. P. Landsman and C. G. van Weert, Phys. Rep. 145, 141 (1987); R. L. Kobes and K. L. Kowalski, Phys. Rev. D34, 513 (1986); R. L. Kobes, G. W. Semenoff and N. Weiss, Z. Phys. C29, 371 (1985).

[27] For a thorough exposition of non-equilibrium methods in cosmology see, for example: E. Calzetta and B.-L. Hu, Phys. Rev. D35, 495 (1988); ibid D37, 2838 (1988); J. P. Paz, Phys. Rev. D41, 1054 (1990); ibid D42, 529 (1990); B.-L. Hu in Bannf/Cap Workshop on Thermal Field Theories: proceedings, edited by F.C. Khanna, R. Kobes, G. Kunstatter, H. Umezawa (World Scientific, Singapore, 1994), p.309 and in the Proceedings of the Second Paris Cosmology Colloquium, Observatoire de Paris, edited by H. J. de Vega and N. Sánchez (World Scientific, Singapore, 1995), p.111 and references therein.

[28] H. Leutwyler and S. Mallik, Ann. of Phys. (N.Y.) 205, 1 (1991).

[29] O. Eboli, R. Jackiw and S.-Y. Pi, Phys. Rev. D37, 3557 (1988);

M.Samiullah, O. Eboli and S-Y. Pi, Phys. Rev. D44, 2335 (1991).

J. Guven, B. Liebermann and C. Hill, Phys. Rev. D39, 438 (1989).

[30] N. D. Birrell and P.C.W. Davies, Quantum fields in curved space (Cambridge Univ. Press, Cambridge, 1986).

[31] J. Baacke, K. Heitmann and C. Pätzold, Phys. Rev. D55, 2320 (1997), hep-ph/9706274, hep-th/9711144 and hep-ph/9712506.

[32] M. Abramowitz and I.E. Stegun (eds.), Handbook of Mathematical Functions (National Bureau of Standards, Washington, D.C., 1972), chapter 13.

[33] 'The Scalar, Vector and Tensor Contributions to CMB anisotropies from Topological Defects', by N. Turok, Ue-Li Pen, U. Seljak astro-ph/9706250 (1997). 'The case against scaling defect models of cosmic structure formation', A. Albrecht, R. A. Battye, J. 
Robinson, astro-ph/9707129 (1997). 'CMB Anisotropy Induced by Cosmic Strings on Angular Scales > 15", by B. Allen, R. R. Caldwell, S. Dodelson, L. Knox, E. P. S. Shellard, A. Stebbins, astro-ph/9704160 (1997).

[34] D. H. Lyth, hep-ph-9609431 (1996). S. Dodelson, W. H. Kinney and E. W. Kolb, astroph-9702166.

[35] A.D. Linde, Phys. Lett. B116, 335 (1982). A. Vilenkin and L. H. Ford, Phys. Rev. D26, 1231 (1982). A. Vilenkin, Nucl. Phys. B226, 504 (1983); Nucl. Phys. B226, 527 (1986).

[36] A. Vilenkin, Phys. Lett. B115, 91 (1982).

[37] P. J. Steinhardt and M. S. Turner, Phys. Rev. D29, 2162, (1984).

[38] A. Guth and S-Y. Pi, Phys. Rev. D32, 1899 (1985).

[39] For non-equilibrium methods in different contexts see for example: F. Cooper, J. M . Eisenberg, Y. Kluger, E. Mottola, B. Svetitsky, Phys. Rev. Lett. 67, 2427 (1991); F. Cooper, J. M. Eisenberg, Y, Kluger, E. Mottola, B. Svetitsky, Phys. Rev. D48, 190 (1993).

[40] F. Cooper and E. Mottola, Mod. Phys. Lett. A 2, 635 (1987); F. Cooper, S. Habib, Y. Kluger, E. Mottola, J. P. Paz, P. R. Anderson, Phys. Rev. D50, 2848 (1994). F. Cooper, S.-Y. Pi and P. N. Stancioff, Phys. Rev. D34, 3831 (1986). F. Cooper and E. Mottola, Phys. Rev. D36, 3114 (1987).

F. Cooper, Y. Kluger, E. Mottola, J. P. Paz, Phys. Rev. D51, 2377 (1995).

[41] S. A. Ramsey, B. L. Hu, Phys. Rev. D56, 678 (1997).

[42] D. Boyanovsky, H. J. de Vega and R. Holman, Phys. Rev. D 51, 734 (1995).

D. Boyanovsky, H. J. de Vega, R. Holman and S. Prem Kumar,

Phys. Rev. D56, 3929 and 5233 (1997).

The last reference under [40].

[43] D. Polarski and A. A. Starobinsky, Class. Quant. Grav. 13, 377 (1996);

J. Lesgourgues, D. Polarski and A. A. Starobinsky, Nucl. Phys. B497, 479 (1997).

[44] L. P. Grishchuk, Phys. Rev. D 45, 4717 (1992).

[45] V. F. Mukhanov, H. A. Feldman and R. H. Brandenberger, Phys. Rep. 215, 293 (1992).

[46] J. Bardeen, Phys. Rev. D22, 1882 (1980).

[47] R. R. Caldwell, Class. Quant. Grav. 13, 2437 (1995).

J. Martin and D. J. Schwarz, gr-qc-9704049 (1997).

[48] K. M. Gorski, A. J. Banday, C. L. Bennett, G. Hinshaw, A. Kogut and G. F. Smoot, (astro-ph-9601063) (submitted to ApJ. Lett.).

[49] L. P. Grishchuk, Phys. Rev. D. 52, 5549, (1995); Proceedings of the Erice Chalonge School NATO ASI on 'String Gravity and Physics at the Planck Scale', Ed. N. Sánchez and A. Zichichi, (Kluwer, 1996), p. 369; Phys. Rev. D53 (1996) 6784; Proceedings of the NATO ASI on 'Current Topics in Astrofundamental Physics', Ed. N. Sanchez and A. Zichichi (Kluwer, 1995), p. 205.

[50] J. E. Lidsey, A. R. Liddle, E. W. Kolb, E. J. Copeland, T. Barreiro, M. Abney, Rev. Mod. Phys. 69, 373, (1997).

[51] E. D. Stewart and D. H. Lyth, Phys. Lett. B302, 171 (1993).

[52] S. Habib, Phys. Rev. D 46, 2408 (1992); Phys. Rev. D 42, 2566, (1990);

S. Habib and R. Laflamme, Phys. Rev. D42, 4056, (1990), and references therein.

[53] See eq.(53) in the first reference under [43].

[54] I. Zlatev, G. Huey and P. J. Steinhardt, astro-ph/9709006. 